\title{
Otto Vejvoda
}

On perturbed nonlinear boundary value problems

Czechoslovak Mathematical Journal, Vol. 11 (1961), No. 3, 323-364

Persistent URL: http://dml.cz/dmlcz/100464

\section{Terms of use:}

(C) Institute of Mathematics AS CR, 1961

Institute of Mathematics of the Czech Academy of Sciences provides access to digitized documents strictly for personal use. Each copy of any part of this document must contain these Terms of use.

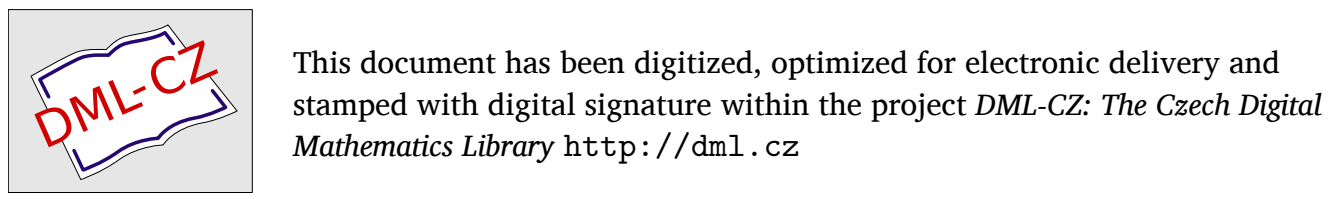




\title{
ON PERTURBED NONLINEAR BOUNDARY VALUE PROBLEMS
}

\author{
O. Vejvoda, Praha \\ (Received February 5, 1960 - in revised form February 10, 1961)
}

\begin{abstract}
The existence of a solution of a boundary value problem $(0.1)$ is investigated under the assumption that we know how to solve the shortened boundary value problem $(0.2)$.

In particular the autonomous case (i. e. $f$ and $\mathbf{g}$ do not depend on $t$ explicitly) is studied thoroughly and a class of these autonomous boundary value problems which show the same "anomalies" as problems with periodic boundary conditions is singled out.
\end{abstract}

In this paper I prove some theorems on the existence of a solution of a nonlinear boundary value problem

$$
\dot{\mathbf{x}}=\mathbf{f}(t, \mathbf{x})+\varepsilon \mathbf{g}(t, \mathbf{x}, \varepsilon), \quad \mathbf{u}(\mathbf{x}(a), \mathbf{x}(b))+\varepsilon \mathbf{v}(\mathbf{x}(a), \mathbf{x}(b), \varepsilon)=0
$$

where $\mathbf{x}, f, \mathbf{g}, \boldsymbol{u}$ and $\mathbf{v}$ are $n$-vectors, under the assumption that the existence of a solution of some other more simple ("shortened") boundary value problem

$$
\dot{\boldsymbol{y}}=\mathbf{f}(t, \mathbf{y}), \quad \mathbf{u}(\mathbf{y}(a), \mathbf{y}(b))=0
$$

is guaranteed. The shortened boundary value problem is got by equating to zero a "small" parameter $\varepsilon$. Then only those solutions of the original boundary value problem are sought for which tend to some solution of the shortened problem as $\varepsilon \rightarrow 0$. This method has been used until now as far as I know in problems with periodic boundary conditions only (especially when the shortened system of differential equations was linear - so called quasi-linear systems) [1], [2], [3], [4].

To prove the theorems the variation-of-constants method for nonlinear systems and implicit function theorem are used as principal tools. Necessary theorems are listed in sec. 2.

In sec. 1 some theorems on linear boundary value problems which are needed later are listed for the reader's convenience. (Maybe only remarks 1.1 and 1.2 are original.) See for instance [1], [5].

I wish to express my gratitude to DSc J. KURZWEIL whose advices contributed considerably to the improvement of this paper. 
Some notations and definitions. In this paper all given numbers, functions, matrices etc. are assumed real without saying so explicitly.

If not stated otherwise a $n$-vector means always a column $n$-dimensional vector. Given a $m \times n$ matrix $\boldsymbol{C}, \boldsymbol{C}^{\prime}$ will denote the transposed matrix.

The inner product of two $n$-vectors $\boldsymbol{u}$ and $\mathbf{v}$ is defined as

$$
\mathbf{u} \cdot \mathbf{v}=\sum_{j=1}^{n} u_{j} v_{j}=\mathbf{u}^{\prime} \mathbf{v}=\mathbf{v}^{\prime} \mathbf{u} .
$$

Given two $m \times n$ matrices $\boldsymbol{A}=\left(a_{j k}\right), \boldsymbol{B}=\left(b_{j k}\right), \boldsymbol{A}: \mathbf{B}$ denotes the $m \times 2 n$ matrix

$$
\boldsymbol{A}: \mathbf{B}=\left(\begin{array}{cccccc}
a_{11} & \ldots & a_{1 n} & b_{11} & \ldots & b_{1 n} \\
\ldots & \ldots & \ldots & \ldots & \ldots & \ldots \\
a_{m 1} & \ldots & a_{m n} & b_{m 1} & \ldots & b_{m n}
\end{array}\right) .
$$

The rank of a matrix $\boldsymbol{C}$ will be denoted by $\chi(\boldsymbol{C})$.

$\boldsymbol{E}_{n}$ will denote the $n \times n$ unit matrix. If no confusion can arise the index $n$ will be omitted.

Everywhere in the sequel $\mathscr{N}$ denotes the naturally ordered set $\{1,2, \ldots, n\}$. If $\mathscr{S}$ is a (naturally) ordered subset of $\mathscr{N}$, then $\overline{\mathscr{S}}$ denotes the (naturally) ordered complement of $\mathscr{S}$ with respect to $\mathscr{N}$. (All subsets of $\mathscr{N}$ will be supposed naturally ordered.) The number of elements of a set $\mathscr{A} \subset \mathscr{N}$ will be denoted by $v(\mathscr{A})$.

Let $C$ be a $n \times n$ matrix and let $\mathscr{L} \subset \mathscr{N}, \mathscr{M} \subset \mathcal{N}$. Then let the notation be introduced

$$
\boldsymbol{C}_{\mathscr{L} \mathscr{M}}=\left(c_{p q}\right), \quad p \in \mathscr{L}, \quad q \in \mathscr{M} .
$$

If $\mathscr{M}=\mathscr{N}$, we shall write shortly $\boldsymbol{C}_{\mathscr{L}}$ instead of $\boldsymbol{C}_{\mathscr{L} \mathscr{N}}$.

Similarly, if $\mathbf{x}$ is a $n$-vector and $\mathscr{L} \subset \mathcal{N}$, then

$$
\mathbf{x}_{\mathscr{L}}=\left(x_{j}\right), \quad j \in \mathscr{L} .
$$

The signs $\dot{+}$ and - are defined by

$$
\mathbf{x}=\mathbf{x}_{\mathscr{L}}+\mathbf{x}_{\overline{\mathscr{L}}}, \quad \mathbf{x}_{\mathscr{L}}=\mathbf{x} \dot{-x_{\overline{\mathscr{L}}}} .
$$

Let $E^{n}$ denote a $n$-dimensional Euclidian space.

A closed (finite) interval $a \leqq t \leqq b$ will be denoted by $\langle a, b\rangle$, an open interval $a<t<b$ will be denoted by $(a, b)$, a half-open interval $a<t \leqq b$ will be denoted by $(a, b>$ and analogously in other cases.

The notation $E[\mathbf{x} \mid V(\mathbf{x})]$ will be used to denote the set of all points $\mathbf{x}$ having the property $V(\mathbf{x})$.

By a neighborhood $\mathfrak{N}\left(\mathrm{c}^{*}, \delta\right)$ of a point $\mathrm{c}^{*} \in E^{n}$ we shall mean the open sphere $\mathfrak{N}\left(\mathrm{c}^{*}, \delta\right)=E\left[\mathrm{c} \mid\left\|\mathrm{c}-\mathrm{c}^{*}\right\|<\delta\right], \delta>0$.

Point sets $\mathfrak{X}$ and $\mathfrak{Y}$ being given, $\mathfrak{X} \times \mathfrak{Y}$ will denote the Cartesian product of these sets.

Let $\boldsymbol{f}(\boldsymbol{x}, \boldsymbol{y}, \boldsymbol{z})$ be a vector-function of vectors $\boldsymbol{x}, \boldsymbol{y}, \mathbf{z}$ defined for $\mathbf{x} \in \mathfrak{X}, \boldsymbol{y} \in \mathfrak{Y}, \mathbf{z} \in \mathcal{Z}$, where $\mathfrak{X}, \mathfrak{Y}, \mathfrak{Z}$ are open sets. We say that $\boldsymbol{f}(\boldsymbol{x}, \boldsymbol{y}, \mathbf{z})$ is of class $C^{p, q, \boldsymbol{r}}$ on $\mathfrak{X} \times \mathfrak{Y} \times \mathfrak{Z}$ (and write $\boldsymbol{f}(\mathbf{x}, \mathbf{y}, \mathbf{z}) \in C^{p, q, r}$ ) if it has continuous partial derivatives of order $p$ or $q$ or $r$ 
with respect to all components of the vector $\mathbf{x}$ or $\mathbf{y}$ or $\mathbf{z}$, respectively, for $(\mathbf{x}, \mathbf{y}, \mathbf{z}) \in$ $\in \mathfrak{X} \times \mathfrak{Y} \times \mathfrak{Z}$. If the highest derivatives of order $p$ with respect to $\mathbf{x}$ fulfil a Lipschitz condition with respect to the vector $x$, then we write $f(x, y, z) \in C^{p L, q, r}$ and analogously in other cases.

If the vector-function $\boldsymbol{f}(t, \mathbf{x})$ is defined for $t \in \mathfrak{T}, \mathbf{x} \in \mathfrak{X}$, where $\mathfrak{I}$ is a finite closed interval $\langle a, b\rangle \in E^{1}$, then by saying that $\boldsymbol{f}$ is of class $C^{1}$ in $t$ for $(t, \mathbf{x}) \in \mathfrak{Z} \times \mathfrak{X}$ we mean that it is of class $C^{1}$ in int $\mathfrak{I}$ and has a right-hand derivative at $a$ and a left-hand derivative at $b$ with respect to $t$ for all $\mathbf{x} \in \mathfrak{X}$.

Let $\boldsymbol{f}(\mathbf{x})$ be a $m$-dimensional vector-function of an $n$-vector $\mathbf{x}$ of class $C^{1}$ on an open set $\mathfrak{X} \subset E^{n}$. Then

$$
\frac{\mathrm{D} f}{\mathbf{D} \mathbf{x}}(\mathbf{x})=f_{\mathbf{x}}(\mathbf{x})=\left(\frac{\partial f_{j}}{\partial x_{k}}\right) \quad(j=1,2, \ldots, m, k=1 ; 2, \ldots, n)
$$

will denote the $m \times n$ functional matrix of $\mathbf{f}(\mathbf{x})$.

\section{LINEAR BOUNDARY VALUE PROBLEMS}

Let us consider the system

$$
I(\mathbf{x}) \equiv \dot{\boldsymbol{x}}+\mathbf{A}(t) \mathbf{x}=0 \quad\left(\dot{\boldsymbol{x}}=\frac{\mathrm{d} \mathbf{x}}{\mathrm{d} t}\right)
$$

where $\mathbf{x}$ is an $n$-vector and $\mathbf{A}(t)$ is an $n \times n$ matrix of class $C^{0}$ on a finite closed interval $\langle a, b\rangle$.

Let $\mathbf{u}(\mathbf{x}(a), \mathbf{x}(b))$ be an $m$-vector of $m$ boundary value forms defined by

$$
\mathbf{u}(\mathbf{x}(a), \mathbf{x}(b)) \equiv \mathbf{M} \mathbf{x}(a)+\mathbf{N} \mathbf{x}(b),
$$

where $\boldsymbol{M}$ and $\boldsymbol{N}$ are two $m \times n$ constant matrices $(m<2 n)$.

If $\chi(\mathbf{M}: \mathbf{N})=m$, we say that boundary forms have the rank $m$.

Then

$$
\mathbf{u}\left(\mathbf{x}_{a}, \mathbf{x}_{b}\right) \equiv \mathbf{M} \mathbf{x}(a)+\mathbf{N} \mathbf{x}(b)=0
$$

represents $m$ boundary conditions of rank $m$. (For the sake of brevity we shall write $\boldsymbol{x}_{a}, \boldsymbol{x}_{b}$ instead of $\mathbf{x}(a), \mathbf{x}(b)$, respectively.)

The system

$$
I^{*}(\mathbf{x}) \equiv-\mathbf{x}+\boldsymbol{A}^{\prime}(t) \mathbf{x}=0
$$

(which is evidently equivalent with the system

$$
I^{*}(\mathbf{x}) \equiv-\dot{x}^{\prime}+\mathbf{x}^{\prime} \mathbf{A}(t)=0,
$$

is called the adjoint system to (1.1).

Let $\tilde{\boldsymbol{M}}$ and $\tilde{\boldsymbol{N}}$ be constant $(2 n-m) \times n$ matrices. Let the matrix

$$
\left(\begin{array}{cc}
\mathbf{M} & \mathbf{N} \\
\tilde{\mathbf{M}} & \tilde{\mathbf{N}}
\end{array}\right)
$$


be of rank $2 n$. Then boundary conditions

$$
\overline{\mathbf{u}}\left(\boldsymbol{x}_{\boldsymbol{a}}, \mathbf{x}_{\boldsymbol{b}}\right) \equiv \tilde{\mathbf{M}} \mathbf{x}(a)+\tilde{\mathbf{N}} \mathbf{x}(b)=0
$$

are called complementary boundary conditions to (1.2) and $\tilde{u}\left(\boldsymbol{x}_{a}, \boldsymbol{x}_{b}\right)$ are called complementary boundary forms to $\mathbf{u}\left(\boldsymbol{x}_{a}, \boldsymbol{x}_{b}\right)$.

Theorem 1.1. (Green's formula.) Let $\mathbf{u}(t)$ and $\mathbf{v}(t)$ be n-vectors of class $C^{1}$ on $\langle a, b\rangle$. Let $a \leqq t_{1}<t_{2} \leqq b$. Then

$$
\int_{t_{1}}^{t_{2}}\left[I(\mathbf{u}) \cdot \mathbf{v}-\mathbf{u} \cdot I^{*}(\mathbf{v})\right] \mathrm{d} t=\mathbf{u}\left(t_{2}\right) \cdot \mathbf{v}\left(t_{2}\right)-\mathbf{u}\left(t_{1}\right) \cdot \mathbf{v}\left(t_{1}\right) .
$$

Theorem 1.2. (Boundary-form formula.) Let boundary forms $\mathbf{u}\left(\mathbf{x}_{a}, \mathbf{x}_{b}\right)$ defined by $m \times n$ matrices $\boldsymbol{M}$ and $\mathbf{N}$ be of rank $m$ and let $\tilde{\mathbf{u}}\left(\mathbf{x}_{a}, \mathbf{x}_{b}\right)$ be any complementary boundary forms to $\mathbf{u}\left(\mathbf{x}_{a}, \boldsymbol{x}_{b}\right)$.

Then there exist unique boundary forms $\tilde{\mathbf{u}}^{*}\left(\boldsymbol{x}_{a}, \boldsymbol{x}_{b}\right)$ and $\mathbf{u}^{*}\left(\boldsymbol{x}_{a}, \boldsymbol{x}_{b}\right)$ of ranks $m$ and $2 n-m$, respectively, such that for arbitrary n-vectors $\mathbf{x}_{a}, \boldsymbol{x}_{b}, \mathbf{y}_{a}$ and $\mathbf{y}_{b}$

$$
\mathbf{x}_{b} \cdot \boldsymbol{y}_{b}-\boldsymbol{x}_{\boldsymbol{a}} \cdot \mathbf{y}_{\boldsymbol{a}}=\boldsymbol{u}\left(\boldsymbol{x}_{a}, \mathbf{x}_{b}\right) \cdot \tilde{\mathbf{u}}^{*}\left(\boldsymbol{y}_{a}, \boldsymbol{y}_{b}\right)+\tilde{\mathbf{u}}\left(\boldsymbol{x}_{a}, \boldsymbol{x}_{b}\right) \cdot \mathbf{u}^{*}\left(\boldsymbol{y}_{a}, \mathbf{y}_{b}\right) .
$$

(Forms $\tilde{\mathbf{u}}^{*}$ and $\boldsymbol{u}^{*}$ are called adjoint to forms $\tilde{\mathbf{u}}$ and $\boldsymbol{u}$, respectively.)

If $\tilde{\mathbf{u}}_{1}$ is another system of complementary forms to $\mathbf{u}$, and $\tilde{\mathbf{u}}_{1}^{*}$ and $\mathbf{u}_{1}^{*}$ are corresponding adjoint forms, then

$$
\begin{aligned}
& \mathbf{u}_{1}^{*}=K \mathbf{u}^{*}, \\
& \tilde{\mathbf{u}}_{1}^{*}=\tilde{\mathbf{u}}^{*}+L \mathbf{u}^{*},
\end{aligned}
$$

where $\boldsymbol{K}$ is a regular $(2 n-m) \times(2 n-m)$ matrix and $\boldsymbol{L}$ is an $m \times m$ matrix.

The relation (1.5) is known. The relation (1.6) can easily be proved with help of assertion (a) of the following

Lemma 1.1. Let $\boldsymbol{f}$ and $\mathbf{g}$ be $k$-vectors. Let $\boldsymbol{F}$ be a regular $k \times k$ matrix such that the first $j(1 \leqq j<k)$ components of the vector $\tilde{\boldsymbol{f}}=\mathbf{F f}$ are identical with the first $j$ components of $\boldsymbol{f}$. Then there exists a unique regular $k \times k$ matrix $\boldsymbol{G}$ such that if $\tilde{\mathbf{g}}=\mathbf{G g}$ then $\tilde{\boldsymbol{f}} \cdot \tilde{\mathbf{g}}=\mathbf{f} . \mathbf{g}$ and has the following properties:

(a) the first $j$ components of $\tilde{\mathbf{g}}$ are equal to the first $j$ components of $\mathbf{g}$ to which linear combinations of the last $k-j$ components are added;

(b) the last $k-j$ components of $\tilde{\mathbf{g}}$ are linear combinations of the last $k-j$ components of $\mathbf{g}$ with a regular coefficient matrix.

Remark 1.1. $(2 n-m) \times n$ matrices $\boldsymbol{P}, \mathbf{Q}$ and $m \times n$ matrices $\tilde{\boldsymbol{P}}, \tilde{\mathbf{Q}}$ defining adjoint boundary forms $\boldsymbol{u}^{*}$ and $\tilde{\boldsymbol{u}}^{*}$, respectively, may easily be determined in an explicit form.

Substituting the expressions for $\boldsymbol{u}, \tilde{\mathbf{u}}, \tilde{\boldsymbol{u}}^{*}$ and $\boldsymbol{u}^{*}$ into (1.4) we see that (1.4) is fulfilled for arbitrary $\boldsymbol{x}_{a}, \boldsymbol{x}_{b}, \boldsymbol{y}_{a}$ and $\boldsymbol{y}_{b}$ if and only if

$$
\begin{array}{ll}
\tilde{\mathbf{P}}^{\prime} \mathbf{M}+\mathbf{P}^{\prime} \tilde{\mathbf{M}}=-\mathbf{E}, & \tilde{\mathbf{Q}}^{\prime} \mathbf{M}+\mathbf{Q}^{\prime} \tilde{\mathbf{M}}=0, \\
\tilde{\mathbf{P}}^{\prime} \mathbf{N}+\mathbf{P}^{\prime} \tilde{\mathbf{N}}=0, & \tilde{\mathbf{Q}}^{\prime} \mathbf{N}+\mathbf{Q}^{\prime} \tilde{\mathbf{N}}=\mathbf{E} .
\end{array}
$$


Solving these two systems and writing

$$
\left(\begin{array}{ll}
\mathbf{M} & \boldsymbol{N} \\
\tilde{M} & \tilde{N}
\end{array}\right)^{-1}=\left(\begin{array}{cc}
\tilde{R} & R \\
\tilde{S} & S
\end{array}\right)
$$

where $\tilde{\boldsymbol{R}}$ and $\tilde{\boldsymbol{S}}$ are $n \times m$ matrices and $\boldsymbol{R}$ and $\boldsymbol{S}$ are $n \times(2 n-m)$ matrices, we get

$$
\begin{array}{ll}
\tilde{\mathbf{P}}^{\prime}=-\tilde{\mathbf{R}}, & \mathbf{P}^{\prime}=-\boldsymbol{R}, \\
\tilde{\mathbf{Q}}^{\prime}=\tilde{S}, & \mathbf{Q}^{\prime}=\mathbf{S} .
\end{array}
$$

In virtue of

it follows

$$
\left(\begin{array}{ll}
\mathbf{M} & \boldsymbol{N} \\
\tilde{M} & \tilde{\mathbf{N}}
\end{array}\right)\left(\begin{array}{ll}
\tilde{R} & \boldsymbol{R} \\
\tilde{\boldsymbol{S}} & \boldsymbol{S}
\end{array}\right)=\left(\begin{array}{cc}
\mathbf{M} & \boldsymbol{N} \\
\tilde{M} & \tilde{\mathbf{N}}
\end{array}\right)\left(\begin{array}{cc}
-\tilde{\boldsymbol{P}}^{\prime} & -\boldsymbol{P}^{\prime} \\
\tilde{\mathbf{Q}}^{\prime} & \mathbf{Q}^{\prime}
\end{array}\right)=\left(\begin{array}{cc}
E_{m} & 0 \\
0 & E_{2 n-m}
\end{array}\right)
$$

$$
\begin{aligned}
& -\mathbf{M} \tilde{\mathbf{P}}^{\prime}+\mathbf{N} \tilde{Q}^{\prime}=E_{m}, \quad-M \mathbf{P}^{\prime}+\mathbf{N} \mathbf{Q}^{\prime}=0, \\
& -\tilde{\mathbf{M}} \tilde{\mathbf{P}}^{\prime}+\tilde{\mathbf{N}} \tilde{\mathbf{Q}}^{\prime}=0, \quad-\tilde{M} \mathbf{P}^{\prime}+\tilde{\mathbf{N}} \mathbf{Q}^{\prime}=E_{2 n-m} .
\end{aligned}
$$

Let $\boldsymbol{u}\left(\boldsymbol{x}_{\boldsymbol{a}}, \boldsymbol{x}_{\boldsymbol{b}}\right)=0$ be boundary conditions of rank $m$. Then boundary conditions $\mathbf{u}^{*}\left(\boldsymbol{x}_{a}, \mathbf{x}_{b}\right)=0$, where $\boldsymbol{u}^{*}\left(\mathbf{y}_{a}, \mathbf{y}_{b}\right)$ are boundary forms of rank $2 n-m$ determined as in Theorem 1.2 are called adjoint boundary value conditions.

Theorem 1.3. Boundary conditions

$$
\mathbf{u}^{*}\left(\mathbf{x}_{a}, \mathbf{x}_{b}\right) \equiv \boldsymbol{P} \mathbf{x}(a)+\mathbf{Q} \mathbf{x}(b)=0
$$

of rank $2 n-m$ are adjoint to boundary conditions (1.2) of rank $m$ if and only if

$$
-M P^{\prime}+N Q^{\prime}=0 \text {. }
$$

If $\mathbf{u}\left(\mathbf{x}_{\boldsymbol{a}}, \mathbf{x}_{\boldsymbol{b}}\right)$ is a system of $m$ boundary forms of rank $m$, then the problem of finding on $\langle a, b\rangle$ solutions of the system

$$
I(x) \equiv \dot{x}+A(t) x=0, \quad u\left(x_{a}, x_{b}\right)=0
$$

is called a homogeneous boundary value problem $(\lambda)$ of rank $m$.

The problem of finding solutions of the system

$$
I *(\mathbf{x})=0, \quad \mathbf{u}^{*}\left(\mathbf{x}_{a}, \mathbf{x}_{b}\right)=0
$$

on $\langle a, b\rangle$ is called the boundary value problem $\left(\lambda^{*}\right)$ adjoint to $(\lambda)$.

Theorem 1.4. Let $\Phi(t)$ be a fundamental matrix of solutions of (1.1). Then the problem $(\lambda)$ has exactly $k(0 \leqq k \leqq n)$ linearly independent solutions if and only if the matrix $\mathbf{M} \boldsymbol{\Phi}(a)+\mathbf{N} \boldsymbol{\Phi}(b)$ is of rank $n-k$.

Theorem 1.5. If the problem $(\lambda)$ has exactly $k$ linearly independent solutions, then the problem $\left(\lambda^{*}\right)$ has exactly $k+m-n$ linearly independent solutions.

The problem of finding on $\langle a, b\rangle$ solutions of

$$
I(x)=f, \quad u\left(x_{a}, x_{b}\right)=\gamma
$$


where $f(t)$ is of class $C^{0}$ on $\langle a, b\rangle$ and $\gamma$ is a constant $m$-vector (while $f \neq \equiv$ and $\gamma \neq 0$ ) is called a nonhomogeneous boundary value problem associated with $(\lambda)$. (If $\gamma=0$ then the problem is called semi-homogeneous.)

Theorem 1.6. The nonhomogeneous problem (1.7) has a solution if and only if

$$
\int_{a}^{b} f(t) \cdot \psi(t) \mathrm{d} t=\gamma \cdot \tilde{u}^{*}\left(\psi_{a}, \psi_{b}\right)
$$

for every solution $\psi(t)$ of the adjoint boundary value problem $\left(\lambda^{*}\right)$. (For a semihomogeneous problem the right-hand side equals zero, of course.)

Remark 1.2. According to relation (1.6) and to equality $u^{*}\left(\psi_{a}, \psi_{b}\right)=0$ for every solution $\psi(t)$ of $\left(\lambda^{*}\right)$ the condition (1.8) is evidently invariant with respect to the choice of the complementary boundary value conditions to $\mathbf{u}\left(\mathbf{x}_{a}, \mathbf{x}_{b}\right)=0$.

\section{AUXILIARY THEOREMS}

Theorem 2.1. (Variation-of-constants method for nonlinear systems.) Consider the system

$$
\dot{x}=f(t, x)+\varepsilon g(t, x, \varepsilon),
$$

where $\mathbf{x}, \boldsymbol{f}$ and $\mathbf{g}$ are $n$-vectors.

Let $\mathfrak{I}$ be a one-dimensional closed bounded interval $\langle a, b\rangle$. Let $\boldsymbol{\eta}_{0}(t)=\boldsymbol{\eta}\left(t, \boldsymbol{c}_{0}\right)$ be a solution of

$$
\dot{y}=f(t, y)
$$

with an initial value $\boldsymbol{\eta}\left(a, \boldsymbol{c}_{0}\right)=\boldsymbol{c}_{0}$ defined on $\mathfrak{I}$. Let

$$
\mathfrak{X}=E\left[\mathbf{x} \mid\left\|\mathbf{x}-\boldsymbol{\eta}_{0}(t)\right\|<\varrho, t \in \mathfrak{I}\right], \varrho>0 .
$$

Let $\leftleftarrows$ be a one-dimensional interval $\left\langle 0, \varepsilon_{0}\right\rangle, \varepsilon_{0}>0$.

Let $\boldsymbol{f}(t, \mathbf{x}) \in C^{0.1 L}$ on $\mathfrak{I} \times \mathfrak{X}$ and $\mathbf{g}(t, \mathbf{x}, \varepsilon) \in C^{0,0 L, 0}$ on $\mathfrak{T} \times \mathfrak{X} \times \mathfrak{E}$. Let $\mathfrak{E}=$ $=E\left[\mathbf{c} \mid\left\|\boldsymbol{c}-\boldsymbol{c}_{0}\right\|<\mu_{0}\right], \mu_{0}>0$, be such that every solution $\boldsymbol{\eta}(t, \boldsymbol{c})$ of $(2.2)$ with $\boldsymbol{\eta}(a, c)=\boldsymbol{c} \in \mathfrak{C}$ remains in $\mathfrak{X}$ for $t \in \mathfrak{T}$.

Then there exist $\mathfrak{E}_{1}=\left\langle 0, \varepsilon_{1}\right\rangle, 0<\varepsilon_{1} \leqq \varepsilon_{0}$ and $\mathfrak{E}_{1}=E\left[\mathbf{c} \mid\left\|\mathbf{c}-c_{0}\right\|<\mu_{1}\right]$, $0<\mu_{1} \leqq \mu_{0}$ such that every solution $\xi(t, \mathbf{c}, \varepsilon)$ of $(2.1)$ where $\varepsilon \in \mathfrak{F}_{1}$ with $\xi(a, \mathbf{c}, \varepsilon)=$ $=c \in \mathfrak{S}_{1}$ remains for $t \in \mathfrak{I}$ in $\mathfrak{X}$ and is given by the formula

$$
\boldsymbol{\xi}(t, \boldsymbol{c}, \varepsilon)=\boldsymbol{\eta}(t, \boldsymbol{C}(t, \boldsymbol{c}, \varepsilon)),
$$

where $\mathbf{C}(t, \boldsymbol{c}, \varepsilon)$ is the solution of the integral equation

$$
\boldsymbol{\Gamma}(t)=\boldsymbol{c}+\varepsilon \int_{a}^{t} \boldsymbol{\eta}_{\mathrm{c}}^{-1}(\tau, \boldsymbol{\Gamma}(\tau)) \mathbf{g}(\tau, \boldsymbol{\eta}(\tau, \boldsymbol{\Gamma}(\tau)), \varepsilon) \mathrm{d} \tau .
$$

The solution $\xi(t, c, \varepsilon)$ is of class $C^{1,0 L, 0}$ on $\mathfrak{T} \times \mathfrak{C}_{1} \times \mathfrak{F}_{1}$. 
Proof. By the existence theorem and by the theorem on continuous dependence of solutions on initial conditions solutions $\boldsymbol{\eta}(t, \boldsymbol{c})$ of $(2.2)$ for $t \in \mathfrak{T}, \boldsymbol{c} \in \mathfrak{E}$ are of class $C^{1,1 L}$. Since

$$
\frac{\mathrm{d}}{\mathrm{d} t}\left(\frac{\mathbf{D} \boldsymbol{\eta}}{\mathbf{D} \mathbf{c}}(t, \mathbf{c})\right)=\frac{\mathbf{D f}}{\mathbf{D x}}(\mathrm{t}, \boldsymbol{\eta}(\mathrm{t}, \mathbf{c})) \frac{\mathbf{D} \boldsymbol{\eta}}{\mathbf{D c}}(\mathrm{t}, \mathbf{c}),
$$

by the known formula

$$
\operatorname{det}\left(\frac{\mathbf{D} \boldsymbol{\eta}}{\mathbf{D} \mathbf{c}}(\mathrm{t}, \mathbf{c})\right)=\operatorname{det}\left(\frac{\mathbf{D} \boldsymbol{\eta}}{\mathbf{D} \boldsymbol{c}}(a, \boldsymbol{c})\right) \exp \int_{a}^{t}\left(\sum_{j=1}^{n} \frac{\partial f_{j}}{\partial x_{j}}(\tau, \boldsymbol{\eta}(\tau, \boldsymbol{c}) \mathrm{d} \tau) .\right.
$$

Since $\operatorname{det}\left(\boldsymbol{\eta}_{\boldsymbol{c}}(a, \boldsymbol{c})\right)=1$ and the integrand is for every $(\tau, \boldsymbol{c}) \in \mathfrak{Z} \times \mathfrak{S}$ finite, $\operatorname{det}(\boldsymbol{\eta}(t, \boldsymbol{c})) \neq 0$ and the inverse matrix $\boldsymbol{\eta}_{\boldsymbol{c}}^{-1}(t, \boldsymbol{c})$ exists for $(t, \boldsymbol{c}) \in \mathfrak{Z} \times \mathfrak{C}$.

Hence, the right-hand side of (2.4) is for $(\tau, \Gamma) \in \mathfrak{I} \times \mathfrak{C}$ defined and satisfies a Lipschitz condition in $\boldsymbol{\Gamma}$. Therefore by the existence and uniqueness theorem and by the theorem on the continuous dependence of solutions on initial conditions and parameters there exist sets $\mathfrak{F}_{1}$ and $\mathfrak{S}_{1}$ mentioned above such that solutions $\boldsymbol{C}(t, \boldsymbol{c}, \varepsilon)$ of (2.4), where $\varepsilon \in \mathfrak{E}$, exist, are uniquely determined and $\boldsymbol{C}(t, \boldsymbol{c}, \varepsilon) \in \mathfrak{V}$ for $t \in \mathfrak{T}, \boldsymbol{c} \in \mathfrak{S}_{1}$ and $\varepsilon \in \mathfrak{E}_{1}$. Hence $\boldsymbol{\eta}(t, \boldsymbol{C}(t, \boldsymbol{c}, \varepsilon))$ is defined on $\mathfrak{I} \times \mathfrak{S}_{1} \times \mathfrak{E}_{1}$, too. According to $\boldsymbol{\eta}(a, \boldsymbol{c})=\boldsymbol{c}$ and $(2.4) \boldsymbol{\eta}(a, \boldsymbol{C}(a, \boldsymbol{c}, \varepsilon))=\boldsymbol{c}$.

By differentiating the function $\boldsymbol{\eta}(t, \boldsymbol{C}(t, \boldsymbol{c}, \varepsilon))$ we find easily that it satisfies (2.1). Since the solution $\xi(t, c, \varepsilon)$ of $(2.1)$ is by the assumptions of the theorem uniquely determined by initial conditions, the relation (2.3) as well as the whole assertion of the theorem readily follows.

Let us recall the following two theorems:

Theorem 2.2. (Mean value theorem.) Let $\boldsymbol{f}(t, \mathbf{x}, \varepsilon)$ be a k-vector function defined and of class $C^{0,1,1}$ on the set $\mathfrak{I} \times \mathfrak{X} \times \mathfrak{E}$ where $\mathfrak{I}=\langle a, b\rangle, \mathfrak{X}$ is a convex domain in $E^{n}$, $\left.E=\left\langle 0, \varepsilon_{0}\right\rangle, \varepsilon_{0}\right\rangle 0$.

Then for arbitrary $t \in \mathfrak{I}, \mathbf{x}, \mathbf{x}+\boldsymbol{h} \in \mathfrak{X}$ and $\varepsilon \in \mathfrak{E}$ there holds

$$
\begin{aligned}
f(t, \mathbf{x}+\boldsymbol{h}, \varepsilon)= & \boldsymbol{f}(t, \mathbf{x}, 0)+\int_{0}^{1} \frac{\mathbf{D} f}{\mathbf{D x}}(t, \mathbf{x}+\vartheta \boldsymbol{h}, \vartheta \varepsilon) \mathrm{d} \vartheta \boldsymbol{h}+ \\
& +\int_{0}^{1} \frac{\partial f}{\partial \varepsilon}(t, \mathbf{x}+\vartheta \boldsymbol{h}, \vartheta \varepsilon) \mathrm{d} \vartheta \varepsilon .
\end{aligned}
$$

Theorem 2.3. (Implicit function theorem.) Let $\mathfrak{w}(c, \varepsilon)$ be a $k$-vector function defined and of class $C^{p, p}(p \geqq 1)$ on $\mathfrak{N}\left(c_{0}, \delta\right) \times$ where $\mathfrak{N}\left(c_{0}, \delta\right)$ is a neighborhood of the point $\boldsymbol{c}_{0} \in E^{k}$ and $\left.\xi=\left\langle 0, \varepsilon_{0}\right\rangle, \varepsilon_{0}\right\rangle 0$. Let

$$
\mathfrak{w}\left(\boldsymbol{c}_{0}, 0\right) \equiv 0
$$

and

$$
\operatorname{det}\left(\frac{\mathbf{D}_{\mathfrak{w}}}{\mathbf{D} \boldsymbol{c}}\left(\boldsymbol{c}_{0}, 0\right)\right) \neq 0
$$


Then there exists an interval $\mathfrak{\Xi}_{1}=\left\langle 0, \varepsilon_{1}\right\rangle, 0<\varepsilon_{1} \leqq \varepsilon_{0}$ such that for $\varepsilon \in \mathfrak{F}_{1}$ there exists a unique solution $c=c^{*}(\varepsilon) \in C^{p}$ of the system

$$
\mathfrak{w}(c, \varepsilon)=0
$$

such that $c^{*}(0)=c_{0}$.

Theorem 2.4. Consider the equation

$$
\mathbf{w}(\boldsymbol{c}, \varepsilon) \equiv \boldsymbol{u}(\boldsymbol{c})+\varepsilon \mathbf{v}(\boldsymbol{c}, \varepsilon)=0,
$$

where $\mathbf{u}, \mathbf{v}$ and $\mathbf{w}$ are $n$-vectors and $\varepsilon$ is a scalar.

Let the equation $\mathbf{u}\left(\boldsymbol{c}_{0}\right)=0$ have a real solution $\boldsymbol{c}_{0}=\mathbf{c}_{0}^{*}$. Let $\mathfrak{S}_{0}=E[\mathbf{c} \mid \| \mathbf{c}-$ $\left.-c_{0} \|<\mu\right], \mu>0, \mathbb{F}=\left\langle 0, \varepsilon_{0}\right\rangle, \varepsilon_{0}>0$. Let $\mathbf{u}(\mathbf{c}) \in C^{1 L}$ and $\mathbf{v}(\boldsymbol{c}, \varepsilon) \in C^{0 L, 0}$ for $\boldsymbol{c} \in \mathfrak{W}$, $\varepsilon \in$ E. Let

$$
\operatorname{det}\left(\frac{\mathbf{D u}}{\mathbf{D} \boldsymbol{c}}\left(\mathbf{c}_{0}^{*}\right)\right) \neq 0 .
$$

Then there exists $\varepsilon_{1}, 0<\varepsilon_{1} \leqq \varepsilon_{0}$ such that there exists for each $\varepsilon \in \mathfrak{F}_{1}=\left\langle 0, \varepsilon_{1}\right\rangle$ the unique solution $c=c^{*}(\varepsilon)$ of $(2.9)$, which tends to $c_{0}^{*}$ as $\varepsilon \rightarrow 0$.

The proof can easily be performed by help of the successive approximations method.

\section{GENERAL NONLINEAR TWO-POINT BOUNDARY VALUE PROBLEMS}

We shall begin by considering the simple case when the shortened boundary value problem has a unique (isolated) solution.

Theorem 3.1. Let the interval $\mathfrak{I}=\langle a, b\rangle$ be given. Consider the boundary value problem (v)

$$
\begin{aligned}
\dot{\boldsymbol{x}} & =\boldsymbol{f}(\mathrm{t}, \boldsymbol{x})+\varepsilon \mathbf{g}(\mathrm{t}, \boldsymbol{x}, \varepsilon) \\
\mathbf{w}\left(\boldsymbol{x}_{a}, \boldsymbol{x}_{b}, \varepsilon\right) & \equiv \mathbf{u}\left(\boldsymbol{x}_{a}, \boldsymbol{x}_{b}\right)+\varepsilon \mathbf{v}\left(\boldsymbol{x}_{a}, \boldsymbol{x}_{b}, \varepsilon\right)=0,
\end{aligned}
$$

where $\mathbf{x}, \boldsymbol{f}, \mathbf{g}, \boldsymbol{u}, \mathbf{v}$ and $\mathbf{w}$ are n-vectors and $\varepsilon$ is a scalar "small" parameter (as before $\left.\mathbf{x}_{a}=\mathbf{x}(a), \boldsymbol{x}_{b}=\mathbf{x}(b)\right)$. Let the following assumptions be fulfilled.

(I) Let $\mathfrak{P}$ and $\mathfrak{Q}$ be open sets in $E^{n}$ and let $\mathfrak{F}=\left\langle 0, \varepsilon_{0}\right\rangle, \varepsilon_{0}>0$. Let $\mathbf{u}(\boldsymbol{p}, \boldsymbol{q}) \in C^{1 L, 1 L}$ and $\mathbf{v}(\boldsymbol{p}, \boldsymbol{q}, \varepsilon) \in C^{0 L, 0 L, 0}$ for $\boldsymbol{p} \in \mathfrak{P}, \boldsymbol{q} \in \mathfrak{Q}$, and $\varepsilon \in \mathfrak{E}$.

(II) Let the system

$$
\dot{\mathbf{y}}=\boldsymbol{f}(t, \mathbf{y})
$$

have solutions $\boldsymbol{\eta}(t, \boldsymbol{c})$ with $\boldsymbol{\eta}(a, c)=\boldsymbol{c}$ existing and staying in $\mathfrak{X}$ for $t \in \mathfrak{Z}$ and for all $\mathbf{c} \in \mathfrak{C}$, $\mathfrak{C}$ an open set in $E^{n}$.

Let the boundary value problem $\left(v_{0}\right)$ given by (3.3) and

$$
\boldsymbol{u}\left(\mathbf{y}_{a}, \mathbf{y}_{b}\right)=0
$$


have a solution $\eta\left(t, c_{0}^{*}\right)$ where $c_{0}^{*} \in \mathfrak{S}$ (so that $c_{0}^{*}$ is a solution of

$$
\boldsymbol{u}(\boldsymbol{\eta}(a, c), \boldsymbol{\eta}(b, \boldsymbol{c}))=0 .)
$$

Let $\boldsymbol{\eta}^{*}(t)$ denote $\boldsymbol{\eta}\left(t, \mathbf{c}_{\mathbf{0}}^{*}\right)$. Let $\boldsymbol{\eta}_{a}^{*} \in \mathfrak{P}, \boldsymbol{\eta}_{b}^{*} \in \mathfrak{Q}$.

(III) Let the variational boundary value problem $(\lambda)$

$$
\dot{z}=f_{x}\left(t, \eta^{*}(t)\right) z,
$$

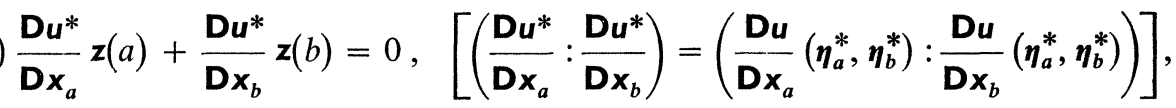

have the trivial solution only.

(IV) Denote $\mathfrak{X}=E\left[\mathbf{x} \mid\left\|\mathbf{x}-\boldsymbol{\eta}^{*}(t)\right\|<\varrho, t \in \mathfrak{T}\right], \varrho>0$. Let $\boldsymbol{f}(t, \mathbf{x}) \in C^{0,1 L}$ on $\mathfrak{I} \times \mathfrak{X}$ and $\mathbf{g}(t, \boldsymbol{x}, \varepsilon) \in C^{0,0 L, 0}$ on $\mathfrak{I} \times \mathfrak{X} \times \mathfrak{F}$.

Then there exists for sufficiently small $\varepsilon>0$ a unique solution $\xi\left(t, c^{*}(\varepsilon), \varepsilon\right)$ (with $\left.\xi\left(a, c^{*}(\varepsilon), \varepsilon\right)=c^{*}(\varepsilon)\right)$ of the problem $(v)$ which tends to the solution $\eta^{*}(t)$ of $\left(v_{0}\right)$ as $\varepsilon \rightarrow 0$.

Proof. By Theorem 2.1 there exist an open set $\mathfrak{S}_{1} \subset \mathfrak{C}$ and a set $\mathfrak{F}_{1}=\left\langle 0, \varepsilon_{1}\right\rangle$, $0<\varepsilon_{1} \leqq \varepsilon_{0}$, such that every solution $\xi(t, c, \varepsilon)$ of $(3.1)$ where $\varepsilon \in \mathbb{E}_{1}$ with $\xi(a, c, \varepsilon)=$ $=c \in \mathfrak{S}_{1}$ stays in $\mathfrak{X}$ for $t \in \mathfrak{Z}$ and that $\xi(a, c, \varepsilon) \in \mathfrak{P}, \xi(b, c, \varepsilon) \in \mathfrak{Q}$ for $c \in \mathfrak{S}_{1}, \varepsilon \in \mathfrak{E}_{1}$. By the same theorem the solution $\xi(t, c, \varepsilon)$ may be written for $c \in \mathfrak{S}_{1}$ and $\varepsilon \in \mathfrak{F}_{1}$ in the form (2.3) where $\boldsymbol{C}(t, \boldsymbol{c}, \varepsilon)$ is the solution of $(2.4)(\boldsymbol{\eta}(t, \boldsymbol{c})$ has of course the same meaning as in the assumption (II)).

Inserting (2.3) into (3.2) we get

$$
\boldsymbol{u}(\boldsymbol{\eta}(a, \boldsymbol{c}), \quad \boldsymbol{\eta}(b, \boldsymbol{C}(b, \boldsymbol{c}, \varepsilon))+\varepsilon \boldsymbol{v}(\boldsymbol{\eta}(a, \boldsymbol{c}), \quad \boldsymbol{\eta}(b, \boldsymbol{C}(b, \boldsymbol{c}, \varepsilon)), \varepsilon)=0 .
$$

This is a system of $n$ necessary and sufficient conditions for components of the initial vector $\boldsymbol{c}=\boldsymbol{c}(\varepsilon)$ such that $\xi(t, \boldsymbol{c}(\varepsilon), \varepsilon)$ be a solution of $(v)$.

Letting in (3.8) $\varepsilon \rightarrow 0$ we get (denoting $c(0)=c_{0}$ )

$$
\boldsymbol{u}\left(\boldsymbol{\eta}\left(a, \boldsymbol{c}_{0}\right), \quad \boldsymbol{\eta}\left(b, \boldsymbol{c}_{0}\right)\right)=0 .
$$

By the assumption (II) this equation has a real solution $c_{0}=c_{0}^{*}$. Let us show that the jacobian of (3.9) is nonvanishing at the point $c_{0}=c_{0}^{*}$.

Indeed, $\boldsymbol{\eta}_{\boldsymbol{c}}\left(t, \boldsymbol{c}_{0}^{*}\right)$ being a fundamental matrix of solutions of (3.6), the matrix

$$
\boldsymbol{U}\left(c_{0}^{*}\right) \equiv \frac{\mathrm{Du}}{\mathrm{D} \boldsymbol{x}_{a}}\left(\boldsymbol{\eta}_{a}^{*}, \boldsymbol{\eta}_{b}^{*}\right) \frac{\mathrm{D} \boldsymbol{\eta}_{a}^{*}}{\mathrm{Dc}}+\frac{\mathrm{Du}}{\mathrm{D} \boldsymbol{x}_{b}}\left(\boldsymbol{\eta}_{a}^{*}, \boldsymbol{\eta}_{b}^{*}\right) \frac{\mathrm{D} \boldsymbol{\eta}_{b}^{*}}{\mathrm{Dc}}
$$

has to be (by the assumption (III) and by Theorem 1.4) of rank $n$, which means that $\operatorname{det} \boldsymbol{U}\left(c_{0}^{*}\right) \neq 0$. But $\operatorname{det}\left(\boldsymbol{U}\left(\boldsymbol{c}_{0}\right)\right)$ is evidently the jacobian of (3.9) with respect to $\boldsymbol{c}_{0}$ at the point $c=c_{0}^{*}$.

Denote

$$
\mathfrak{g}=\int_{a}^{b} \boldsymbol{\eta}_{\mathbf{c}}^{-1}(\tau, \boldsymbol{C}(\tau, \boldsymbol{c}, \varepsilon)) \mathbf{g}(\tau, \boldsymbol{\eta}(\tau, \boldsymbol{C}(\tau, \boldsymbol{c}, \varepsilon), \varepsilon) \mathrm{d} \tau
$$


and applying the mean value theorem write (3.8) in the form

$$
\begin{aligned}
& \boldsymbol{u}\left(\boldsymbol{\eta}(a, \boldsymbol{c}), \boldsymbol{\eta}(b, \boldsymbol{c})+\varepsilon\left[\int _ { 0 } ^ { 1 } \left(\frac{\mathbf{D u}}{\mathbf{D x}_{b}}(\boldsymbol{\eta}(a, \boldsymbol{c}), \boldsymbol{\eta}(b, \boldsymbol{c}+\vartheta \varepsilon \mathfrak{g}) .\right.\right.\right. \\
& \left.\frac{\mathbf{D} \boldsymbol{\eta}}{\mathbf{D} \boldsymbol{c}}(b, \boldsymbol{c}+\vartheta \varepsilon \mathfrak{g}) \mathrm{d} \vartheta \mathfrak{g}+\boldsymbol{v}(\boldsymbol{\eta}(a, \boldsymbol{c}), \boldsymbol{\eta}(b, \boldsymbol{c}+\varepsilon \mathfrak{g}, \varepsilon))\right]=0 .
\end{aligned}
$$

By the assumptions (I), (II) and (IV) and by the assertion of Theorem 2.1 the equation $\left(3.8^{\prime}\right)$ fulfils all assumptions of Theorem 2.4. Hence the assertion of Theorem 3.1 immediately follows.

From Theorem 3.1 we get the following corollaries for quasi-linear boundary value problems and for periodic boundary conditions.

(We use the same notations as before.)

Corollary 3.1. Let the interval $\mathfrak{I}=\langle a, b\rangle$ be given. Consider the boundary value problem

$$
\begin{gathered}
\dot{\mathbf{x}}=\mathbf{A}(t) \mathbf{x}+\varepsilon \mathbf{g}(t, \mathbf{x}, \varepsilon), \\
\mathbf{M} \mathbf{x}(a)+\mathbf{N} \mathbf{x}(b)+\varepsilon \mathbf{v}\left(\mathbf{x}_{a}, \mathbf{x}_{b}, \varepsilon\right)=0
\end{gathered}
$$

where $\mathbf{A}(t), \mathbf{M}$ and $\mathbf{N}$ are $n \times n$ matrices, the two latter being constant.

Let the following assumptions be fulfilled.

(I) Let $\chi(\boldsymbol{M}: \mathbf{N})=n$. Let $\mathbf{v}(\mathbf{p}, \boldsymbol{q}, \varepsilon) \in C^{0 L, 0 L, 0}$ for $\mathbf{p} \in \mathfrak{P}, \boldsymbol{q} \in \mathfrak{Q}$ and $\varepsilon \in \mathfrak{F}$. Let $0 \in \mathfrak{P}, 0 \in \mathfrak{D}^{\text {. }}$.

(II) Let the boundary value problem

$$
\begin{gathered}
\dot{\mathbf{y}}=\mathbf{A}(t) \mathbf{y}, \\
\mathbf{M} \mathbf{y}(a)+\mathbf{N} \mathbf{y}(b)=0
\end{gathered}
$$

have only the trivial solution.

(III) Denote $\mathfrak{X}_{0}=E[\mathbf{x} \mid\|\mathbf{x}\|<\varrho], \varrho>0$. Let $\mathbf{A}(t) \in C^{0}$ for $t \in \mathfrak{Z}$ and $\mathbf{g}(t, \mathbf{x}, \varepsilon) \in$ $\in C^{0,0 L, 0}$ on $\mathfrak{T} \times \mathfrak{X}_{0} \times \mathfrak{F}$.

Then there exists for sufficiently small $\varepsilon>0$ a unique solution $\xi\left(t, c^{*}(\varepsilon), \varepsilon\right)$ of the boundary value problem (3.10), (3.11), which tends to the trivial solution of the boundary value problem (3.12) as $\varepsilon \rightarrow 0$.

Corollary 3.2. Consider the system (3.1). Let the following assumptions be fulfilled.

(I) Let $\mathbf{f}$ and $\mathbf{g}$ be periodic in $t$ with period $\omega$ (in the sequel briefly: $\omega$-periodic).

(II) Let the system (3.3) have a $\omega$-periodic solution $\boldsymbol{\eta}\left(t, c_{0}^{*}\right)=\boldsymbol{\eta}^{*}(t)$.

(III) Let the system (3.6) have no w-periodic solution (except the trivial one).

(IV) Denote $\mathfrak{I}_{\omega}=\langle 0, \omega\rangle$. Let $\boldsymbol{f}(t, \mathbf{x}) \in C^{0,1 L}$ on $\mathfrak{I}_{\omega} \times \mathfrak{X}$ and $\mathbf{g}(t, \mathbf{x}, \varepsilon) \in C^{0,0 L, 0}$ on $\mathfrak{Z}_{\omega} \times \mathfrak{X} \times \mathfrak{F}$. 
Then there exists for sufficiently small $\varepsilon>0$ a unique $\omega$-periodic solution $\xi(t$, $\left.c^{*}(\varepsilon), \varepsilon\right)$ of (3.1) which tends to $\boldsymbol{\eta}^{*}(t)$ as $\varepsilon \rightarrow 0$.

In the book [2] an analogous theorem is proved for the system $\dot{\boldsymbol{x}}=\boldsymbol{f}(t, \mathbf{x}, \varepsilon)$ where $f(t, \mathbf{x}, \varepsilon) \in C^{0,1,0}$ on $\mathfrak{I}_{\omega} \times \mathfrak{X} \times \mathfrak{E}$.

Let us now investigate the more interesting and more difficult case when the shortened boundary value problem has a family of solutions depending on $k$ parameters, $1 \leqq k \leqq n$.

Theorem 3.2. Let an interval $\mathfrak{Z}=\langle a, b\rangle$ be given. Consider the boundary value problem (v) given by (3.1), (3.2). Let the following assumptions be fulfilled.

(I) (a) Let $\mathbf{u}(\boldsymbol{p}, \boldsymbol{q}) \in C^{2,2}$ and $\mathbf{v}(\boldsymbol{p}, \boldsymbol{q}, \varepsilon) \in C^{1,1,1}$ for $\boldsymbol{p} \in \mathfrak{P}, \boldsymbol{q} \in \mathfrak{Q}$, and $\varepsilon \in \mathfrak{E}$, where $\mathfrak{P}$ and $\mathfrak{Q}$ are open sets in $E^{n}$ and $\left.\leftleftarrows=\left\langle 0, \varepsilon_{0}\right\rangle, \varepsilon_{0}\right\rangle 0$.

(b) Let for $\boldsymbol{p} \in \mathfrak{P}, \boldsymbol{q} \in \mathfrak{D}$.

$$
\chi\left(\frac{\mathbf{D u}}{\mathbf{D} \mathbf{x}_{a}}(\boldsymbol{p}, \boldsymbol{q}): \frac{\mathbf{D u}}{\mathbf{D} \mathbf{x}_{b}}(\boldsymbol{p}, \boldsymbol{q})\right)=n .
$$

(II) (a) Denote $\sqrt{5}$ an open set in $E^{n}$. Let there exist solutions $\boldsymbol{\eta}(t, c)$ of (3.3) defined and remaining in $\mathfrak{X}$ for $t \in \mathfrak{Z}$ and for every $\boldsymbol{c} \in \mathfrak{C}$.

(b) Let the equations

$$
\mathbf{u}(\boldsymbol{\eta}(a, c), \boldsymbol{\eta}(b, \boldsymbol{c}))=0
$$

have a real solution $\mathbf{c}_{\bar{\gamma}}=\sigma_{0}\left(\boldsymbol{c}_{\mathscr{\gamma}}\right), \mathscr{V} \subset \mathscr{N}, v(\mathscr{V})=k, 1 \leqq k \leqq n$.

(c) Denote $c_{\gamma}=\gamma$ and $\varrho_{0}(\gamma)=\gamma+\sigma_{0}(\gamma)$. Let $\sigma_{0}(\gamma) \in C^{2}$ for $\gamma \in(\mathfrak{S}$, ( \&) an open set in $E^{k}$, and let $\mathfrak{D}=E\left[\mathbf{c} \mid \mathbf{c}=\varrho_{0}(\gamma), \gamma \in \mathfrak{S}\right] \subset(\mathfrak{S}$.

Denote $\tilde{\boldsymbol{\eta}}(t)=\boldsymbol{\eta}\left(t, \varrho_{0}(\gamma)\right)$. Thus $\tilde{\boldsymbol{\eta}}(t)$ is a k-parametric solution of the boundary value problem $\left(v_{0}\right)$ given by (3.3), (3.4).

(d) Let $\tilde{\boldsymbol{\eta}}(a) \in \mathfrak{P}, \tilde{\boldsymbol{\eta}}(b) \in \mathfrak{D}$, for $\boldsymbol{\gamma} \in \mathfrak{G}$.

(III) Let the variational boundary value problem $(\lambda)$

$$
\begin{gathered}
\dot{\mathbf{z}}=\boldsymbol{f}_{\mathbf{x}}(t, \tilde{\boldsymbol{\eta}}(t)) \mathbf{z}, \\
\frac{\mathbf{D} \tilde{u}}{\mathbf{D} \mathbf{x}_{\boldsymbol{a}}} \mathbf{z}(a)+\frac{\mathbf{D} \tilde{u}}{\mathbf{D x}_{\boldsymbol{b}}} \mathbf{z}(b)=0,
\end{gathered}
$$

where

$$
\left(\frac{\mathrm{Du}}{\mathrm{D} \mathbf{x}_{a}}: \frac{\mathrm{D} \tilde{u}}{\mathrm{Dx}}\right)=\left(\frac{\mathrm{Du}}{\mathrm{Dx}_{\boldsymbol{a}}}\left(\tilde{\boldsymbol{\eta}}_{a}, \tilde{\boldsymbol{\eta}}_{b}\right): \frac{\mathrm{Du}}{\mathrm{Dx}_{\boldsymbol{b}}}\left(\tilde{\boldsymbol{\eta}}_{a}, \tilde{\boldsymbol{\eta}}_{b}\right)\right),
$$

have exactly $k$ linearly independent solutions for $\gamma \in(\mathfrak{S}$.

We shall show that there exist a set $\mathscr{S} \subset \mathscr{N}, v(\mathscr{S})=k$, a set $\mathfrak{G}_{0}, \mathfrak{G H}_{0}$ a domain in $\mathcal{B}$ and a $k \times(n-k)$ matrix $\mathbf{T}(\gamma)$ such that

$$
\frac{\mathrm{D} \tilde{\mathbf{u}}_{\mathscr{S}}}{\mathrm{Dc}}-\mathbf{T}(\gamma) \frac{\mathrm{D} \tilde{\mathbf{u}}_{\overline{\mathcal{S}}}}{\mathrm{Dc}} \equiv 0
$$

for $\gamma \in \mathfrak{G S}_{0}$. 
Further, we shall show that there exist a set $\mathfrak{G}_{1}, \mathfrak{G}_{1}$ a domain in $\mathfrak{G}_{0}, a k \times n$ matrix $\Psi(t, \gamma)$ whose rows are formed by solutions of a boundary value problem $\left(\lambda^{*}\right)($ adjoint to $(\lambda))$

$$
\begin{gathered}
\dot{\boldsymbol{w}}^{\prime}=-\mathbf{w}^{\prime} \boldsymbol{f}_{\mathbf{x}}(t, \tilde{\boldsymbol{\eta}}(t)), \\
\mathbf{w}^{\prime}(a) \mathbf{P}^{\prime}(\gamma)+\mathbf{w}^{\prime}(b) \mathbf{Q}^{\prime}(\gamma)=0
\end{gathered}
$$

where $n \times n$ matrices $\mathbf{P}(\gamma)$ and $\mathbf{Q}(\gamma)$ satisfy conditions

$$
-\frac{\mathbf{D} \tilde{u}}{\mathbf{D x}_{a}} \mathbf{P}^{\prime}(\gamma)+\frac{\mathbf{D} \tilde{u}}{\mathbf{D x}_{b}} \mathbf{Q}^{\prime}(\gamma) \equiv 0, \quad \chi(\mathbf{P}(\gamma): \mathbf{Q}(\gamma))=n,
$$

for $\gamma \in \mathfrak{G H}_{1}$ and which fulfils the condition

(IV) (a) Let the system

$$
\boldsymbol{\Psi}(b, \gamma)=\frac{\mathbf{D} \tilde{\boldsymbol{u}}_{\mathscr{y}}}{\mathbf{D} \boldsymbol{x}_{b}}-\boldsymbol{T}(\gamma) \frac{\mathbf{D} \tilde{\mathbf{u}}_{\overline{\mathcal{S}}}}{\mathbf{D x _ { b }}} .
$$

$$
\int_{a}^{b} \boldsymbol{\Psi}\left(s, \gamma_{0}\right) \boldsymbol{g}\left(s, \boldsymbol{\eta}\left(s, \varrho_{0}\left(\gamma_{0}\right)\right), 0\right) \mathrm{d} s+\tilde{\mathbf{v}}_{0 \mathscr{S}}-\boldsymbol{T}\left(\gamma_{0}\right) \tilde{\mathbf{v}}_{0 \overline{\mathscr{S}}}=0
$$

where $\tilde{\mathbf{v}}_{0}=\mathbf{v}\left(\boldsymbol{\eta}\left(a, \varrho_{0}\left(\gamma_{0}\right)\right), \boldsymbol{\eta}\left(b, \varrho_{0}\left(\gamma_{0}\right), 0\right)\right.$ have a real solution $\gamma_{0}=\gamma_{0}^{*}$.

(b) Let $\gamma_{0}^{*} \in \mathfrak{G}_{1}$.

(c) Let the jacobian of (3.21) with respect to $\gamma_{0}$ be nonvanishing at the point $\gamma_{0}=\gamma_{0}^{*}$.

(V) Denote $\mathfrak{X}=E\left[\mathbf{x} \mid\left\|\mathbf{x}-\boldsymbol{\eta}\left(t, \mathrm{c}_{0}^{*}\right)\right\|<\varrho, t \in \mathfrak{X}\right], \varrho>0$, where $c_{0}^{*}=\varrho_{0}\left(\gamma_{0}^{*}\right)$. Let $\boldsymbol{f}(t, \mathbf{x}) \in C^{0,2}$ and $\mathbf{g}(t, \mathbf{x}, \varepsilon) \in C^{0,1,1}$ for $t \in \mathfrak{I}, \mathbf{x} \in \mathfrak{X}, \varepsilon \in \mathfrak{E}$.

Then there exists for sufficiently small $\varepsilon>0$ a unique solution $\xi\left(t, c^{*}(\varepsilon), \varepsilon\right)$ of the problem $(v)$ with $\xi\left(a, c^{*}(\varepsilon), \varepsilon\right)=c^{*}(\varepsilon) \in C^{1}$, which tends to the solution $\boldsymbol{\eta}\left(t, c_{0}^{*}\right)$ of the problem $\left(v_{0}\right)$ as $\varepsilon \rightarrow 0$.

Proof. Differentiating the identity

$$
\dot{\eta}(t, c) \equiv f(t, \eta(t, c))
$$

with respect to $c$ and then putting $c=\varrho_{0}(\gamma)$ we find easily that $\boldsymbol{\eta}_{c}\left(t, \varrho_{0}(\gamma)\right)$ is a fundamental matrix of solutions of (3.14) for $\gamma \in \mathcal{G}$. Hence by (III), (Ib), (IId) and Theorem 1.4

$$
\chi\left(\frac{\mathbf{D u}}{\mathbf{D} \boldsymbol{c}}\right)=\chi\left(\frac{\mathbf{D} \tilde{u}}{\mathbf{D} \mathbf{x}_{a}} \frac{\mathbf{D}_{a}}{\mathbf{D} \mathbf{c}}+\frac{\mathbf{D} \tilde{u}}{\mathbf{D} \mathbf{x}_{b}} \frac{\mathbf{D} \tilde{\boldsymbol{\eta}}_{b}}{\mathbf{D} \mathbf{c}}\right)=n-k \text { for } \gamma \in \mathbb{F} .
$$

Thus there exist sets $\mathscr{S} \subset \mathscr{N}, \mathscr{V}_{1} \subset \mathscr{N}, v(\mathscr{S})=v\left(\mathscr{V}_{1}\right)=k$ and $\mathscr{S}_{0}, \mathscr{G}_{0}$ a domain in (3) such that

$$
\operatorname{det}\left(\frac{\mathbf{D} \tilde{u}}{\mathbf{D} \mathbf{c}}\right)_{\overline{\mathscr{S}}_{\bar{r}}} \neq 0 .
$$

Let us show that $\mathscr{V}=\mathscr{V}_{1}$ may be put. In fact, differentiating the identity

$$
\mathbf{u}_{\overline{\mathcal{S}}}\left(\boldsymbol{\eta}\left(a, \varrho_{0}(\gamma)\right), \boldsymbol{\eta}\left(b, \varrho_{0}(\gamma)\right)\right) \equiv 0
$$


with respect to $\gamma$, we get

$$
\left(\frac{\mathbf{D} \tilde{u}}{\mathbf{D c}}\right)_{\overline{\mathscr{S}} \overline{\mathscr{V}}} \frac{\mathbf{D} \sigma_{0}}{\mathbf{D} \gamma}+\left(\frac{\mathbf{D} \tilde{u}}{\mathbf{D c}}\right)_{\overline{\mathscr{S}} \boldsymbol{\gamma}} \equiv 0
$$

Let us suppose that

$$
\chi\left(\frac{\mathbf{D} \tilde{u}}{\mathbf{D c}}\right)_{\overline{\mathscr{S}} \overline{\mathcal{V}}} \leqq n-k-1 \text { for some } \stackrel{\circ}{\gamma} \in \mathfrak{S}_{0} .
$$

Then there exist an index $j \in \overline{\mathscr{S}}$ and a $1 \times(n-k-1)$ matrix $\boldsymbol{M}$ such that (denoting $\mathscr{J}$ the complement of $j$ with respect to $\overline{\mathscr{S}}$ )

$$
\left(\frac{\mathbf{D} \tilde{u}}{\mathbf{D c}}\right)_{j, \overline{\mathscr{V}}}-\mathbf{M}\left(\frac{\mathbf{D} \tilde{u}}{\mathbf{D c}}\right)_{\mathscr{J} \overline{\mathscr{V}}}=0 \text { for } \gamma=\stackrel{\circ}{\gamma} .
$$

Then by (3.23)

But then evidently

$$
\left(\frac{\mathbf{D u}}{\mathbf{D c}}\right)_{j, \mathscr{V}}-\mathbf{M}\left(\frac{\mathbf{D u}}{\mathbf{D c}}\right)_{\mathscr{J} \mathscr{V}}=0 \text { for } \gamma=\dot{\gamma} .
$$

$$
\chi\left(\frac{\mathbf{D} \tilde{u}}{\mathbf{D c}}\right)_{\overrightarrow{\mathcal{S}}} \leqq n-k-1 \text { for } \gamma=\stackrel{\circ}{\gamma}
$$

which contradicts $\left(3.22^{\prime}\right)$.

According to $(3.22)$ and $\left(3.22^{\prime}\right)$ there exists a $k \times(n-k)$ matrix $T(\gamma)$ such that

$$
\left(\frac{D \tilde{u}}{D c}\right)_{\mathscr{S}} \equiv T(\gamma)\left(\frac{D \tilde{u}}{D c}\right)_{\overline{\mathscr{S}}}
$$

which in particular yields

$$
\left(\frac{D \tilde{u}}{D c}\right)_{\mathscr{S} \overline{\mathscr{V}}}-T(\gamma)\left(\frac{D \tilde{u}}{D c}\right)_{\overline{\mathscr{S}} \overline{\mathscr{V}}} \equiv 0,
$$

and

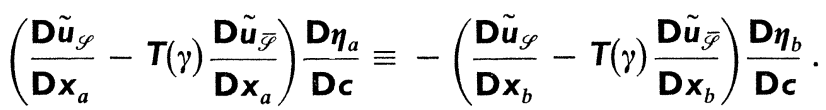

Let us now show that there exists the matrix $\Psi(t, \gamma)$ defined in (III) and namely that

$$
\Psi(t, \gamma) \equiv L(t, \gamma)
$$

where

$$
\mathbf{L}(t, \gamma) \equiv\left(\frac{\mathbf{D} \tilde{\mathbf{u}}_{\mathscr{S}}}{\mathbf{D} \boldsymbol{x}_{\boldsymbol{b}}}-\mathbf{T}(\gamma) \frac{\mathbf{D} \tilde{\mathbf{u}}_{\tilde{\mathcal{F}}}}{\mathbf{D} \mathbf{x}_{\boldsymbol{b}}}\right) \frac{\mathbf{D} \tilde{\boldsymbol{\eta}}_{b}}{\mathbf{D c}}\left(\frac{\mathbf{D} \tilde{\boldsymbol{\eta}}}{\mathbf{D} \boldsymbol{c}}(t)\right)^{-1} .
$$

In fact we may find a system of complementary boundary conditions

$$
\tilde{\mathbf{M}}(\gamma) \mathbf{z}(a)+\tilde{\mathbf{N}}(\gamma) \mathbf{z}(b)=0
$$


with $n \times n$ matrices $\tilde{\mathbf{M}}(\gamma), \tilde{\mathbf{N}}(\gamma) \in C^{1}$ for $\gamma \in \mathscr{S}$ such that

$$
\chi\left(\begin{array}{ll}
\mathbf{M}(\gamma) & \mathbf{N}(\gamma) \\
\tilde{\mathbf{M}}(\gamma) & \tilde{\mathbf{N}}(\gamma)
\end{array}\right)=2 n, \quad\left(\boldsymbol{M}(\gamma)=\frac{\mathbf{D} \tilde{u}}{\mathbf{D} \mathbf{x}_{a}}, \mathbf{N}(\gamma)=\frac{\mathbf{D} \tilde{u}}{\mathbf{D} \mathbf{x}_{b}}\right),
$$

for $\gamma=\mathfrak{G}_{1}, \mathfrak{G}_{1}$ a domain in $\mathfrak{G}_{0}$. Then by Remark 1.1 and Theorem 1.3 there exist $n \times n$ matrices $\boldsymbol{P}(\gamma), \mathbf{Q}(\gamma) \in C^{1}$ for $\gamma \in \mathfrak{G}_{1}$ such that relations (3.19) are fulfilled. Thus the boundary value problem $\left(\lambda^{*}\right)$ is defined for $\gamma \in \mathscr{G}_{1}$ and by Theorems 1.3 and 1.5 it has exactly $k$ linearly independent solutions for $\gamma \in \mathfrak{G}_{1}$. Since $\boldsymbol{\eta}_{c}^{-1}\left(t, \varrho_{0}(\gamma)\right)$ represents a fundamental matrix of solutions of (3.17), rows of the matrix $L(t, \gamma)$ form $k$ solutions of these equations. Further the matrix $L(t, \gamma)$ obviously fulfils the condition (3.20). Finally substituting $L(t, \gamma)$ into (3.18) and making use of (3.24") and (3.19) we get

$$
\begin{aligned}
& \mathbf{L}(a, \gamma) \mathbf{P}^{\prime}(\gamma)+\mathbf{L}(b, \gamma) \mathbf{Q}^{\prime}(\gamma) \equiv \\
& \equiv\left(\mathbf{N}_{\mathscr{S}}(\gamma)-\boldsymbol{T}(\gamma) \mathbf{N}_{\overline{\mathcal{Y}}}(\gamma)\right) \tilde{\boldsymbol{\eta}}_{\boldsymbol{c}}(b) \boldsymbol{\eta}_{\mathbf{c}}^{-1}(a) \boldsymbol{P}^{\prime}(\gamma)+ \\
& +\left(\boldsymbol{N}_{\mathscr{S}}(\gamma)-\mathbf{T}(\gamma) \mathbf{N}_{\overline{\mathscr{S}}}(\gamma)\right) \cdot \boldsymbol{\eta}_{\mathbf{c}}(b) \boldsymbol{\eta}_{c}^{-1}(b) \mathbf{Q}^{\prime}(\gamma)= \\
& =-\left(\boldsymbol{M}_{\mathscr{S}}(\gamma)-\boldsymbol{T}(\gamma) \boldsymbol{M}_{\overline{\mathscr{S}}}(\gamma)\right) \boldsymbol{\eta}_{\boldsymbol{c}}(a) \boldsymbol{\eta}_{\boldsymbol{c}}^{-1}(a) \boldsymbol{P}^{\prime}(\gamma)+\left(\boldsymbol{N}_{\mathscr{S}}(\gamma)-\boldsymbol{T}(\gamma) \boldsymbol{N}_{\overline{\mathscr{S}}}(\gamma)\right) \mathbf{Q}^{\prime}(\gamma) \equiv 0
\end{aligned}
$$

which completes the proof of (3.25). (From (Ib), (IId) and (3.24") it follows readily that the rows of $\boldsymbol{\Psi}(t, \gamma)$ are linearly independent.)

The matrices $\boldsymbol{T}(\gamma)$ and $\boldsymbol{\Psi}(t, \gamma)$ having been determined, the equations (3.21) are completely defined and their solution $\gamma_{0}=\gamma_{0}^{*}$ with properties (IVb, c) may be found.

Now by (V), Theorem 2.1 and the theorem on continuous dependence of solutions on initial values and parameters there exists a neighborhood $\mathfrak{D}_{1}\left(c_{0}^{*}, \delta_{1}\right), c_{0}^{*}=\varrho_{0}\left(\gamma_{0}^{*}\right)$ and an interval $\xi_{1}=\left\langle 0, \varepsilon_{1}\right\rangle, 0<\varepsilon_{1} \leqq \varepsilon_{0}$ such that (1) every solution $\xi(t, \boldsymbol{c}, \varepsilon)$ of (3.1), where $\varepsilon \in \mathfrak{F}_{1}$, with $\xi(a, c, \varepsilon)=c$ stays in $\mathfrak{X}$ for $t \in \mathfrak{I}$ and it may be written in the form

$$
\xi(t, c, \varepsilon)=\boldsymbol{\eta}(t, \boldsymbol{C}(t, c, \varepsilon))
$$

where $\mathbf{C}(t, \boldsymbol{c}, \varepsilon)$ is the solution of equations

$$
\boldsymbol{\Gamma}(t)=\mathbf{c}+\varepsilon \int_{\boldsymbol{a}}^{t} \boldsymbol{\eta}_{\boldsymbol{c}}^{-1}(s, \boldsymbol{\Gamma}(s)) \mathbf{g}(s, \boldsymbol{\eta}(s, \boldsymbol{\Gamma}(s)), \varepsilon) \mathrm{d} s
$$

and (2) according to $\left(3.22^{\prime}\right)$ where $\mathscr{V}_{1}=\mathscr{V}$,

$$
\operatorname{det}\left(\frac{\mathbf{D w}}{\mathbf{D} c}\left(\xi_{a}, \xi_{b}, \varepsilon\right)\right)_{\overline{\mathcal{S}} \overline{\mathcal{V}}} \neq 0 .
$$

A solution $\xi(t, \boldsymbol{c}, \varepsilon)$ of (3.1) is a solution of the boundary value problem $(v)$ if and only if

$$
\mathbf{w}\left(\xi_{a}, \xi_{b}, \varepsilon\right)=0 .
$$

This is a system of $n$ equations for $n$ components of the initial vector c. Putting 
$\varepsilon=0$ in (3.30) we get a system of $n$ necessary conditions

$$
\boldsymbol{u}\left(\boldsymbol{\eta}\left(a, \boldsymbol{c}_{0}\right), \boldsymbol{\eta}\left(b, \boldsymbol{c}_{0}\right)\right)=0
$$

which $n$ components of the vector $c_{0}=c(0)$ have to fulfil. This system is identical (with the exception of notation) with the system (3.4) about which we know that it has not a uniquely determined solution. Thus the system (3.30) is not suitable in this form to investigate the existence of its solution by means of the implicit function theorem. Therefore we shall modify it in the following way.

Leaving equations (3.30) with indices from $\overline{\mathscr{S}}$ unchanged, we have

$$
\mathbf{w}_{\overline{\mathscr{S}}}\left(\boldsymbol{\xi}_{a}, \boldsymbol{\xi}_{b}, \varepsilon\right)=0 \text {. }
$$

By (3.29) and the implicit function theorem there exist a neigborhood $\mathfrak{N}_{2}\left(\gamma_{0}^{*}, \delta_{2}\right) \subset \mathfrak{G}_{1}$, $0<\delta_{2} \leqq \delta_{1}$ and an interval $\mathfrak{E}_{2}=\left\langle 0, \varepsilon_{2}\right\rangle, 0<\varepsilon_{2} \leqq \varepsilon_{1}$ such that for $\boldsymbol{c}_{\mathscr{\gamma}} \in \mathfrak{N}_{2}\left(\gamma_{0}^{*}, \delta_{2}\right)$ and $\varepsilon \in \mathcal{F}_{2}$ there exists a solution

$$
c_{\bar{V}}=\sigma\left(c_{\mathscr{V}}, \varepsilon\right)
$$

of (3.30) such that (denoting again $c_{\gamma}=\gamma, \varrho(\gamma, \varepsilon)=\gamma+\sigma(\gamma, \varepsilon)$ )

$$
\sigma(\gamma, \varepsilon) \in C^{2,1}, \sigma(\gamma, 0)=\sigma_{0}(\gamma) \text { and } \varrho(\gamma, \varepsilon) \in \mathfrak{D}_{1}\left(c_{0}^{*}, \delta_{1}\right) .
$$

Inserting (3.32) into (3.31), denoting

$$
\mathfrak{g}(\gamma, \varepsilon)=\int_{a}^{b} \boldsymbol{\eta}_{c}^{-1}(s, \boldsymbol{C}(s, \varrho(\gamma, \varepsilon), \varepsilon)) \boldsymbol{g}(s, \boldsymbol{\eta}(s, \boldsymbol{C}(s, \varrho(\gamma, \varepsilon)), \varepsilon) \mathrm{d} s
$$

and making use of the mean value theorem we get the identity

$$
\begin{aligned}
& \mathbf{w}_{\overline{\mathcal{A}}}\left(\tilde{\xi}_{a}, \tilde{\boldsymbol{\xi}}_{b}, \varepsilon\right) \equiv \boldsymbol{u}_{\overline{\mathcal{S}}}\left(\tilde{\boldsymbol{\eta}}_{\boldsymbol{a}}, \tilde{\boldsymbol{\eta}}_{b}\right)+\varepsilon \int_{0}^{1}\left[\left(\frac{\mathbf{D} \tilde{\boldsymbol{u}}}{\mathbf{D} \boldsymbol{c}}\right)_{\overline{\mathcal{P}} \tilde{\mathcal{V}}} \frac{\partial \boldsymbol{\sigma}}{\partial \varepsilon}\right]_{\vartheta} \mathrm{d} \vartheta+ \\
& +\varepsilon \int_{0}^{1}\left[\frac{\mathbf{D} \tilde{\mathbf{u}}_{\mathscr{S}}}{\mathbf{D} \boldsymbol{x}_{b}} \frac{\mathbf{D} \boldsymbol{\eta}_{b}}{\mathbf{D} \boldsymbol{c}}\right]_{\vartheta} \mathrm{d} \vartheta \mathfrak{g}(\gamma, \varepsilon)+\varepsilon \boldsymbol{v}_{\overline{\mathcal{F}}}\left(\tilde{\xi}_{a}, \tilde{\xi}_{b}, \varepsilon\right) \equiv 0,
\end{aligned}
$$

where $\tilde{\xi}(t)=\xi(t, \varrho(\gamma, \varepsilon), \varepsilon)$ and the meaning of the index $\vartheta$ may easily be found in each case.

Inserting (3.32) into equations (3.30) with indices from $\mathscr{S}$ we get

$$
\mathbf{w}_{\mathcal{S}}\left(\tilde{\xi}_{a}, \tilde{\boldsymbol{\xi}}_{b}, \varepsilon\right)=0
$$

or making use again of the mean value theorem

$$
\begin{aligned}
& \mathbf{w}_{\mathscr{S}}\left(\tilde{\xi}_{a}, \tilde{\boldsymbol{\xi}}_{b}, \varepsilon\right) \equiv \boldsymbol{u}_{\mathscr{S}}\left(\tilde{\boldsymbol{\eta}}_{a}, \tilde{\boldsymbol{\eta}}_{b}\right)+\varepsilon \int_{0}^{1}\left[\left(\frac{\mathbf{D} \tilde{u}}{\mathbf{D c}_{\boldsymbol{c}}}\right)_{\mathscr{\mathscr { S }} \boldsymbol{\mathcal { V }}} \frac{\partial \boldsymbol{\sigma}}{\partial \varepsilon}\right]_{\vartheta} \mathrm{d} \vartheta+ \\
& +\varepsilon \int_{0}^{1}\left[\frac{\mathbf{D} \tilde{\boldsymbol{u}}_{\mathscr{c}}}{\mathbf{D} \boldsymbol{x}_{b}} \frac{\mathbf{D} \tilde{\boldsymbol{\eta}}_{b}}{\mathbf{D c}}\right]_{\vartheta} \mathrm{d} \vartheta \mathfrak{g}(\gamma, \varepsilon)+\varepsilon \mathbf{v}_{\mathcal{S}}\left(\tilde{\xi}_{a}, \tilde{\xi}_{b}, \varepsilon\right)=0 .
\end{aligned}
$$

By (3.34) the system of equations $\left(3.35^{\prime}\right)$ may be replaced for $\varepsilon \neq 0$ by an equivalent system of equations

$$
\mathfrak{w}(\gamma, \varepsilon) \equiv \frac{1}{\varepsilon}\left[\mathbf{w}_{\mathcal{S}}\left(\tilde{\xi}_{a}, \tilde{\xi}_{b}, \varepsilon\right)-\boldsymbol{T}(\gamma) \mathbf{w}_{\tilde{\mathcal{S}}}\left(\tilde{\xi}_{a}, \tilde{\xi}_{b}, \varepsilon\right)\right]=0 .
$$


Now letting $\varepsilon \rightarrow 0$ in (3.36) and taking into account (IIb), (3.24), (3.24'), (3.25) and (3.26) we get the system

$$
\mathfrak{w}_{0}\left(\gamma_{0}\right) \equiv \int_{a}^{b} \Psi\left(s, \gamma_{0}\right) \mathbf{g}(s, \tilde{\boldsymbol{\eta}}(s), 0) \mathrm{d} s+\tilde{\boldsymbol{v}}_{0 \mathscr{S}}-\boldsymbol{T}\left(\gamma_{0}\right) \tilde{\boldsymbol{v}}_{0 \overline{\mathscr{T}}}=0,
$$

where $\tilde{\boldsymbol{v}}_{0}=\mathbf{v}\left(\tilde{\boldsymbol{\eta}}_{a}, \tilde{\boldsymbol{\eta}}_{b}, 0\right)$, which is identical with the system (3.21). By assumptions (I), (II), (IV) and previous considerations $\mathfrak{w}(\gamma, \varepsilon) \in C^{\mathbf{1}, 1}$ for $\gamma \in \mathfrak{N}_{2}\left(\gamma_{0}^{*}, \delta_{2}\right)$ and $\varepsilon \in\left(0, \varepsilon_{2}\right)$. Putting $\mathfrak{w}(\gamma, 0)=\mathfrak{w}_{0}(\gamma)$ we may verify easily that $\lim _{e \rightarrow 0}(\mathfrak{w}(\gamma, \varepsilon)-\mathfrak{w}(\gamma)) / \varepsilon$ exists; thus $\mathfrak{w}(\gamma, \varepsilon) \in C^{1,1}$ on $\mathfrak{N}_{2}\left(\gamma_{0}^{*}, \delta_{2}\right) \times \mathfrak{F}_{2}$. Hence by (IVa, b, c) equations (3.36) satisfy all assumptions of the implicit function theorem and by it we may conclude that there exists a unique solution $\gamma=\gamma^{*}(\varepsilon) \in C^{1}$ of (3.36) for sufficiently small $\varepsilon>0$ with $\gamma^{*}(0)=\gamma_{0}^{*}$. Then $c_{\bar{\gamma}}=\sigma\left(\gamma^{*}(\varepsilon), \varepsilon\right)$ is a unique solution of $(3.31)$ with $\sigma\left(\gamma^{*}(0), 0\right)=$ $=\sigma_{0}\left(\gamma_{0}^{*}\right)$ and $c=\varrho\left(\gamma^{*}(\varepsilon), \varepsilon\right)$ is a unique solution of (3.30) with $\varrho\left(\gamma^{*}(0), 0\right)=c_{0}^{*}$ which completes the proof of Theorem 3.2.

Corollary 3.3. Let an interval $\mathfrak{I}=\langle a, b\rangle$ be given. Consider the boundary value problem $(\kappa)$ given by (3.10), (3.11). Let the following assumptions be fulfilled.

(I) Let $\chi(\mathbf{M}: \mathbf{N})=n$. Let $\mathbf{v}(\mathbf{p}, \boldsymbol{q}, \varepsilon) \in C^{1,1,1}$ for $\boldsymbol{p} \in \mathfrak{P}, \boldsymbol{q} \in \mathfrak{D}, \varepsilon \in(F$ where $\mathfrak{P}$ and $\mathfrak{D}$ are open sets in $E^{n}$ and $\left.\varepsilon \in \mathcal{F}=\left\langle 0, \varepsilon_{0}\right\rangle, \varepsilon_{0}\right\rangle 0$.

(II) Let the shortened boundary value problem $\left(\kappa_{0}\right)$ given by (3.12) have exactly $k$ linearly independent solutions. Denote $\tilde{\boldsymbol{\Phi}}(t) a n \times k$ matrix whose columns are formed by these solutions.

(III) There exist a set $\mathscr{S} \subset \mathscr{N}, v(\mathscr{S})=k$ and a constant $k \times(n-k)$ matrix $T$ such that for any fundamental matrix $\Phi(t)$ of solutions of $\left(3.12_{1}\right)$ it holds

$$
(\mathbf{M} \boldsymbol{\Phi}(a)+\mathbf{N} \boldsymbol{\Phi}(b))_{\mathscr{S}} \equiv \boldsymbol{T}(\mathbf{M} \boldsymbol{\Phi}(a)+\mathbf{N} \boldsymbol{\Phi}(b))_{\overrightarrow{\mathscr{S}}} .
$$

Further there exists a $k \times n$ matrix $\Psi(t)$ whose rows are formed by solutions of the boundary value problem $\left(\kappa_{0}^{*}\right)$ (adjoint to the shortened boundary value problem $\left.\left(\kappa_{0}\right)\right)$

$$
\dot{w}^{\prime}=-\mathbf{w}^{\prime} \mathbf{A}(t), \quad \mathbf{w}^{\prime}(a) \mathbf{P}^{\prime}+\mathbf{w}^{\prime}(b) \mathbf{Q}^{\prime}=0,
$$

where $-\mathbf{M} \mathbf{P}^{\prime}+\mathbf{N} \mathbf{Q}^{\prime}=0, \chi(\mathbf{P}: \mathbf{Q})=n$, and which fulfils the condition

$$
\boldsymbol{\Psi}(b)=\boldsymbol{N}_{\mathscr{S}}-\mathbf{T} \boldsymbol{N}_{\overline{\mathscr{S}}} .
$$

(a) Let equations

$$
\int_{a}^{b} \boldsymbol{\Psi}(s) \boldsymbol{g}\left(s, \tilde{\boldsymbol{\Phi}}(s) \gamma_{0}, 0\right) \mathrm{d} s+\mathbf{v}_{0 \mathscr{S}}-\boldsymbol{T} \mathbf{v}_{0 \overline{\mathscr{S}}}=0,
$$

where $\gamma$ is a k-vector and $\mathbf{v}_{0}=\mathbf{v}\left(\tilde{\boldsymbol{\Phi}}(a) \gamma_{0}, \tilde{\boldsymbol{\Phi}}(b) \gamma_{0}, 0\right)$, have a real solution $\gamma_{0}=\gamma_{0}^{*}$.

(b) Let $\tilde{\boldsymbol{\Phi}}(a) \gamma_{0}^{*} \in \mathfrak{P}, \tilde{\boldsymbol{\Phi}}(b) \gamma_{0}^{*} \in \mathbf{Q}$.

(c) Let the jacobian of the system (3.38) with respect to $\gamma_{0}$ be nonvanishing at the point $\gamma_{0}=\gamma_{0}^{*}$. 
(IV) Denote $\mathfrak{X}=E\left[\mathbf{x} \mid\left\|\mathbf{x}-\tilde{\boldsymbol{\Phi}}(t) \gamma_{0}^{*}\right\|<\varrho, t \in \mathfrak{T}\right], \varrho>0$. Let $\mathbf{A}(t) \in C^{0}, \mathbf{g}(t, \mathbf{x}, \varepsilon) \in$ $\in C^{0,1,1}$ for $t \in \mathfrak{Z}, \boldsymbol{x} \in \mathfrak{X}, \varepsilon \in \mathfrak{E}$.

Then there exists for sufficiently small $\varepsilon>0$ a unique solution $\xi\left(t, c^{*}(\varepsilon), \varepsilon\right)$ of the problem $(\kappa)$ with $\xi\left(a, c^{*}(\varepsilon), \varepsilon\right)=c^{*}(\varepsilon) \in C^{1}$ which tends to the solution $\tilde{\boldsymbol{\Phi}}(t) \gamma_{0}^{*}$ of the shortened boundary value problem $\left(\kappa_{0}\right)$ as $\varepsilon \rightarrow 0$.

Corollary 3.4. Consider the differential system (3.1). Let the following assumptions be fulfilled.

(I) Let $\mathbf{f}(t, \mathbf{x})$ and $\mathbf{g}(t, \mathbf{x}, \varepsilon)$ be $\omega$-periodic in $t$.

(II) (a) (b) (c) The same as in Theorem 3.2 with the exception that $a=0, b=\omega$ and that $\mathbf{u}\left(\boldsymbol{\eta}_{0}, \boldsymbol{\eta}_{\omega}\right)$ has the particular form $\mathbf{u}\left(\boldsymbol{\eta}_{0}, \boldsymbol{\eta}_{\omega}\right)=\boldsymbol{\eta}_{0}-\boldsymbol{\eta}_{\omega}$.

(III) Let the system

$$
\dot{\mathbf{z}}=f_{\mathbf{x}}(t, \tilde{\boldsymbol{\eta}}(t)) \mathbf{z}
$$

have exactly $k$ linearly independent $\omega$-periodic solutions for $\gamma \in \mathfrak{G}$.

Then the adjoint differential system

$$
\dot{\boldsymbol{w}}^{\prime}=-\boldsymbol{w}^{\prime} \boldsymbol{f}_{\mathbf{x}}(t, \tilde{\boldsymbol{\eta}}(t))
$$

has also exactly $k$ linearly independent solutions for $\gamma \in \mathfrak{G}$. Denote $\Psi(t, \gamma)$ a $k \times n$ matrix whose rows are formed by them.

(IV) (a) Let the system

$$
\int_{0}^{\omega} \boldsymbol{\Psi}\left(s, \gamma_{0}\right) \mathbf{g}(s, \tilde{\boldsymbol{\eta}}(s), 0) \mathrm{d} s=0
$$

have a real solution $\gamma_{0}=\gamma_{0}^{*} \in \mathbb{B}$.

(b) Let the jacobian of the system (3.39) with respect to $\gamma_{0}$ be nonvanishing at the point $\gamma_{0}=\gamma_{0}^{*}$.

(V) The same as in Theorem 3.2 with the exception that $\mathfrak{Z}=\mathfrak{I}_{\omega}=\langle 0, \omega\rangle$.

Then there exists for sufficiently small $\varepsilon>0$ a unique $\omega$-periodic solution $\xi\left(t, c^{*}(\varepsilon), \varepsilon\right)$ of (3.1) with $\xi\left(a, \mathbf{c}^{*}(\varepsilon), \varepsilon\right)=\mathbf{c}^{*}(\varepsilon) \in C^{1}$ which tends to the $\omega$-periodic solution $\boldsymbol{\eta}\left(t, \varrho_{0}\left(\gamma_{0}^{*}\right)\right)$ of $(3.3)$ as $\varepsilon \rightarrow 0$.

Remark 3.1. Clearly we may choose instead of the vector $c$ some other parameters by which the solution of (3.1) is uniquelly determined. (We have made use of this fact in Corollary 3.3.)

Remark 3.2. It may happen that it is convenient or necessary to suppose that the length of the interval on which we consider the perturbed boundary value problem $(v)$ is also a function of $\varepsilon$. Let us put $b(\varepsilon)=b+\tau(\varepsilon)$ where $b$ is the right-hand endpoint of the interval $\langle a, b\rangle$ on which the shortened problem $\left(v_{0}\right)$ is considered and $\tau(\varepsilon) \in C^{1}$ for $\varepsilon \in(E$ with $\tau(0)=0$. It is obvious from proofs of Theorems 3.1 and 3.2 that in both cases the existence of a solution of the problem $(v)$ may be proved when we substitute $\mathfrak{I}_{\varepsilon}=\langle a, b+\tau(\varepsilon)\rangle$ instead of $\mathfrak{Z}$ and leave the other assumptions unchanged. 
Remark 3.3. The solution of the boundary value problem $(v)$ (be it under the assumptions of Theorem 3.1 or Theorem 3.2) may be found by means of the method of successive approximations. (See [6].)

If the functions $\boldsymbol{f}(t, \boldsymbol{x})$ and $\mathbf{g}(t, \mathbf{x}, \varepsilon)$ are analytic in $\mathbf{x}$ and $\varepsilon$ and the functions $\mathbf{u}(\boldsymbol{p}, \boldsymbol{q})$ and $\mathbf{v}(\boldsymbol{p}, \boldsymbol{q}, \varepsilon)$ are analytic in $\boldsymbol{p}, \boldsymbol{q}$ and $\varepsilon$ on sets given in Theorems 3.1 or 3.2 , it may be shown that the found solution is analytic in $\varepsilon$ (for sufficiently small $\varepsilon>0$ ) and it may be obtained by solving recursively a sequence of boundary value problems (from which only one is nonlinear).

Remark 3.4. A system of necessary conditions (equivalent with (3.21)), which the vector $\gamma$ has to fulfil, may be found by a somewhat different consideration than that presented in the main text.

Under the assumptions of Theorem 3.2 every solution $\xi(t, c+\varepsilon d(\varepsilon), \varepsilon)$ with $\xi(a, c+\varepsilon \boldsymbol{d}(\varepsilon), \varepsilon)=\boldsymbol{c}+\varepsilon \boldsymbol{d}(\varepsilon)$ of the system (3.1) may be written in the form $\boldsymbol{\xi}(t, \boldsymbol{c}+\varepsilon \boldsymbol{d}, \varepsilon)=\boldsymbol{\eta}(t, \boldsymbol{c})+\varepsilon \zeta(t, \boldsymbol{c}, \boldsymbol{d}, \varepsilon)$ where $\boldsymbol{\eta}(t, \boldsymbol{c}) \in C^{1,2}, \zeta(t, \boldsymbol{c}, \boldsymbol{d}, \varepsilon) \in C^{1,1,1,1}$ and $\boldsymbol{\eta}(a, \boldsymbol{c})=\boldsymbol{c}, \zeta(a, \boldsymbol{c}, \boldsymbol{d}, \varepsilon)=\boldsymbol{d}$. If $\boldsymbol{\xi}(t, \boldsymbol{c}+\varepsilon \boldsymbol{d}, \varepsilon)$ has to be a solution of the boundary value problem $(v)$ then the functions $\boldsymbol{\eta}(t, \boldsymbol{c})$ and $\zeta(t, \boldsymbol{c}, \boldsymbol{d}, \varepsilon)$ have to fulfil boundary value problems

$$
\dot{\boldsymbol{\eta}}=\boldsymbol{f}(t, \boldsymbol{\eta}), \quad \boldsymbol{u}(\boldsymbol{\eta}(a), \boldsymbol{\eta}(b))=0,
$$

and

$$
\dot{\zeta}=\int_{0}^{1} \frac{\mathbf{D f}}{\mathbf{D x}}(t, \boldsymbol{\eta}+\vartheta \varepsilon \zeta) \mathrm{d} \vartheta \zeta+\boldsymbol{g}(t, \boldsymbol{\eta}+\varepsilon \zeta, \varepsilon),
$$

$$
\begin{gathered}
\int_{0}^{1} \frac{\mathbf{D u}}{\mathbf{D} \boldsymbol{x}_{a}}\left(\boldsymbol{\eta}_{a}+\vartheta \varepsilon \zeta_{a}, \boldsymbol{\eta}_{b}+\vartheta \varepsilon \zeta_{b}\right) \mathrm{d} \vartheta \zeta(a)+\int_{0}^{1} \frac{\mathbf{D u}}{\mathbf{D x}}\left(\boldsymbol{\eta}_{a}+\vartheta \varepsilon \zeta_{a}, \boldsymbol{\eta}_{b}+\vartheta \varepsilon \zeta_{b}\right) \mathrm{d} \vartheta \zeta(b)+ \\
+\mathbf{v}\left(\xi_{a}, \boldsymbol{\xi}_{b}, \varepsilon\right)=0
\end{gathered}
$$

respectively.

The boundary value problem (3.40) has the $k$-parametric solution $\tilde{\boldsymbol{\eta}}(t)=\boldsymbol{\eta}\left(t, \varrho_{0}(\gamma)\right)$. Letting $\varepsilon \rightarrow 0$ in (3.41) and then substituting $\tilde{\boldsymbol{\eta}}(t)$ for $\boldsymbol{\eta}$, we get the boundary value problem

$$
\begin{gathered}
\dot{\zeta}_{0}=\boldsymbol{f}_{\boldsymbol{x}}\left(t, \tilde{\boldsymbol{\eta}}(t) \zeta_{0}+\mathbf{g}(t, \tilde{\boldsymbol{\eta}}(t), 0),\right. \\
\frac{\mathrm{Du}}{\mathbf{D} \boldsymbol{x}_{\boldsymbol{a}}}\left(\tilde{\boldsymbol{\eta}}_{a}, \tilde{\boldsymbol{\eta}}_{b}\right) \zeta_{0}(a)+\frac{\mathbf{D u}}{\mathbf{D} \boldsymbol{x}_{\boldsymbol{b}}}\left(\tilde{\boldsymbol{\eta}}_{a}, \tilde{\boldsymbol{\eta}}_{b}\right) \zeta_{0}(b)+\mathbf{v}\left(\tilde{\boldsymbol{\eta}}_{a}, \tilde{\boldsymbol{\eta}}_{b}, 0\right)=0 .
\end{gathered}
$$

The homogeneous boundary value problem associated to this problem is evidently the problem $(\lambda)$ given by (3.14), (3.15). Now by Theorem 1.5 the problem (3.42) has a solution if and only if

$$
\int_{a}^{b} \Psi_{0}(t, \gamma) \mathbf{g}(t, \tilde{\boldsymbol{\eta}}(t), 0) \mathrm{d} t+\left(\Psi_{0}(a, \gamma) \tilde{\boldsymbol{P}}^{\prime}(\gamma)+\Psi_{0}(b, \gamma) \tilde{\mathbf{Q}}^{\prime}(\gamma)\right) \mathbf{v}\left(\tilde{\boldsymbol{\eta}}_{\boldsymbol{a}}, \tilde{\boldsymbol{\eta}}_{b}, 0\right)=0
$$

where rows of the $k \times n$ matrix $\Psi_{0}(t, \gamma)$ are formed by some $k$ linearly independent solutions of the problem $\left(\lambda^{*}\right)$ (given by (3.17), (3.18)) and the $n \times 2 n$ matrix $(\tilde{\boldsymbol{P}}(\gamma)$ : 
: $\tilde{\mathbf{Q}}(\gamma))$ defines the complementary adjoint boundary conditions to boundary conditions (3.15) for $\gamma \in \mathfrak{G H}_{1}$.

It may be easily verified that, if $\Psi_{0}(t, \gamma)$ satisfies the condition (3.20) (so that $\left.\Psi_{0}(t, \gamma) \equiv \Psi(t, \gamma)\right)$, the condition (3.43) reduces to (3.21).

Indeed, by (3.25), (3.26) and (3.24")

$$
\begin{aligned}
& \boldsymbol{\Psi}_{0}(a, \gamma) \tilde{\mathbf{P}}^{\prime}(\gamma)+\boldsymbol{\Psi}_{0}(b, \gamma) \tilde{\mathbf{Q}}^{\prime}(\gamma) \equiv \\
& \equiv-\left(\frac{\mathbf{D} \tilde{\mathbf{u}}_{\mathscr{f}}}{\mathbf{D} \boldsymbol{x}_{a}}-\mathbf{T}(\gamma) \frac{\mathbf{D} \tilde{\boldsymbol{u}}_{\tilde{\varphi}}}{\mathbf{D} \boldsymbol{x}_{a}}\right) \tilde{\boldsymbol{P}}^{\prime}(\gamma)+\left(\frac{\mathbf{D} \mathbf{u}_{\mathscr{\varphi}}}{\mathbf{D} \boldsymbol{x}_{b}}-\mathbf{T}(\gamma) \frac{\mathbf{D} \mathbf{u}_{\bar{f}}}{\mathbf{D} \mathbf{x}_{b}}\right) \tilde{\mathbf{Q}}^{\prime}(\gamma) \text {. }
\end{aligned}
$$

Since by Remark 1.1

so that

$$
-\frac{\mathrm{D} \tilde{u}}{\mathrm{D} \mathbf{x}_{a}} \tilde{\mathbf{P}}(\gamma)+\frac{\mathrm{D} \tilde{u}}{\mathrm{D} \mathbf{x}_{b}} \tilde{\mathbf{Q}}(\gamma) \equiv E_{n},
$$

$$
-\frac{\mathbf{D} \tilde{\mathbf{u}}_{\mathscr{Y}}}{\mathbf{D} \boldsymbol{x}_{a}} \tilde{\mathbf{P}}^{\prime}(\gamma)+\frac{\mathbf{D} \tilde{\mathbf{u}}_{\mathscr{Y}}}{\mathbf{D} \boldsymbol{x}_{a}} \tilde{\mathbf{Q}}^{\prime}(\gamma) \equiv \mathbf{E}_{\mathscr{S}}, \quad-\frac{\mathbf{D} \tilde{\mathbf{u}}_{\overline{\mathscr{Y}}}}{\mathbf{D} \boldsymbol{x}_{b}} \tilde{\mathbf{P}}^{\prime}(\gamma)+\frac{\mathbf{D} \tilde{\mathbf{u}}_{\overline{\mathscr{Y}}}}{\mathbf{D} \boldsymbol{x}_{b}} \tilde{\mathbf{Q}}^{\prime}(\gamma) \equiv \mathbf{E}_{\overline{\mathscr{Y}}},
$$

the equality (3.44) yields

$$
\boldsymbol{\Psi}_{0}(a, \gamma) \tilde{\mathbf{P}}^{\prime}(\gamma)+\boldsymbol{\Psi}_{0}(b, \gamma) \tilde{\mathbf{Q}}^{\prime}(\gamma) \equiv \mathbf{E}_{\mathscr{S}}-\boldsymbol{T}(\gamma) \mathbf{E}_{\overline{\mathscr{S}}}
$$

whence our assertion readily follows.

\section{NONLINEAR BOUNDARY VALUE PROBLEMS FOR AUTONOMOUS DIFFERENTIAL SYSTEMS}

In this section we shall consider the boundary value problem $(\alpha)$ given by

$$
\begin{gathered}
\dot{\boldsymbol{x}}=\boldsymbol{f}(\mathbf{x})+\varepsilon \mathbf{g}(\mathbf{x}, \varepsilon), \\
\mathbf{w}\left(\mathbf{x}_{a}, \boldsymbol{x}_{b}, \varepsilon\right) \equiv \boldsymbol{u}\left(\boldsymbol{x}_{a}, \boldsymbol{x}_{b}\right)+\varepsilon \mathbf{v}\left(\mathbf{x}_{a}, \boldsymbol{x}_{b}, \varepsilon\right)=0 .
\end{gathered}
$$

It is known that this problem with periodic boundary conditions (i. e. $\mathbf{w}\left(\boldsymbol{x}_{a}, \boldsymbol{x}_{b}, \varepsilon\right) \equiv$ $\equiv \boldsymbol{x}_{a}-\boldsymbol{x}_{b}=0$ ) has some characteristic features by which it differs fundamentally from a similar problem with a nonautonomous differential system. Above all it is the fact that one component of the initial vector $c^{*}(\varepsilon)$ of a solution $\xi\left(t, c^{*}(\varepsilon), \varepsilon\right)$ may be chosen arbitrarily (in a certain range) and that the length $l(\varepsilon)=b(\varepsilon)-a$ of the interval on which the solution exists, depends on $\varepsilon$ and has to be taken as a new unknown. Another such fact (closely related to the previous one) is that the length of the interval on which a solution from a $k$-parametric family $\boldsymbol{\eta}(t, \boldsymbol{c}(\gamma))$ of solutions of the shortened boundary value problem $\left(\alpha_{0}\right)$

$$
\begin{gathered}
\dot{y}=f(y), \\
u\left(y_{a}, y_{b}\right)=0
\end{gathered}
$$

exists also depends on the parameters $\gamma$ (we usually choose as these parameters some 
components of the initial vector $\boldsymbol{c}$ ). We shall see later that these features appear in a wider class of boundary value problems with autonomous differential systems (among which however the problems with periodic boundary conditions occupy an outstanding position). That is why we shall suppose from the beginning that the right endpoint $b$ of the interval $\langle a, b\rangle$ on which a solution of the shortened boundary value problem exists is a function of the initial vector c (or of other equivalent parameters) of this solution and that the right endpoint of the interval on which a solution of the given problem $(\alpha)$ exists is a function of the parameter $\varepsilon$.

Let us now investigate the problem $(\alpha)$ in more detail. In the sequel we shall suppose that the following conditions $(\mathscr{C})$ are fulfilled.

Let $\mathfrak{X}$ be a domain in $E^{n}$, let $\mathscr{F}=\left\langle 0, \varepsilon_{0}\right\rangle, \varepsilon_{0}>0$, and let $\mathfrak{I}_{0}=(A, B), A<0<B$. Let $\boldsymbol{f}(\mathbf{x}) \in C^{2}, \boldsymbol{g}(\mathbf{x}, \varepsilon) \in C^{1,0}$ for $\mathbf{x} \in \mathfrak{X}, \cdot \varepsilon \in \mathcal{E}$. Let $\mathfrak{P}$ and $\mathfrak{D}$ be domains in $\mathfrak{X}$ and let $\mathbf{u}(\boldsymbol{p}, \boldsymbol{q}) \in C^{2,2}, \mathbf{v}(\boldsymbol{p}, \boldsymbol{q}, \varepsilon) \in C^{1,1,0}$ for $\boldsymbol{p} \in \mathfrak{P}, \boldsymbol{q} \in \mathfrak{D}, \varepsilon \in \mathfrak{F}$. Let $\mathfrak{E}$ be a domain in $\mathfrak{X}$ and let every solution $\xi(t, \boldsymbol{c}, \varepsilon)$ of $(4.1)$ with $\xi(a, c, \varepsilon)=\boldsymbol{c}$ be defined and stay in $\mathfrak{X}$ for $t \in \mathfrak{I}_{0}, \boldsymbol{c} \in \mathfrak{C}, \varepsilon \in \mathfrak{F}$. Let $b(\varepsilon) \in C^{0}$ and $\mathfrak{I}=\langle a, b(\varepsilon)\rangle \subset \mathfrak{I}_{0}$ for $\varepsilon \in \mathfrak{E}$. Let $\xi(a, \mathbf{c}, \varepsilon) \in \mathfrak{P}$, $\xi(b(\varepsilon), c, \varepsilon) \in \mathbb{D}$ and further let

$$
\chi\left(\frac{\mathbf{D w}}{\mathbf{D x}_{a}}(\boldsymbol{p}, \boldsymbol{q}, \varepsilon): \frac{\mathbf{D w}}{\mathbf{D} \mathbf{x}_{b}}(\boldsymbol{p}, \boldsymbol{q}, \varepsilon)\right)=n
$$

for $\boldsymbol{p} \in \mathfrak{P}, \boldsymbol{q} \in \mathfrak{D}, \varepsilon \in \mathfrak{E}$.

When the dependence on $\varepsilon$ in $(\alpha)$ does not take place at all or when the parameter $\varepsilon$ is fixed, we shall write briefly

$$
\begin{gathered}
\dot{\boldsymbol{x}}=\boldsymbol{h}(\mathbf{x}), \\
\mathbf{z}(\mathbf{x}(a), \mathbf{x}(b))=0
\end{gathered}
$$

and denote it as a problem $\left(\alpha^{\prime}\right)$. In this case we shall suppose that the following conditions $\left(\mathscr{C}^{\prime}\right)$ are fulfilled.

Let $\boldsymbol{h}(\mathbf{x}) \in C^{2}$ for $\mathbf{x} \in \mathfrak{X}$. Let $\mathbf{z}(\boldsymbol{p}, \boldsymbol{q}) \in C^{2,2}$ for $\boldsymbol{p} \in \mathfrak{P}, \boldsymbol{q} \in \mathfrak{D}$. If the problem $\left(\alpha^{\prime}\right)$ has a solution $\varphi\left(t, c^{*}\right)$ on the interval $\left\langle a, b^{*}\right\rangle$, then let us determine (if possible) functions $\boldsymbol{c}_{\overline{\mathscr{V}}}=\boldsymbol{\sigma}\left(\boldsymbol{c}_{\mathscr{V}}\right)$ and $b=b\left(\boldsymbol{c}_{\mathscr{V}}\right)(\mathscr{V} \subset \mathscr{N}, v(\mathscr{V})=k)$ defined and of class $C^{2}$ for $\boldsymbol{c}_{\mathscr{V}} \in \mathfrak{E}_{\mathscr{V}}$, $\mathfrak{S}_{\mathscr{r}}$ a domain in $E^{k}$, such that

(a) $\mathfrak{S}_{0}=E\left[\mathbf{c} \mid \mathbf{c}=\boldsymbol{c}_{\mathscr{V}}+\boldsymbol{\sigma}\left(\boldsymbol{c}_{\mathscr{V}}\right), \boldsymbol{c}_{\mathscr{V}} \in \mathfrak{S}_{\mathscr{V}}\right] \subset \mathfrak{C}$,

(b) $\mathbf{u}\left(\boldsymbol{\varphi}(a, \boldsymbol{c}), \boldsymbol{\varphi}\left(b\left(\boldsymbol{c}_{\mathscr{V}}\right), \boldsymbol{c}\right)\right) \equiv 0$ for $\boldsymbol{c} \in \mathfrak{S}_{0}$,

(c) $c^{*} \in \mathfrak{S}_{0}, b\left(c^{*}\right)=b^{*}$.

If we choose other parameters $\gamma=\left(\gamma_{1}, \gamma_{2}, \ldots, \gamma_{k}\right)$ from a domain $\mathfrak{S}$ in $E^{k}$ for expressing $c \in \mathfrak{S}_{0}$, let $\boldsymbol{c}=\boldsymbol{c}(\gamma) \in C^{1}$ and $\mathfrak{S}_{0}=E[\boldsymbol{c} \mid \boldsymbol{c}=\boldsymbol{c}(\gamma), \gamma \in \mathbb{G}]$ (then $\chi\left(\boldsymbol{c}_{\boldsymbol{\gamma}}(\gamma)\right)=k$ for $\gamma \in(\mathfrak{G}$, i. e. the parameters $\gamma$ are essential). The other conditions $(\mathscr{C})$ have to be modified in the obvious way.

Lemma 4.1. Let the conditions $\left(\mathscr{C}^{\prime}\right)$ be fulfilled.

(1) Let the problem $\left(\alpha^{\prime}\right)$ have a solution $\varphi\left(t, c^{*}\right)=\varphi^{*}(t)$. 
Then, denoting $\varphi^{*}(a)=\varphi_{a}^{*}, \varphi^{*}(b)=\varphi_{b}^{*}, \varphi^{*}(0)=\varphi_{0}^{*}, \mathbf{z}\left(\varphi_{a}^{*}, \varphi_{b}^{*}\right)=\mathbf{z}^{*}$ and similarly for other expressions, there holds the equality

$$
-\left[\frac{\mathbf{D z}}{\mathbf{D} \mathbf{x}_{a}} \frac{\mathrm{d} \varphi_{a}^{*}}{\mathrm{~d} t}+\frac{\mathbf{D z}}{\mathbf{D} \mathbf{x}_{b}} \frac{\mathrm{d} \varphi_{0}^{*}}{\mathrm{~d} t}\right]+\left[\frac{\mathbf{D z}}{\mathbf{D} \mathbf{x}_{a}} \frac{\mathbf{D} \varphi_{a}^{*}}{\mathbf{D c}}+\frac{\mathbf{D} z^{*}}{\mathbf{D} \mathbf{x}_{b}} \frac{\mathbf{D} \varphi_{b}}{\mathbf{D} \mathbf{c}}\right] \frac{\mathrm{d} \varphi_{0}^{*}}{\mathrm{~d} t}=0 .
$$

(2) Let the problem $\left(\alpha^{\prime}\right)$ have a k-parametric solution $\tilde{\varphi}(t)=\varphi(t, c(\gamma))$ on the interval $\langle a, b(\gamma)\rangle$ for $\gamma \in \mathbb{G}, \mathbb{G}$ a domain in $E^{k}$.

Then there holds the equality (denoting $\tilde{\mathbf{z}}($ or $\tilde{\mathbf{z}}(\gamma))=\mathbf{z}\left(\tilde{\boldsymbol{\varphi}}_{a}, \tilde{\varphi}_{b}\right)$ and so on)

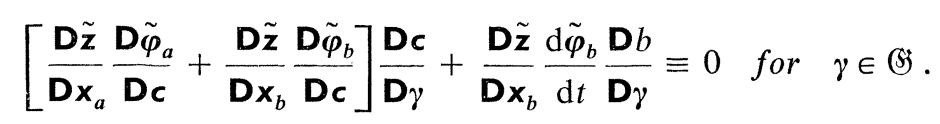

Proof of the assertion (1). If $\varphi\left(t, c^{*}\right)$ is a solution of problem $\left(\alpha^{\prime}\right)$, then the function $\varphi\left(t-\delta, \varphi\left(\delta, \mathrm{c}^{*}\right)\right)$ represents according to the group property of dynamical systems the same solution for all real numbers $\delta$ such that $\varphi\left(\delta, c^{*}\right) \in \mathfrak{C}$. Thus

$$
\mathbf{z}\left(\varphi\left(a-\delta, \varphi\left(\delta, c^{*}\right)\right), \varphi\left(b-\delta, \varphi\left(\delta, c^{*}\right)\right)\right) \equiv 0 .
$$

Differentiating (4.7) with respect to $\delta$ and then putting $\delta=0$ we get (4.5).

Proof of the assertion (2). By assumption

$$
\mathbf{z}(\varphi(a, c(\gamma)), \varphi(b(\gamma), c(\gamma))) \equiv 0 \text { for } \gamma \in \mathfrak{G} .
$$

Differentiating (4.8) with respect to $\gamma$, we get (4.6).

Lemma 4.2. Let the assumptions $\left(\mathscr{C}^{\prime}\right)$ be fulfilled. A solution $\varphi\left(t, c^{*}\right)$ of the equation (4.3') is constant if and only if

$$
\frac{\mathrm{d} \varphi}{\mathrm{d} t}\left(t_{0}, \mathrm{c}^{*}\right)=0 \text { for some } t_{0} \in \mathfrak{S}_{0} .
$$

The proof is evident.

Corollary 4.1. Let the assumption of the assertion (2) in Lemma 4.1 be fulfilled. Then there hold the inequalities

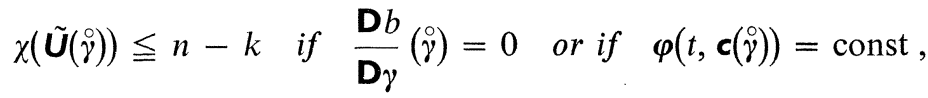

$$
\begin{aligned}
& \chi(\tilde{\mathbf{U}}(\stackrel{\circ}{\gamma})) \leqq n-k+1
\end{aligned}
$$

where

$$
\tilde{\mathbf{U}}(\gamma) \equiv \frac{\mathbf{D} \tilde{\mathbf{z}}}{\mathbf{D} \mathbf{x}_{a}} \frac{\mathbf{D} \tilde{\varphi}_{a}}{\mathbf{D} \mathbf{c}}+\frac{\mathbf{D} \tilde{\mathbf{z}}}{\mathbf{D} \mathbf{x}_{b}} \frac{\mathbf{D} \tilde{\varphi}_{b}}{\mathbf{D c}}
$$

and $\dot{\gamma} \in \mathfrak{G}$.

Proof. When $\frac{\mathbf{D} b}{\mathbf{D} \gamma}=0$ or $\varphi\left(t, c\left({ }^{\circ}\right)\right)=$ const, then by (1.6) the equation

$$
\tilde{\mathbf{U}}(\gamma) \xi=0
$$


has, according to (4.6) and $\chi\left(c_{\gamma}(\gamma)\right)=k$, at least $k$ linearly independent solutions whence by the known theorem of linear algebra the inequality (4.9) follows immediately.

$$
\begin{aligned}
\text { When } \frac{\mathbf{D} b}{\mathbf{D} \gamma} \neq 0 \text { and } \boldsymbol{\varphi}(t, \boldsymbol{c}(\stackrel{\circ}{\gamma})) \neq \text { const, then evidently } \\
\qquad \chi\left(\frac{\mathrm{d} \boldsymbol{\varphi}}{\mathrm{d} t}(b(\stackrel{\circ}{\gamma}), \boldsymbol{c}(\stackrel{\circ}{\gamma})) \frac{\mathbf{D} b}{\mathbf{D} \gamma}(\stackrel{\circ}{\gamma})\right)=1 .
\end{aligned}
$$

Then there clearly exists a $k \times(k-1)$ matrix $\boldsymbol{K}(\gamma)$ of rank $k-1$ such that

Since

$$
\frac{\mathbf{D} \tilde{\mathbf{z}}}{\mathbf{D} \mathbf{x}_{b}}(\stackrel{\circ}{\gamma}) \frac{\mathrm{d} \boldsymbol{\varphi}}{\mathrm{d} t}(b(\stackrel{\gamma}{\gamma}) \cdot \boldsymbol{c}(\stackrel{\circ}{\gamma})) \frac{\mathbf{D} b}{\mathbf{D} \gamma}(\stackrel{\circ}{\gamma}) \boldsymbol{K}(\stackrel{\circ}{\gamma})=0 .
$$

$$
\tilde{\mathbf{U}}\left(\stackrel{\circ}{\gamma)} \frac{\mathbf{D c}}{\mathbf{D} \gamma}(\stackrel{\circ}{\gamma}) \boldsymbol{K}(\stackrel{\circ}{\gamma})=0\right.
$$

and

$$
\chi\left(\frac{\mathbf{D} \mathbf{c}}{\mathbf{D} \gamma}(\gamma) \boldsymbol{K}(\gamma)\right)=k-1,
$$

the inequality follows by the same argument as above.

Let us now introduce a very important notion. We shall say that a boundary value problem $\left(\alpha^{\prime}\right)$ has the property $(\mathscr{P})$ if there holds: If the problem $\left(\alpha^{\prime}\right)$ has a solution $\varphi\left(t, c^{*}\right)$ on a fixed interval $\left\langle a, b^{*}\right\rangle$ then $\varphi\left(t, c^{*}\right)$ is as well a solution of the problem

$$
\dot{\boldsymbol{x}}=\boldsymbol{h}(\mathbf{x}), \mathbf{z}\left(\mathbf{x}(a+\delta), \mathbf{x}\left(b^{*}+\delta\right)\right)=0
$$

for every $\delta$ such that $\varphi\left(a+\delta, c^{*}\right) \in \mathfrak{C}$.

It is obvious that we may interpret this circumstance in two different ways:

(1) $\varphi(t, c)$, being a solution of the problem $\left(\alpha^{\prime}\right)$ on a fixed interval $\left\langle a, b^{*}\right\rangle$, is also a solution on every displaced interval $\left\langle a+\delta, b^{*}+\delta\right\rangle$ (on which the function $\varphi(t, c)$ is defined);

(2) $\varphi(t, c)$ being a solution of the problem $\left(\alpha^{\prime}\right)$ on a fixed interval $\left\langle a, b^{*}\right\rangle, \varphi\left(t+\delta, c^{*}\right)$ is a solution of the same problem on the same interval (for all $\delta$, for which $\varphi\left(t+\delta, c^{*}\right)$ is defined).

Lemma 4.3. Let the assumptions $\left(\mathscr{C}^{\prime}\right)$ be fulfilled. $\varphi\left(t, c^{*}\right)$ being any solution of the problem $\left(\alpha^{\prime}\right)$ (on a fixed interval $\left.\left\langle a, b^{*}\right\rangle\right)$, the problem $\left(\alpha^{\prime}\right)$ has the property $(\mathscr{P})$ if and only if

$$
\frac{\mathbf{D z}}{\mathbf{D} \mathbf{x}_{a}}\left(\varphi_{a+\delta}^{*}, \varphi_{b+\delta}^{*}\right) \frac{\mathrm{d} \varphi_{a+\delta}}{\mathrm{d} t}+\frac{\mathbf{D z}}{\mathbf{D} \mathbf{x}_{b}}\left(\varphi_{a+\delta}^{*}, \varphi_{b+\delta}^{*}\right) \frac{\mathrm{d} \varphi_{b+\delta}}{\mathrm{d} t} \equiv 0
$$

for every $\delta$ such that $\varphi\left(a+\delta, \mathrm{c}^{*}\right) \in(\sqrt{ }$.

The proof is easy. 
Corollary 4.2. If $\varphi^{*}(t)$ is a solution of a problem $\left(\alpha^{\prime}\right)$ which has the property $(\mathscr{P})$, then there holds the equality

$$
\frac{\mathbf{D z}}{\mathbf{D} \mathbf{x}_{a}} \frac{\mathrm{d} \varphi_{a}^{*}}{\mathrm{~d} t}+\frac{\mathbf{D} z^{*}}{\mathbf{D} \mathbf{x}_{b}} \frac{\mathrm{d} \varphi_{b}^{*}}{\mathrm{~d} t}=0 .
$$

Lemma 4.4. Let the assumptions $\left(\mathscr{C}^{\prime}\right)$ be fulfilled. Let a problem $\left(\alpha^{\prime}\right)$ have the property $(\mathscr{P})$. Let this problem have a non-constant solution $\varphi\left(t, c^{*}\right)=\varphi^{*}(t)$ on an interval $\left\langle a, b^{*}\right\rangle$.

Then the given problem $\left(\alpha^{\prime}\right)$ has on the interval $\left\langle a, b^{*}\right\rangle$ (at least) a one-parametric family of solutions $\varphi\left(t, \varphi\left(a+\delta, c^{*}\right)\right)$ for all $\delta$ such that $\varphi\left(a+\delta, c^{*}\right) \in \mathfrak{E}$.

Proof follows readily from the second interpretation of the property $(\mathscr{P})$ and from the group property of solutions of a dynamical system.

Corollary 4.3. Let the assumptions of Lemma 4.4 be fulfilled. Then the jacobian of the system

$$
\mathbf{z}\left(\varphi(a, \mathbf{c}), \varphi\left(b^{*}, \mathbf{c}\right)\right)=0
$$

with respect to $\mathrm{c}$ is vanishing at the point $\mathrm{c}=\mathrm{c}^{*}$.

This is an immediate consequence of the fact that the system (4.13) has (at least a one-parametric family of solutions $c=\varphi\left(a+\delta, c^{*}\right)$. (One may show it also by means of (4.5), (4.12) and Lemma (4.2.)

Corollary 4.4. For proving the existence of a solution of a problem $(\alpha)$ with the property $(\mathscr{P})$ for all $\varepsilon \in \mathbb{E}$ Theorems 3.1 or 3.2 cannot be used.

This is clear from the fact that by these theorems the existence of an isolated solution is proved, which contradicts Lemma 4.4 .

(On the other hand when the problem $(\alpha)$ has not the property $(\mathscr{P})$ we have no reason to expect non-validity of Theorems 3.1 or 3.2.)

Let a problem $(\alpha)$ with the property $(\mathscr{P})$ be given. Let us suppose that this problem has a non-constant solution $\varphi\left(t, c^{*}(\varepsilon), \varepsilon\right)$ on the interval $\left\langle a, b^{*}(\varepsilon)\right\rangle$ for $\varepsilon \in \mathcal{E}$. Then $\varphi_{0}^{*}(t)=\varphi\left(t, c^{*}(0), 0\right)$ is a solution of the shortened problem $\left(\alpha_{0}\right)$. Let us suppose that this solution also is non-constant and say that in particular the component $\varphi_{0, l}^{*}(t)$ is non-constant. Then there exists obviously $t_{0} \in \mathfrak{Z}$ such that $\dot{\varphi}_{0, l}\left(t_{0}\right) \neq 0$. Denote $\lambda=\varphi_{0, l}^{*}\left(t_{0}\right)$ and let $\boldsymbol{d}_{0}=\varphi_{0}^{*}\left(t_{0}\right) \in \mathfrak{C}, \varphi_{0}^{*}\left(t_{0}+t\right) \subset \mathfrak{X}$ for $t \in \mathfrak{Z}$. By the property $(\mathscr{P})$, $\varphi_{0}^{*}\left(t, \boldsymbol{d}_{0}\right)$ is also a solution of the problem $\left(\alpha_{0}\right)$. According to the continuous dependence of a solution on a parameter, there exists for sufficiently small $\varepsilon$ also a solution $\varphi\left(t, \boldsymbol{d}^{*}(\varepsilon), \varepsilon\right)$ of the problem $(\alpha)$ such that $\varphi_{l}\left(a, \boldsymbol{d}^{*}(\varepsilon), \varepsilon\right)=\lambda$. Thus, by fixing from the beginning $c_{l}=\lambda$, we fix only one solution from (at least) a one-parametric family of solutions of the problem $(\alpha)$. Instead of the component $c_{l}$ of the initial vector we must then choose as a new variable the variation $\tau^{*}(\varepsilon)=b^{*}(\varepsilon)-b^{*}(0)$ of the interval $\left\langle a, b^{*}(0)\right\rangle$. 
Let us now clarify the position of boundary valye problems with periodic boundary conditions among other boundary value problems with the property $(\mathscr{P})$.

Lemma 4.5. Let $\mathbf{z}\left(\mathbf{x}_{a}, \mathbf{x}_{b}\right)$ be given. Let a boundary value problem $\left(\alpha^{\prime}\right)$ have the property $(\mathscr{P})$ for an arbitrary function $\boldsymbol{h}(\mathbf{x})$ for which the boundary value problem $\left(\alpha^{\prime}\right)$ fulfils the conditions $\left(\mathscr{C}^{\prime}\right)$ with the same sets $\mathfrak{E}, \mathfrak{X}, \mathfrak{E}, \mathfrak{P}$ and $\mathfrak{S}_{\text {. Then }}$

$$
\mathbf{z}\left(\mathbf{x}_{a}, \mathbf{x}_{b}\right)=0 \Rightarrow \mathbf{x}_{a}-\mathbf{x}_{b}=0 \text {. }
$$

Proof. Let us suppose that there exist two points $\boldsymbol{x}_{1}, \boldsymbol{x}_{2}$ (not necessarily differing from each other) such that $\mathbf{x}_{1} \in \mathfrak{P}, \mathbf{x}_{2} \in \mathfrak{S}_{2}$ and $\mathbf{z}\left(\mathbf{x}_{1}, \mathbf{x}_{2}\right)=0$. Now, let us suppose that $\boldsymbol{x}_{1} \neq \mathbf{x}_{2}$. Choose constant vectors $\boldsymbol{h}_{1}, \boldsymbol{h}_{2} \in E^{n}$ such that

$$
\frac{\mathrm{Dz}}{\mathrm{D} \mathbf{x}_{a}}\left(\mathrm{x}_{1}, \mathrm{x}_{2}\right) \boldsymbol{h}_{1}+\frac{\mathrm{Dz}}{\mathrm{D} \mathbf{x}_{b}}\left(\mathrm{x}_{1}, \mathbf{x}_{2}\right) \boldsymbol{h}_{2} \neq 0 .
$$

Join the points $\mathbf{x}_{1}, \mathbf{x}_{2}$ by a simple $\operatorname{arc} \mathbf{x}=\xi(t)$ of class $C^{2}$ for $t \in \mathfrak{I}$ so that $\xi(a)=\mathbf{x}_{1}$, $\xi(b)=\boldsymbol{x}_{2}, \frac{\mathrm{d} \xi}{\mathrm{d} t}(a)=\boldsymbol{h}_{1}, \frac{\mathrm{d} \xi}{\mathrm{d} t}(b)=\boldsymbol{h}_{2}$ and $\xi(t) \in \mathfrak{X}$ for $t \in \mathfrak{Z}$. Vectors $\boldsymbol{h}_{1}, \boldsymbol{h}_{2}$ and the $\operatorname{arc} \xi(t)$ with prescribed properties evidently exist.

Denote $X=E[\mathbf{x} \mid \mathbf{x}=\boldsymbol{\xi}(t), t \in \mathfrak{I}]$. To each point $\mathbf{x}_{0}=\boldsymbol{\xi}\left(t_{0}\right) \in X$ co-ordinate the vector $\frac{\mathrm{d} \xi}{\mathrm{d} t}\left(t_{0}\right)=\boldsymbol{h}\left(\mathbf{x}_{0}\right)$. The function $\boldsymbol{h}(\mathbf{x})$ being defined and of class $C^{2}$ on the compact set $X$, may be continued onto the whole set $\mathfrak{X}$. Thus a function $\boldsymbol{h}(\mathbf{x})$ is defined on $\mathfrak{X}$ such that the boundary value problem $\left(\alpha^{\prime}\right)$, defined by functions $\boldsymbol{h}$ and $\mathbf{z}$, fulfils the conditions $\left(\mathscr{C}^{\prime}\right)$ and has a solution $\varphi^{*}(t)=\xi(t)$ such that

$$
\boldsymbol{\varphi}^{*}(a)=\mathbf{x}_{1}, \quad \boldsymbol{\varphi}^{*}(b)=\mathbf{x}_{2}, \quad \dot{\varphi} *(a)=\boldsymbol{h}\left(\mathbf{x}_{1}\right)=\boldsymbol{h}_{1}, \quad \dot{\varphi} *(b)=\boldsymbol{h}\left(\mathbf{x}_{2}\right)=\boldsymbol{h}_{2} .
$$

But by Corollary 4.2 in virtue of (4.14) the defined boundary value problem cannot have the property $(\mathscr{P})$. Thus, it has to be $\boldsymbol{x}_{1}=\boldsymbol{x}_{2}$.

It would be very interesting to know under what conditions a boundary value problem $(\alpha)$ has the property $(\mathscr{P})$ for all $\varepsilon \in \mathcal{E}$. It can be shown that this also happens for other than periodic boundary conditions, e. g. it may easily be verified that the boundary value problem

$$
\dot{\mathbf{x}}=\mathbf{A x}+\varepsilon \boldsymbol{B} \mathbf{x}, \quad \mathbf{M} \mathbf{x}(a)+\mathbf{N} \mathbf{x}(b)=0
$$

where $\chi(\boldsymbol{M}: \mathbf{N})=n$ and matrices $\boldsymbol{A}$ and $\boldsymbol{B}$ commute with matrices $\boldsymbol{M}$ and $\mathbf{N}$, has the property $(\mathscr{P})$ for all $\varepsilon$.

We shall see that in the main theorems our method fails when the solution of the shortened boundary value problem is constant. It is natural to seek then a solution of the same character (i. e. a constant one) of the perturbed system. This problem is solved by the following

Lemma 4.6. Let the conditions $(\mathscr{C})$ be fulfilled. The boundary value problem $(\alpha)$ 
has a constant solution $\varphi\left(t, c^{*}(\varepsilon), \varepsilon\right)=c^{*}(\varepsilon)$ if and only if the system of $2 n$ equations

$$
f(c)+\varepsilon \mathbf{g}(\boldsymbol{c}, \varepsilon)=0, \quad \mathbf{w}(\boldsymbol{c}, \boldsymbol{c}, \varepsilon) \equiv \mathbf{u}(\mathbf{c}, \boldsymbol{c})+\varepsilon \mathbf{v}(\boldsymbol{c}, \boldsymbol{c}, \varepsilon)=0
$$

has a real solution $c=c^{*}(\varepsilon)$.

The proof is evident.

In the sequel we have to distinguish the case when the shortened boundary value problem $\left(\alpha_{0}\right)$ associated to the given boundary value problem $(\alpha)$ has a $k$-parametric family of solutions $\varphi(t, c(\gamma)), \gamma \in \mathfrak{G}$ on the interval $\langle a, b\rangle$ of constant length and the case when such a family exists on the interval $\langle a, b(\gamma)\rangle$ whose length depends on the parameters $\gamma$. The reason is the following: In Theorems 4.1 and 4.3 the number of linearly independent solutions of the variational boundary value problem $(\mu)$

$$
\begin{gathered}
\dot{\mathbf{z}}=\boldsymbol{f}_{\boldsymbol{x}}(\tilde{\varphi}(t)) \mathbf{z}, \quad \tilde{\varphi}(t)=\boldsymbol{\varphi}(t, \boldsymbol{c}(\gamma)), \\
\frac{\mathrm{D} \tilde{u}}{\mathbf{D x}_{\boldsymbol{a}}} \mathbf{z}(a)+\frac{\mathbf{D} \tilde{\mathbf{u}}}{\mathbf{D} \boldsymbol{x}_{b}} \mathbf{z}(b)=0, \quad \tilde{\mathbf{u}}=\mathbf{u}\left(\tilde{\varphi}_{a}, \tilde{\varphi}_{b}\right),
\end{gathered}
$$

is of great importance. By Theorem 1.4 this number equals $n-\chi(\tilde{\mathbf{U}})$, where

$$
\tilde{\mathbf{U}}(\gamma) \equiv \frac{\mathrm{Du}}{\mathrm{D} \mathbf{x}_{a}} \frac{\mathbf{D} \tilde{\varphi}_{a}}{\mathrm{Dc}}+\frac{\mathbf{D u}}{\mathrm{Dx}} \frac{\mathbf{D} \tilde{\varphi}_{b}}{\mathrm{Dc}} .
$$

By Corollary 4.1 in the first case $(b=$ const $) \chi(\tilde{\mathbf{U}}(\gamma)) \leqq n-k$ whereas in the second case $(b(\gamma) \neq$ const $) \chi(\tilde{\boldsymbol{U}}(\gamma)) \leqq n-k+1$ for $\gamma \in \mathbb{G}$. We shall suppose that the ranks of the matrices in question attain their maximal values. Hence in the first case the problem $(\mu)$ has exactly $k$ linearly independent solutions, whereas in the second case it has exactly $k-1$ linearly independent solutions only.

Theorem 4.1. Let an interval $\mathfrak{I}=\left\langle a, b_{0}\right\rangle$ be given. Consider the boundary value problem $(\alpha)$

$$
\begin{gathered}
\dot{x}=f(\mathbf{x})+\varepsilon \mathbf{g}(\mathbf{x}, \varepsilon), \\
\mathbf{w}(\mathbf{x}(a), \mathbf{x}(b(\varepsilon)), \varepsilon) \equiv \mathbf{u}\left(\mathbf{x}_{a}, \mathbf{x}_{b}\right)+\varepsilon \mathbf{v}\left(\mathbf{x}_{a}, \mathbf{x}_{b}, \varepsilon\right)=0
\end{gathered}
$$

where the function $b(\varepsilon)$ with $b(0)=b_{0}$ has to be determined suitably. Let the following assumptions be fulfilled.

(I) Let the problem $(\alpha)$ have the property $(\mathscr{P})$ for all $\left.\varepsilon \in \mathcal{F}=\left\langle 0, \varepsilon_{0}\right\rangle, \varepsilon_{0}\right\rangle 0$.

(II) The same as (I) in Theorem 3.2.

(III) (a) Denote $\sqrt{5}$ an open set in $E^{n}$. Let solutions $\boldsymbol{\eta}(t, \mathrm{c})$ of

$$
\dot{y}=f(y)
$$

with $\boldsymbol{\eta}(a, c)=\mathbf{c}$ exist and remain in $\mathfrak{X}$ for $t \in \mathfrak{I}_{0}=(A, B) \supset\left\langle a, b_{0}\right\rangle$ and for all $c \in \mathfrak{C}$.

(b) Let equations

$$
\boldsymbol{u}(\boldsymbol{\eta}(a, c), \boldsymbol{\eta}(b, c))=0
$$


(where we consider $b$ as an unknown) have a real solution $b=b_{0}, c_{\bar{Y}}=\sigma_{0}\left(c_{r}\right)($ that means that the number $k$ cannot be increased by equating $b$ to some nonconstant function of $\left.\boldsymbol{c}_{\mathscr{V}}\right), \mathscr{V} \subset \mathscr{N}, v(\mathscr{V})=k, 2 \leqq k \leqq n$.

(c) Denote $c_{\gamma}=\gamma$ and $\varrho_{0}(\gamma)=\gamma+\sigma_{0}(\gamma)$. Let $\sigma_{0}(\gamma) \in C^{2}$ for $\gamma \in(\mathcal{G}$, (F) an' open set in $E^{k}$ such that $\mathfrak{D}=E\left[\mathbf{c} \mid \mathbf{c}=\varrho_{0}(\gamma), \gamma \in \mathfrak{G}\right] \subset \mathfrak{C}$. Denote $\tilde{\boldsymbol{\eta}}(t)=\boldsymbol{\eta}\left(t, \varrho_{0}(\gamma)\right)$ so that $\tilde{\eta}(t)$ is a k-parametric solution of the boundary value problem $\left(\alpha_{0}\right)$ given by (4.3) and (4.4) on the interval $\left\langle a, b_{0}\right\rangle$.

(d) Let $\tilde{\boldsymbol{\eta}}(a) \in \mathfrak{P}, \boldsymbol{\eta}(b) \in \mathfrak{Q}$. for $\boldsymbol{\gamma} \in \mathfrak{G}$.

(e) Let $l \in \mathscr{V}$. Put $c_{l}=\lambda \in E\left[c_{l} \mid c_{l}=\tilde{\eta}_{l}(a), \gamma \in \mathbb{B}\right]$.

Denote $\alpha=\gamma-\lambda, \hat{\sigma}_{0}(\alpha)=\sigma_{0}(\alpha+\lambda), \hat{\varrho}_{0}(\alpha)=\varrho_{0}(\alpha+\lambda), \hat{\eta}(t)=\boldsymbol{\eta}\left(t, \hat{\varrho}_{0}(\alpha)\right)$. (Thus, $\hat{\boldsymbol{\eta}}(t)$ is a $(k-1)$-parametric solution of the problem $\left(\alpha_{0}\right)$ with $c_{l}=\lambda$.)

(IV) Let the variational boundary value problem $(\mu)$

$$
\dot{\mathbf{z}}=\boldsymbol{f}_{\boldsymbol{x}}(\tilde{\boldsymbol{\eta}}(t)) \mathbf{z},
$$

$$
\frac{\mathbf{D} \tilde{u}}{\mathbf{D} \boldsymbol{x}_{\boldsymbol{a}}} \mathbf{z}(a)+\frac{\mathbf{D} \tilde{\mathbf{u}}}{\mathbf{D} \mathbf{x}_{\boldsymbol{b}}} \mathbf{z}(b)=0
$$

where

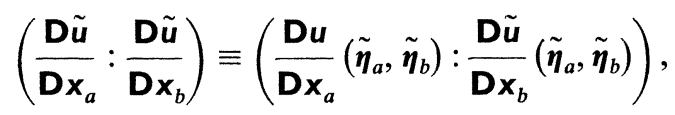

have exactly $k$ linearly independent solutions for $\gamma \in \mathbb{B}$.

(V) We shall show that there exist a set $\mathfrak{H}_{0}, \mathfrak{A}_{0}$ a domain in $E^{k-1}$ such that $E\left[\gamma \mid \gamma=\alpha+\lambda, \alpha \in \mathfrak{H}_{0}\right] \subset \mathfrak{G}$, a set $\mathscr{S} \subset \mathcal{N}, v(\mathscr{S})=k$ and $a k \times(n-k)$ matrix $T(\alpha)$ such that

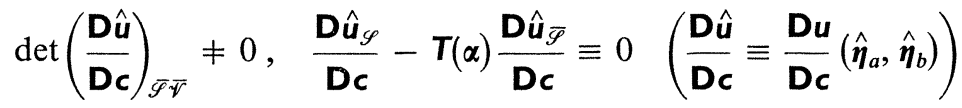

for $\alpha \in \mathfrak{A}_{0}$.

Further we shall show that there exists a set $\mathfrak{A}_{1}, \mathfrak{H}_{1}$ a domain in $\mathfrak{H}_{0}$ and a $k \times n$ matrix $\Psi(t, \alpha)$ whose rows are formed by $k$ solutions of the boundary value problem $\left(\hat{\mu}^{*}\right)$ (adjoint to boundary value problem $(\mu)$ in which $c_{l}=\lambda$ is put)

$$
\begin{gathered}
\dot{w}^{\prime}=-\boldsymbol{w}^{\prime} \boldsymbol{f}_{\mathbf{x}}(\hat{\boldsymbol{\eta}}(t)), \\
\mathbf{w}^{\prime}(a) \boldsymbol{P}^{\prime}(\boldsymbol{\alpha})+\mathbf{w}^{\prime}\left(b_{0}\right) \mathbf{Q}^{\prime}(\boldsymbol{\alpha})=0
\end{gathered}
$$

where $n \times n$ matrices $\mathbf{P}(\alpha), \mathbf{Q}(\alpha)$ satisfy conditions

$$
-\frac{\mathbf{D} \hat{u}}{\mathbf{D} x_{a}} \mathbf{P}^{\prime}(\boldsymbol{\alpha})+\frac{\mathbf{D} \hat{u}}{\mathbf{D x}_{b}} \mathbf{Q}^{\prime}(\boldsymbol{\alpha}) \equiv 0, \quad \chi(\boldsymbol{P}(\boldsymbol{\alpha}): \mathbf{Q}(\boldsymbol{\alpha}))=n
$$

for $\alpha \in \mathfrak{A}_{1}$ and which fulfils the condition

$$
\Psi\left(b_{0}, \alpha\right) \equiv \frac{\mathbf{D} \hat{\mathbf{u}}_{\mathscr{S}}}{\mathbf{D} \mathbf{x}_{\boldsymbol{b}}}-\boldsymbol{T}(\alpha) \frac{\mathbf{D} \hat{\mathbf{u}}_{\overline{\mathscr{Y}}}}{\mathbf{D} \mathbf{x}_{\boldsymbol{b}}} .
$$


(a) Let the system

$$
\begin{gathered}
v_{0} \boldsymbol{\Psi}\left(b_{0}, \boldsymbol{\alpha}_{0}\right) \dot{\boldsymbol{\eta}}\left(b_{0}, \hat{\varrho}_{0}\left(\alpha_{0}\right)\right)+ \\
+\int_{a}^{b_{0}} \boldsymbol{\Psi}\left(s, \alpha_{0}\right) \boldsymbol{g}\left(\boldsymbol{\eta}\left(s, \hat{\varrho}_{0}\left(\alpha_{0}\right)\right), 0\right) \mathrm{d} s+\mathbf{v}_{0}-\boldsymbol{T}\left(\boldsymbol{\alpha}_{0}\right) \mathbf{v}_{0 \overline{\mathscr{S}}}=0
\end{gathered}
$$

where $\mathbf{v}_{0}=\mathbf{v}\left(\boldsymbol{\eta}\left(a, \hat{\varrho}_{0}\left(\boldsymbol{\alpha}_{0}\right)\right), \boldsymbol{\eta}\left(b_{0}, \hat{\varrho}_{0}\left(\boldsymbol{\alpha}_{0}\right)\right), 0\right)$ have a real solution $v_{0}=v_{0}^{*}, \boldsymbol{\alpha}_{0}=\boldsymbol{\alpha}_{0}^{*}$.

(b) Let $\alpha_{0}^{*} \in \mathfrak{A}_{1}$.

(c) Let $\dot{\eta}_{l}\left(a, \hat{\varrho}_{0}\left(\alpha_{0}^{*}\right)\right) \neq 0$.

(d) Let the jacobian of the system (4.26) with respect to $v_{0}, \alpha_{0}$ be nonvanishing at the point $v_{0}=v_{0}^{*}, \alpha_{0}=\alpha_{0}^{*}$.

(VI) Denote $\mathfrak{X}=E\left[\mathbf{x} \mid \| \mathbf{x}-\boldsymbol{\eta}\left(t, \hat{\varrho}_{0}\left(\boldsymbol{\alpha}_{0}^{*}\right) \|<\varrho, t \in\left\langle a, b_{0}+\delta\right\rangle\right], \varrho>0, \delta>0\right.$. Let $\boldsymbol{f}(\mathbf{x}) \in C^{2}, \boldsymbol{g}(\mathbf{x}, \varepsilon) \in C^{1,1}$ for $\mathbf{x} \in \mathfrak{X}, \varepsilon \in \mathfrak{E}$.

Then there exists for sufficiently small $\varepsilon>0$ a unique function $b^{*}(\varepsilon)=b_{0}+$ $+\varepsilon v^{*}(\varepsilon), v^{*}(\varepsilon) \in C^{1}$ such that there exists a unique solution $\xi\left(t, c^{*}(\varepsilon), \varepsilon\right)$ of the problem $(\alpha)$ with $\xi\left(a, c^{*}(\varepsilon), \varepsilon\right)=\mathbf{c}^{*}(\varepsilon) \in C^{1}, c_{l}^{*}(\varepsilon)=\lambda$ on the interval $\left\langle a, b^{*}(\varepsilon)\right\rangle$ which tends to the solution $\boldsymbol{\eta}\left(t, \hat{\varrho}_{0}\left(\alpha_{0}^{*}\right)\right)$ of the problem $\left(\alpha_{0}\right)$ as $\varepsilon \rightarrow 0$.

Proof. We shall explain in more detail those parts of the proof only in which the proof of this theorem differs from the proof of Theorem 3.2.

Clearly $\boldsymbol{\eta}_{c}\left(t, \varrho_{0}(\gamma)\right)$ is a fundamental matrix of solutions of (4.19). By (IV), (IIb), (IIIc) and Theorem 1.4

$$
\chi\left(\frac{\mathbf{D} \tilde{u}}{\mathbf{D} \boldsymbol{c}}\right) \equiv \chi\left(\frac{\mathbf{D} \tilde{u}}{\mathbf{D x}} \frac{\mathbf{D} \tilde{\boldsymbol{\eta}}_{a}}{\mathbf{D} \boldsymbol{c}}+\frac{\mathbf{D} \tilde{u}}{\mathbf{D} \boldsymbol{x}_{b}} \frac{\mathbf{D} \tilde{\boldsymbol{\eta}}_{b}}{\mathbf{D c}}\right)=n-k .
$$

In particular there exist sets $\mathscr{S} \subset \mathcal{N}, \mathscr{V}_{1} \subset \mathscr{N}, v(\mathscr{S})=v\left(\mathscr{V}_{1}\right)=k$, such that

$$
\operatorname{det}\left(\frac{\mathbf{D} \tilde{u}}{\mathbf{D c}}\right)_{\overline{\mathcal{P}}_{\bar{V}_{1}}} \neq 0 \text {. }
$$

Analogously as in Theorem 3.2 it may be shown that $\mathscr{V}_{1}=\mathscr{V}$ may be supposed without loss of generality.

Obviously putting $c_{l}=\lambda \in E\left[c_{l} \mid c_{l}=\eta_{l}\left(a, \varrho_{0}(\gamma)\right), \gamma \in(\$)\right]$ in (4.27) and (4.28) it holds for $\alpha, \alpha+\lambda \in \mathbb{G}$,

$$
\begin{aligned}
& \chi\left(\frac{\mathbf{D} \hat{u}}{\mathbf{D} \boldsymbol{c}}\right)=n-k, \\
& \operatorname{det}\left(\frac{\mathbf{D} \hat{u}}{\mathbf{D} \mathbf{c}}\right)_{\overline{\mathscr{J}} \overline{\mathfrak{V}}} \neq 0 .
\end{aligned}
$$

Thus there exists a $k \times(n-k)$ matrix $\boldsymbol{T}(\alpha)$ such that

$$
\frac{\mathrm{D} \hat{\mathrm{u}}_{\mathscr{S}}}{\mathrm{Dc}} \equiv T(\alpha) \frac{\mathrm{D} \hat{\mathrm{u}}_{\overline{\mathscr{S}}}}{\mathrm{Dc}}
$$


whence it follows in particular

$$
\begin{aligned}
& \left(\frac{\mathbf{D} \hat{u}}{\mathbf{D c}}\right)_{\mathscr{S} \overline{\mathscr{V}}}-\mathbf{T}(\alpha)\left(\frac{\mathbf{D} \hat{u}}{\mathbf{D c}}\right)_{\overline{\mathscr{S}} \overline{\mathscr{V}}} \equiv 0,
\end{aligned}
$$

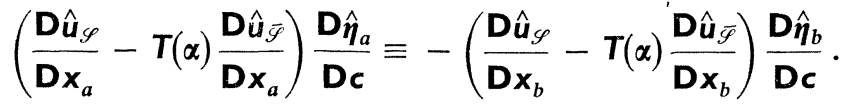

Putting $c_{l}=\lambda$ into (4.19) and (4.20) we get the boundary value problem $(\hat{\mu})$ which again has clearly exactly $k$ linearly independent solutions for $\alpha \in \mathfrak{A}_{0}$. As in Theorem 3.2 the existence of the matrix $\Psi(t, \alpha)$ defined in (IV) for $\alpha \in \mathfrak{A}_{1}, \mathfrak{A}_{1}$ some domain in $\mathfrak{A}_{0}$, may be proved and namely that for $\alpha \in \mathfrak{H}_{1}$

$$
\boldsymbol{\Psi}(t, \alpha) \equiv\left(\frac{\mathbf{D} \hat{\mathbf{u}}_{\mathscr{S}}}{\mathbf{D} \boldsymbol{x}_{b}}-\bar{T}(\alpha) \frac{\mathbf{D} \hat{u}_{\bar{S}}}{\mathbf{D} \boldsymbol{x}_{b}}\right) \frac{\mathbf{D} \hat{\eta}_{b}}{\mathbf{D} \boldsymbol{c}}\left(\frac{\mathbf{D} \hat{\boldsymbol{\eta}}}{\mathbf{D c}}(t)\right)^{-1} .
$$

Now the equations (4.26) are completely defined for $\alpha \in \mathfrak{A}_{1}$ and we may find their solution $v_{0}=v_{0}^{*}, \alpha_{0}=\alpha_{0}^{*}$ which fulfils the assumptions $(\mathrm{Vb}, \mathrm{c}, \mathrm{d})$.

If there exists a solution $\xi\left(t, c^{(0)}(\varepsilon), \varepsilon\right)$ of the problem $(\alpha)$ which tends to the solution $\boldsymbol{\eta}^{*}(t)=\boldsymbol{\eta}\left(t, \varrho_{0}\left(\alpha_{0}^{*}+\lambda\right)\right)$ of the problem $\left(\alpha_{0}\right)$ fulfilling the condition $\left.(\mathrm{Vc})\right)$ as $\varepsilon \rightarrow 0$, then we know by previous considerations that there definitely exists in virtue of (I) and of the continuity a solution $\xi\left(t, c^{*}(\varepsilon), \varepsilon\right)$ of $(\alpha)$ which tends also to $\eta^{*}(t)$ and which satisfies the condition $c_{l}^{*}(\varepsilon)=\lambda$. Hence let us require

$$
\xi_{l}\left(a, c^{*}(\varepsilon), \varepsilon\right)=c_{l}^{*}(\varepsilon)=\lambda .
$$

Remark. In practice, it may sometimes be difficult to find at the begining a numerical value $c_{l}=\lambda$ such that the assumptions ( $\mathrm{Va}, \mathrm{b}, \mathrm{c}, \mathrm{d}$ ) would be fulfilled (if it is possible at all). Thus it may be more appropriate to keep $\lambda$ as a parameter and not to choose its value until the equations (4.26) are determined and then to try to choose its value in such a way that the assumptions ( $\mathrm{Va}, \mathrm{b}, \mathrm{c}, \mathrm{d}$ ) would be satisfied.

Now by (VI), Theorem 2.1 and the theorem of continuous dependence on initial values and parameters there exist a neighborhood $\mathfrak{D}_{1}\left(\mathbf{c}_{0}^{*}, \delta_{1}\right), \mathbf{c}_{0}^{*}=\hat{\varrho}_{0}\left(\alpha_{0}^{*}\right)$ and intervals

$$
\mathfrak{F}_{1}=\left\langle 0, \varepsilon_{1}\right\rangle, \quad 0<\varepsilon_{1} \leqq \varepsilon_{0}, \quad \mathfrak{B}_{1}=\left\langle b_{0}-\pi_{1}, b_{0}+\pi_{1}\right\rangle, \pi_{1}>0
$$

such that (1) every solution $\xi(t, c, \varepsilon)$ of (4.1) where $\varepsilon \in \mathfrak{F}_{1}$, with $\xi(a, c, \varepsilon)=c \in$ $\in \mathfrak{D}_{1}\left(c_{0}^{*}, \delta_{1}\right)$ remains in $\mathfrak{X}$ for $t \in \mathfrak{Z}_{0}$ and it may be written in the form

$$
\xi(a, c, \varepsilon)=\boldsymbol{\eta}(t, \boldsymbol{C}(t, \boldsymbol{c}, \varepsilon)),
$$

where $\boldsymbol{C}(t, \boldsymbol{c}, \varepsilon)$ is a solution of

$$
\Gamma(t)=c+\varepsilon \int_{a}^{t} \boldsymbol{\eta}_{c}^{-1}(s, \Gamma(s)) \boldsymbol{g}(\boldsymbol{\eta}(s, \Gamma(s)), \varepsilon) \mathrm{d} s,
$$


and (2) according to $\left(4.28^{\prime}\right)$

$$
\operatorname{det}\left(\frac{\mathbf{D} w}{\mathbf{D c}}\left(\xi_{a}, \xi_{b}, \varepsilon\right)\right)_{\overline{\mathscr{S}} \overline{\mathscr{V}}} \neq 0
$$

for $c \in \mathfrak{D}_{1}\left(\mathbf{c}_{0}^{*}, \delta_{1}\right), b \in \mathfrak{B}_{1}$ and $\varepsilon \in \mathfrak{F}_{1}$.

A solution $\xi(t, c, \varepsilon)$ of $(4.1)$ is a solution of the boundary value problem $(\alpha)$ if and only if

$$
w\left(\xi_{a}, \xi_{b}, \varepsilon\right)=0 .
$$

Inserting $c_{l}=\lambda$ into (4.35) we get (denoting $\mathscr{L}$ the complement of the index $l$ with respect to $\mathscr{V}$ )

$$
\mathbf{w}\left(\xi\left(a, \boldsymbol{c}_{\overline{\mathscr{V}}}+\boldsymbol{c}_{\mathscr{L}}+\lambda, \varepsilon\right), \quad \xi\left(b, \boldsymbol{c}_{\overline{\mathcal{V}}}+\boldsymbol{c}_{\mathscr{L}}+\lambda, \varepsilon\right), \varepsilon\right)=0 .
$$

Leaving equations from $\left(4.35^{\prime}\right)$ with indices from $\overline{\mathscr{S}}$ unchanged we have

$$
\mathbf{w}_{\overline{\mathscr{S}}}\left(\boldsymbol{\xi}\left(a, \boldsymbol{c}_{\overline{\mathscr{V}}}+\mathbf{c}_{\mathscr{L}}+\lambda, \varepsilon\right), \quad \xi\left(b, \boldsymbol{c}_{\overline{\mathscr{V}}}+\mathbf{c}_{\mathscr{L}}+\lambda, \varepsilon\right), \varepsilon\right)=0 .
$$

By (4.34) and the implicit function theorem there exist a neighborhood $\mathfrak{N}_{2}\left(\alpha_{0}^{*}, \delta_{2}\right)$, $0<\delta_{2} \leqq \delta_{1}$, and sets $\mathfrak{B}_{2}=\left\langle b_{0}-\pi_{2}, b_{0}+\pi_{2}\right\rangle, 0<\pi_{2} \leqq \pi_{1}, \quad \xi_{2}=\left\langle 0, \varepsilon_{2}\right\rangle$, $0<\varepsilon_{2} \leqq \varepsilon_{1}$ such that for $\boldsymbol{c}_{\mathscr{L}} \in \mathfrak{N}_{2}\left(\alpha_{0}^{*}, \delta_{2}\right), b \in \mathfrak{B}_{2}$ and $\varepsilon \in \mathfrak{F}_{2}$ there exists a solution

$$
\mathbf{c}_{\overline{\mathscr{V}}}=\boldsymbol{\sigma}\left(\mathbf{c}_{\mathscr{L}}, b, \varepsilon\right)
$$

of (4.36) such that (denoting again $\left.\boldsymbol{c}_{\mathscr{L}}=\alpha, \varrho(\alpha, b, \varepsilon)=\alpha+\lambda \dot{+} \boldsymbol{\sigma}(\boldsymbol{\alpha}, b, \varepsilon)\right), \boldsymbol{\sigma}(\boldsymbol{\alpha}, b, \varepsilon) \epsilon$ $\in C^{2,2,1}, \sigma\left(\alpha, b_{0}, 0\right)=\hat{\sigma}_{0}\left(\alpha_{0}\right)$ and $\varrho(\alpha, b, \varepsilon) \in \mathfrak{D}_{1}\left(c_{0}^{*}, \delta_{1}\right)$.

Inserting (4.37) into (4.36), denoting

$$
\begin{gathered}
\tau=b-b_{0}, \\
\mathfrak{g}(\alpha, b, \varepsilon)=\int_{a}^{b} \boldsymbol{\eta}_{c}^{-1}(s, \boldsymbol{C}(s, \varrho(\alpha, b, \varepsilon), \varepsilon)) \mathbf{g}(\boldsymbol{\eta}(s, \boldsymbol{C}(s, \varrho(\alpha, b, \varepsilon)), \varepsilon) \mathrm{d} s
\end{gathered}
$$

and making use of the mean value theorem we get the identity

$$
\boldsymbol{w}_{\overline{\mathscr{S}}}\left(\hat{\boldsymbol{\xi}}_{a}, \hat{\boldsymbol{\xi}}_{b}, \varepsilon\right) \equiv \mathbf{u}_{\overline{\mathscr{S}}}\left(\hat{\boldsymbol{\eta}}(a), \hat{\boldsymbol{\eta}}\left(b_{0}\right)\right)+\tau \int_{0}^{1}\left[\frac{\mathbf{D} \mathbf{u}_{\overline{\mathcal{S}}}}{\mathbf{D c}_{\overline{\mathcal{V}}}} \frac{\partial \boldsymbol{\sigma}}{\partial b}\right]_{\vartheta} \mathrm{d} \vartheta+
$$

$$
\begin{aligned}
+\varepsilon \int_{0}^{1}\left[\frac{\mathbf{D} \mathbf{u}_{\overline{\mathcal{S}}}}{\mathbf{D} \mathbf{c}_{\overline{\mathscr{V}}}} \frac{\partial \boldsymbol{\sigma}}{\partial \varepsilon}\right]_{\vartheta} \mathrm{d} \vartheta+\tau \int_{0}^{1}\left[\frac{\mathbf{D} \mathbf{u}_{\overline{\mathcal{S}}}}{\mathbf{D} \mathbf{x}_{b}} \frac{\mathrm{d} \boldsymbol{\eta}_{b}}{\mathrm{~d} t}\right]_{\vartheta} \mathrm{d} \vartheta+\varepsilon \int_{0}^{1}\left[\frac{\mathbf{D} \mathbf{u}_{\overline{\mathcal{S}}}}{\mathbf{D} \mathbf{x}_{b}} \frac{\mathbf{D} \boldsymbol{\eta}_{b}}{\mathbf{D c}}\right]_{\vartheta} \mathrm{d} \vartheta \mathfrak{g}(\alpha, b, \varepsilon)+ \\
+\varepsilon \mathbf{v}_{\overline{\mathscr{S}}}\left(\hat{\boldsymbol{\xi}}_{a}, \hat{\boldsymbol{\xi}}_{b}, \varepsilon\right) \equiv 0
\end{aligned}
$$

where $\hat{\xi}(t)=\boldsymbol{\xi}(t, \boldsymbol{C}(t, \varrho(\alpha, b, \varepsilon), \varepsilon), \varepsilon)$ and the meaning of the index $\vartheta$ may easily be found.

Inserting (4.37) into equations $\left(4.35^{\prime}\right)$ with indices from $\mathscr{S}$ we get

$$
\mathbf{w}_{\mathscr{S}}\left(\hat{\xi}_{a}, \hat{\xi}_{b}, \varepsilon\right)=0
$$


or, making use of the mean value theorem

$$
\mathbf{w}_{\mathscr{S}}\left(\hat{\xi}_{a}, \hat{\boldsymbol{\xi}}_{b}, \varepsilon\right) \equiv \mathbf{u}_{\mathscr{S}}\left(\hat{\boldsymbol{\eta}}(a), \hat{\boldsymbol{\eta}}\left(b_{0}\right)\right)+\tau \int_{0}^{1}\left[\frac{\mathbf{D} \mathbf{u}_{\mathscr{S}}}{\mathbf{D} \mathbf{c}_{\overline{\mathscr{V}}}} \frac{\partial \boldsymbol{\sigma}}{\partial b}\right]_{\mathscr{\vartheta}} \mathrm{d} \vartheta+
$$

$$
\begin{aligned}
+\varepsilon \int_{0}^{1}\left[\frac{\mathbf{D} \mathbf{u}_{\mathscr{S}}}{\mathbf{D} \boldsymbol{c}_{\overline{\mathcal{V}}}} \frac{\partial \boldsymbol{\sigma}}{\partial \varepsilon}\right]_{\vartheta} \mathrm{d} \vartheta+\tau \int_{0}^{1} & {\left[\frac{\mathbf{D} \mathbf{u}_{\mathscr{S}}}{\mathbf{D} \mathbf{x}_{b}} \frac{\mathrm{d} \boldsymbol{\eta}_{b}}{\mathrm{~d} t}\right]_{\vartheta} \mathrm{d} \vartheta+\varepsilon \int_{0}^{1}\left[\frac{\mathbf{D} \mathbf{u}_{\mathscr{S}}}{\mathbf{D} \mathbf{x}_{b}} \frac{\mathbf{D} \boldsymbol{\eta}_{b}}{\mathbf{D c}}\right]_{\vartheta} \mathrm{d} \vartheta \mathfrak{g}(\boldsymbol{\alpha}, b, \varepsilon)+} \\
& +\varepsilon \mathbf{v}_{\mathscr{S}}\left(\hat{\xi}_{a}, \hat{\xi}_{b}, \varepsilon\right)=0 .
\end{aligned}
$$

By (4.39) the system $\left(4.40^{\prime}\right)$ may be replaced for $\varepsilon \neq 0$ by an equivalent system

$$
\mathfrak{w}\left(\alpha, b_{0}+\tau, \varepsilon\right) \equiv \frac{1}{\varepsilon}\left[\mathbf{w}_{\mathscr{Y}}\left(\hat{\boldsymbol{\xi}}_{a}, \hat{\boldsymbol{\xi}}_{b}, \varepsilon\right)-\mathbf{T}(\alpha) \mathbf{w}_{\overline{\mathscr{S}}}\left(\boldsymbol{\xi}_{a}, \boldsymbol{\xi}_{b}, \varepsilon\right)\right]=0
$$

Letting $\varepsilon \rightarrow 0$ and taking into account (IIIe), (4.29), (4.29') and (4.30) the system (4.41) implies

$$
\begin{gathered}
\mathfrak{w}_{0}\left(\boldsymbol{\alpha}_{0}, b_{0}\right) \equiv \lim _{\varepsilon \rightarrow 0} \frac{\tau}{\varepsilon} \boldsymbol{\Psi}\left(b_{0}, \boldsymbol{\alpha}_{0}\right) \dot{\boldsymbol{\eta}}\left(b_{0}, \hat{\varrho}_{0}\left(\boldsymbol{\alpha}_{0}\right)\right)+ \\
+\int_{a}^{b_{0}} \boldsymbol{\Psi}\left(s, \boldsymbol{\alpha}_{0}\right) \boldsymbol{g}\left(\boldsymbol{\eta}\left(s, \hat{\varrho}_{0}\left(\boldsymbol{\alpha}_{0}\right)\right), 0\right) \mathrm{d} s+\hat{\boldsymbol{v}}_{0 \mathscr{S}}-\boldsymbol{T}\left(\boldsymbol{\alpha}_{0}\right) \hat{\boldsymbol{v}}_{0 \mathscr{S}}=0
\end{gathered}
$$

where

$$
\hat{\boldsymbol{v}}_{0}=\boldsymbol{v}\left(\boldsymbol{\eta}\left(a, \hat{\varrho}_{0}\left(\boldsymbol{\alpha}_{0}\right)\right), \boldsymbol{\eta}\left(b_{0}, \hat{\varrho}_{0}\left(\boldsymbol{\alpha}_{0}\right)\right), 0\right) \text {. }
$$

In the first place let us prove the existence of $\lim \tau / \varepsilon$. The existence of this limit will be deduced if we show that the system (4.26) has a real solution $v_{0}=v_{0}^{*}, \boldsymbol{\alpha}_{0}=\alpha_{0}^{*}$ and that $\boldsymbol{\Psi}\left(b_{0}, \boldsymbol{\alpha}_{0}^{*}\right) \dot{\boldsymbol{\eta}}\left(b_{0}, \hat{\varrho}_{0}\left(\boldsymbol{\alpha}_{0}^{*}\right)\right) \neq 0$. But the first fact is assured by the assumption (IVa) and the second by the assumption (IVc) since the column in the jacobian of this system corresponding to partial derivatives with respect to $v_{0}$ would otherwise be zero at the point $v_{0}=v_{0}^{*}, \boldsymbol{\alpha}_{0}=\boldsymbol{\alpha}_{0}^{*}$. Hence we may write $\tau(\varepsilon)=\varepsilon v(\varepsilon)$, where $v(\varepsilon)$ is continuous at the point $\varepsilon=0$.

Now substitute $\varepsilon v$ instead of $\tau$ into (4.41) and $v_{0}=v(0)$ instead of $\lim _{\varepsilon \rightarrow 0} \tau / \varepsilon$ into (4.42). Thus the latter system becomes precisely (4.26). Defining $\mathfrak{w}(\boldsymbol{\alpha}, b, 0)=\mathfrak{w}_{0}(\boldsymbol{\alpha}, b)$ the system (4.41) satisfies all assumptions of the implicit function theorem. Applying this theorem we deduce that there exists a unique solution $v=v^{*}(\varepsilon), \alpha_{0}=\alpha^{*}(\varepsilon)$ of (4.41) for sufficiently small $\varepsilon>0$ such that $\alpha^{*}(\varepsilon) \in \mathfrak{N}_{2}\left(\alpha_{0}^{*}, \delta_{2}\right)$ and $b^{*}(\varepsilon)=b_{0}+\varepsilon v^{*}(\varepsilon) \epsilon$ $\in \mathfrak{B}_{2}$. The existence of a unique solution $c^{*}(\varepsilon)=\varrho\left(\alpha^{*}(\varepsilon), b^{*}(\varepsilon), \varepsilon\right) \in C^{1}$ of $\left(4.35^{\prime}\right)$ then follows readily and this completes the proof of Theorem 4.1.

Corollary 4.5. Let an interval $\mathfrak{I}^{(0)}=\left\langle a, b_{0}\right\rangle$ be given. Consider the boundary value problem $(\kappa)$

$$
\dot{\mathbf{x}}=\mathbf{A x}+\varepsilon \mathbf{g}(\mathbf{x}, \varepsilon), \quad \mathbf{M} \mathbf{x}(a)+\mathbf{N} \mathbf{x}(b)+\varepsilon \mathbf{v}(\mathbf{x}(a), \mathbf{x}(b), \varepsilon)=0
$$

where the function $b$ with $b(0)=b_{0}$ has to be determined suitably. Let the following assumptions be fulfilled. 
(I) The same as (I) in Theorem 4.1.

(II) The same as (I) in Corollary 3.3.

(III) (a) Let the shortened boundary value problem $\left(\kappa_{0}\right)$

$$
\dot{\boldsymbol{y}}=\mathbf{A y}, \quad \mathbf{M} \mathbf{y}(a)+\mathbf{N} \mathbf{y}(b)=0
$$

have exactly $k$ linearly independent solutions. Denote $\tilde{\boldsymbol{\Phi}}(t) a n \times k$ matrix whose columns are formed by these solutions.

(b) Let $\tilde{\boldsymbol{\Phi}}(a) \gamma \in \mathfrak{P}, \tilde{\boldsymbol{\Phi}}\left(b_{0}\right) \gamma \in \mathfrak{Q}$ for $\gamma \in \mathfrak{G}, \mathbb{B}$ an open set in $E^{k}$.

(c) Put $\gamma_{i}=\lambda($ i an index from $\{1,2, \ldots, k\})$. Denote $\alpha=\gamma \dot{-}$. Let $\mathfrak{A}=E[\alpha \mid \alpha+$ $+\lambda \in$ (F)].

(IV) It may be shown that there exist a set $\mathscr{S} \subset \mathcal{N}, v(\mathscr{S})=k$, and a $k \times n$ constant matrix $\boldsymbol{T}$ such that for any fundamental matrix $\boldsymbol{\Phi ( t )}$ of solutions of (4.44) it holds

$$
(\mathbf{M} \Phi(a)+\mathbf{N} \Phi(b))_{\mathscr{S}} \equiv \boldsymbol{T}(\mathbf{M} \Phi(a)+\mathbf{N} \boldsymbol{\Phi}(b))_{\overline{\mathscr{S}}} .
$$

Further it may be shown that there exists a $k \times n$ matrix $\Psi(t)$ whose rows are formed by $k$ solutions of the boundary value problem $\left(\kappa_{0}^{*}\right)$

$$
\dot{\mathbf{w}}^{\prime}=-\mathbf{w}^{\prime} \mathbf{A}, \quad \mathbf{w}^{\prime}(a) \mathbf{P}^{\prime}+\mathbf{w}^{\prime}\left(b_{0}\right) \mathbf{Q}^{\prime}=0
$$

where $n \times n$ matrices $\mathbf{P}$ and $\mathbf{Q}$ satisfy conditions

$$
-\mathbf{M P}^{\prime}+\mathbf{N} \mathbf{Q}^{\prime}=0, \quad \chi(\mathbf{P}: \mathbf{Q})=n
$$

and which fulfils the condition

$$
\Psi(b)=\mathbf{N}_{\mathscr{S}}-\mathbf{T} \mathbf{N}_{\overline{\mathscr{I}}} .
$$

(a) Let the system

$$
\begin{aligned}
v_{0} \boldsymbol{\Psi}\left(b_{0}\right) \dot{\boldsymbol{\eta}}\left(b_{0}, \tilde{\boldsymbol{\Phi}}\left(b_{0}\right)\left(\boldsymbol{\alpha}_{0}\right.\right. & +\lambda))+\int_{a}^{b_{0}} \boldsymbol{\Psi}(s) \boldsymbol{g}\left(\boldsymbol{\eta}\left(s, \tilde{\boldsymbol{\Phi}}(s)\left(\boldsymbol{\alpha}_{0}+\lambda\right)\right), 0\right)+ \\
& +\mathbf{v}_{0 \mathscr{S}}-\boldsymbol{T}_{\mathbf{0}} \bar{\Phi}=0
\end{aligned}
$$

where $\mathbf{v}_{0}=\mathbf{v}\left(\tilde{\boldsymbol{\Phi}}(a)\left(\boldsymbol{\alpha}_{0}+\lambda\right), \tilde{\boldsymbol{\Phi}}(b)\left(\boldsymbol{\alpha}_{0}+\lambda\right), 0\right)$ have a real solution $v_{0}=v_{0}^{*}, \boldsymbol{\alpha}_{0}=\boldsymbol{\alpha}_{0}^{*}$.

(b) Let $\tilde{\boldsymbol{\Phi}}(a)\left(\alpha_{0}^{*}+\lambda\right) \in \mathfrak{G}$.

(c) Let $\dot{\Phi}_{l}(a)\left(\alpha_{0}^{*}+\lambda\right) \neq 0$.

(d) Let the jacobian of the system (4.45) be nonvanishing at the point $v_{0}=v_{0}^{*}$, $\alpha_{0}=\alpha_{0}^{*}$.

(V) Denote $\mathfrak{X}=E\left[\mathbf{x} \mid\left\|\mathbf{x}-\tilde{\boldsymbol{\Phi}}(t)\left(\alpha_{0}^{*}+\lambda\right)\right\|<\varrho, t \in \mathfrak{I}\right]$, $\varrho>0$. Let $\mathbf{g}(\mathbf{x}, \varepsilon) \in C^{1,1}$ for $\boldsymbol{x} \in \mathfrak{X}, \varepsilon \in \mathfrak{E}$.

Then there exists for sufficiently small $\varepsilon>0$ a unique function $b^{*}(\varepsilon)=b_{0}+$ $+\varepsilon v^{*}(\varepsilon), v^{*}(\varepsilon) \in C^{1}$ such that there exists a unique solution $\xi\left(t, c^{*}(\varepsilon), \varepsilon\right)$ of the problem $(\kappa)$ on the interval $\left\langle a, b^{*}(\varepsilon)\right\rangle$ with $\xi\left(a, c^{*}(\varepsilon), \varepsilon\right)=c^{*}(\varepsilon) \in C^{1}, c_{l}^{*}(\varepsilon)=$ $=\tilde{\boldsymbol{\Phi}}_{l}(a)\left(\alpha_{0}^{*}+\lambda\right)$ which tends to the solution $\Phi(t)\left(\alpha_{0}^{*}+\lambda\right)$ of the problem $\left(\kappa_{0}\right)$ as $\varepsilon \rightarrow 0$. 
Corollary 4.6. Consider the differential system (4.1). Let the following assumptions be fulfilled.

(I) The same as (III) (a) (b) (c) (e) in Theorem 4.1 with the exception that $a=0$, $b=\omega$ and that $\mathbf{u}$ has the particular form (representing periodic boundary conditions)

$$
\boldsymbol{u}(\boldsymbol{\eta}(0), \boldsymbol{\eta}(\omega))=\boldsymbol{\eta}(0)-\boldsymbol{\eta}(\varphi) .
$$

(II) Let the variational system

$$
\dot{\mathbf{z}}=\boldsymbol{f}_{\boldsymbol{x}}(\tilde{\boldsymbol{\eta}}(t)) \mathbf{z}
$$

have exactly $k$ linearly independent $\omega$-periodic solutions for $\gamma \in \mathbb{B}$.

Then the adjoint system $\dot{\boldsymbol{w}}^{\prime}=-\boldsymbol{w}^{\prime} \boldsymbol{f}_{\dot{\boldsymbol{x}}}(\hat{\boldsymbol{\eta}}(t))$ has also exactly $k$ linearly independent solutions for $\alpha+\lambda \in \mathcal{B}$. Denote $\Psi(t, \alpha)$ the $k \times n$ matrix whose rows are formed by them.

(IV) (a) Let the system

$$
v_{0} \boldsymbol{\Psi}\left(\omega, \boldsymbol{\alpha}_{0} \dot{\boldsymbol{\eta}}\left(\omega, \hat{\varrho}_{0}\left(\boldsymbol{\alpha}_{0}\right)\right)\right)+\int_{0}^{\omega} \boldsymbol{\Psi}\left(s, \boldsymbol{\alpha}_{0}\right) \boldsymbol{g}\left(\boldsymbol{\eta}\left(s, \hat{\varrho}_{0}\left(\boldsymbol{\alpha}_{0}\right)\right), 0\right) \mathrm{d} s=0
$$

have a real solution $v_{0}=v_{0}^{*}, \alpha_{0}=\alpha_{0}^{*}$.

(b) Let $\dot{\eta}_{l}\left(0, \hat{\varrho}_{0}\left(\alpha_{0}^{*}\right)\right) \neq 0$.

(c) Let the jacobian of the system (4.49) with respect to $v_{0}, \alpha_{0}$ be nonvanishing at the point $v_{0}=v_{0}^{*}, \boldsymbol{\alpha}_{0}=\boldsymbol{\alpha}_{0}^{*}$.

(V) The same as (VI) in Theorem 4.1.

Then there exists a unique function $\omega^{*}(\varepsilon)=\omega+\varepsilon v^{*}(\varepsilon), v^{*}(\varepsilon) \in C^{1}$ such that the system (4.1) has a unique $\omega^{*}(\varepsilon)$-periodic solution $\xi\left(t, c^{*}(\varepsilon), \varepsilon\right)$ with $\xi\left(0, c^{*}(\varepsilon), \varepsilon\right)=$ $=c^{*}(\varepsilon) \in C^{1}$ which tends to the $\omega$-periodic solution $\boldsymbol{\eta}\left(t, \hat{\varrho}_{0}\left(\alpha_{0}^{*}\right)\right)$ of the system $(4.3)$ as $\varepsilon \rightarrow 0$.

Remark 4.1. Let us prove that

$$
\Psi(t, \alpha) \dot{\eta}\left(t, \hat{\varrho}_{0}(\alpha)\right)=\text { const for } \alpha \in \mathfrak{A I}_{1} \text {. }
$$

Indeed, differentiating (4.48) with respect to $t$, we get

$$
-\boldsymbol{\Psi}(t, \alpha) \boldsymbol{f}_{\boldsymbol{x}}(t, \hat{\boldsymbol{\eta}}(t)) \dot{\hat{\boldsymbol{\eta}}}(t)+\boldsymbol{\Psi}(t, \alpha) \boldsymbol{f}_{\boldsymbol{x}}(t, \hat{\boldsymbol{\eta}}(t)) \dot{\hat{\boldsymbol{\eta}}}(t) \equiv 0 \text {. }
$$

Thus, we may write $\boldsymbol{\Psi}\left(a, \boldsymbol{\alpha}_{0}\right) \dot{\boldsymbol{\eta}}\left(a, \hat{\varrho}_{0}\left(\boldsymbol{\alpha}_{0}\right)\right)$ instead of $\boldsymbol{\Psi}\left(b_{0}, \boldsymbol{\alpha}_{0}\right) \dot{\boldsymbol{\eta}}\left(b_{0}, \hat{\varrho}_{0}\left(\boldsymbol{\alpha}_{0}\right)\right)$ in (4.26) and $\boldsymbol{\Psi}\left(0, \boldsymbol{\alpha}_{0}\right) \dot{\boldsymbol{\eta}}\left(0, \hat{\varrho}_{0}\left(\boldsymbol{\alpha}_{0}\right)\right)$ instead of $\boldsymbol{\Psi}\left(\omega, \boldsymbol{\alpha}_{0}\right) \dot{\boldsymbol{\eta}}\left(\omega, \hat{\varrho}_{0}\left(\boldsymbol{\alpha}_{0}\right)\right)$ in (4.47).

Now let us state the following theorem which corresponds to Theorem 3.1.

Theorem 4.2. Let an interval $\mathfrak{I}^{(0)}=\left\langle a, b_{0}\right\rangle$ be given. Consider the boundary value problem $(\alpha)$ given by (4.1), (4.2). Let the following assumptions be fulfilled.

(I), (II) The same as in Theorem 4.1.

(III) The same as in Theorem 4.1 with the exception that now $v(\mathscr{V})=k=1$ and with the the addition that $\dot{\boldsymbol{\eta}}_{\mathscr{V}}\left(a, \varrho_{0}(\lambda)\right) \neq 0$. 
(IV) The same as in Theorem 4.1.

(V) It may be shown that there exist an index $\mathscr{S} \subset \mathcal{N}$ and a constant matrix $T$ such that

$$
\frac{\mathrm{D} \hat{\mathbf{u}}_{\mathscr{S}}}{\mathrm{Dc}}-T \frac{\mathrm{D} \hat{\mathbf{u}}_{\bar{\varphi}}}{\mathrm{D} \boldsymbol{c}} \equiv 0
$$

Let

$$
\left(\frac{\mathbf{D} \hat{\mathbf{u}}_{\mathscr{S}}}{\mathbf{D} \boldsymbol{x}_{b}}-T \frac{\mathbf{D} \hat{\mathbf{u}}_{\overline{\mathcal{S}}}}{\mathbf{D} \boldsymbol{x}_{b}}\right) \frac{\mathrm{d} \hat{\boldsymbol{\eta}}_{b}}{\mathrm{~d} t} \neq 0 .
$$

(VI) The same as in Theorem 4.1 .

Then there exists for sufficiently small $\varepsilon>0$ a unique function $\tau^{*}(\varepsilon) \in C^{1}$ with $\tau^{*}(0)=0$ such that there exists a unique solution $\xi\left(t, c^{*}(\varepsilon), \varepsilon\right)$ of the problem $(\alpha)$ with $\xi\left(a, c^{*}(\varepsilon), \varepsilon\right)=c^{*}(\varepsilon) \in C^{1}, \mathbf{c}_{\mathscr{y}}^{*}(\varepsilon)=\lambda$ on the interval $\left\langle a, b_{0}+\tau^{*}(\varepsilon)\right\rangle$ which tends to the solution $\boldsymbol{\eta}\left(t, \varrho_{0}(\lambda)\right)$ of the problem $\left(\alpha_{0}^{\prime}\right)$ with $\boldsymbol{\eta}_{\mathfrak{r}}\left(a, \varrho_{0}(\lambda)\right)=\lambda$ on the interval $\left\langle a, b_{0}\right\rangle$ as $\varepsilon \rightarrow 0$.

The proof may be omitted since it is very similar to the proof of Theorem 4.1. (Notice that besides $n-1$ equations

$$
\mathbf{w}_{\overline{\mathscr{S}}}\left(\xi\left(a, \mathbf{c}_{\bar{\gamma}}+\lambda, \varepsilon\right), \boldsymbol{\xi}\left(b_{0}+\tau, \boldsymbol{c}_{\mathscr{r}}+\lambda, \varepsilon\right), \varepsilon\right)=0
$$

for $c_{\bar{V}}$ with the solution $c_{\bar{V}}=\sigma(\tau, \varepsilon) \in C^{2,1}$ we have the equation

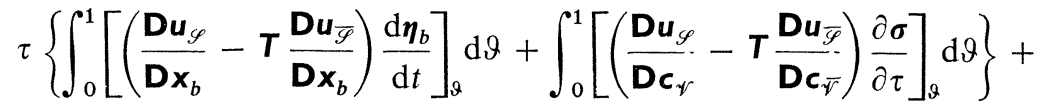

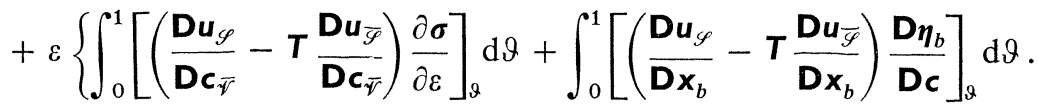

$$
\begin{aligned}
& \cdot \int_{a}^{b} \boldsymbol{\eta}_{c}^{-1}(s, \boldsymbol{C}(s, \varrho(\tau, \varepsilon), \varepsilon)) \mathbf{g}(\boldsymbol{\eta}(s, \boldsymbol{C}(s, \varrho(\tau, \varepsilon), \varepsilon)), \varepsilon) \mathrm{d} s+ \\
& \left.+\mathbf{v}_{\mathcal{S}}\left(\hat{\xi}_{a}, \hat{\xi}_{b}, \varepsilon\right)-T \mathbf{v}_{\overline{\mathcal{F}}}\left(\hat{\xi}_{a}, \hat{\xi}_{b}, \varepsilon\right)\right\}=0
\end{aligned}
$$

for $\tau$, which fulfils all assumptions of the implicit function theorem.)

A similar theorem for periodic boundary conditions was proved in [4] (Chap. 14, Th. 2.1) under less restrictive assumptions.

Theorem 4.3. Let an interval $\mathfrak{I}^{(0)}=\left\langle a, b^{(0)}\right\rangle$ be given. Consider the boundary value problem $(\alpha)$

$$
\begin{gathered}
\dot{\boldsymbol{x}}=\boldsymbol{f}(\mathbf{x})+\varepsilon \mathbf{g}(\mathbf{x}, \varepsilon), \\
\mathbf{w}(\mathbf{x}(a), \mathbf{x}(b(\varepsilon)), \varepsilon) \equiv \mathbf{u}\left(\mathbf{x}_{a}, \mathbf{x}_{b}\right)+\varepsilon \mathbf{v}\left(\mathbf{x}_{a}, \mathbf{x}_{b}, \varepsilon\right)=0
\end{gathered}
$$

where the function $b(\varepsilon)$ (fulfilling a condition with respect to $b^{(0)}$, see (IIIb)) has to be determined suitably. Let the following assumptions be fulfilled. 
(I), (II), (IIIa) and (VI) the same as in Theorem 4.1.

(III) (b) Let equations $\mathbf{u}(\boldsymbol{\eta}(a, \boldsymbol{c}), \boldsymbol{\eta}(b, \boldsymbol{c}))=0$ have a real solution $\boldsymbol{c}_{\bar{V}}=\boldsymbol{\sigma}_{0}\left(\boldsymbol{c}_{\boldsymbol{r}}\right)$, $b=\beta_{0}\left(\mathbf{c}_{\mathscr{V}}\right), \mathscr{V} \subset \mathscr{N}, v(\mathscr{V})=k, 2 \leqq k \leqq n$.

(c) Denote $c_{\gamma}=\gamma$, $\varrho_{0}(\gamma)=\gamma+\sigma_{0}(\gamma)$. Let $\sigma_{0}(\gamma) \in C^{2}, \beta_{0}(\gamma) \in C^{2}$ for $\gamma \in \mathbb{G}$, (B) an open set in $E^{k}$ such that $\mathfrak{D}=E\left[\mathbf{c} \mid \boldsymbol{c}=\varrho_{0}(\gamma), \gamma \in \mathfrak{G}\right] \subset \mathfrak{C}$.

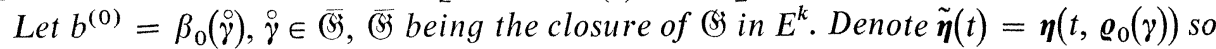
that $\tilde{\boldsymbol{\eta}}(t)$ is a $k$-parametric solution of the problem $\left(\alpha_{0}\right)$ given by (4.3), (4.4) on the interval $\left\langle a, \beta_{0}(\gamma)\right\rangle$.

(d) Let $\hat{\boldsymbol{\eta}}(a) \in \mathfrak{W}, \tilde{\boldsymbol{\eta}}\left(\beta_{0}(\gamma)\right) \in \mathfrak{D}$, for $\gamma \in(\mathfrak{S}$.

(e) Let $l \in \mathscr{V}$. Put $c_{l}=\lambda \in E\left[c_{l} \mid c_{l}=\gamma_{l}, \gamma \in\right.$ (S) $]$.

Denote $\alpha=\gamma-\lambda, \hat{\sigma}_{0}(\alpha)=\sigma_{0}(\alpha+\lambda), \hat{\varrho}_{0}(\alpha)=\varrho_{0}(\alpha+\lambda), \dot{\hat{\eta}}(t)=\eta\left(t, \hat{\varrho}_{0}(\alpha)\right)$. (Thus, $\eta(t)$ is a $(k-1)$-parametric solution of the problem $\left(\alpha_{0}\right)$ with $c_{l}=\lambda$.

(IV) Let the variational boundary value problem $(\mu)$

where

$$
\dot{\mathbf{z}}=\boldsymbol{f}_{\mathbf{x}}(\tilde{\boldsymbol{\eta}}(t)) \mathbf{z}, \frac{\mathrm{D} \tilde{\mathbf{u}}}{\mathrm{D} \mathbf{x}_{\boldsymbol{a}}} \mathbf{z}(a)+\frac{\mathrm{Du}}{\mathrm{Dx}_{\boldsymbol{b}}} \mathbf{z}(b)=0
$$

$$
\left(\frac{\mathbf{D} \tilde{u}}{\mathbf{D x}_{a}}: \frac{\mathbf{D} \tilde{u}}{\mathbf{D} \boldsymbol{x}_{b}}\right)=\left(\frac { \mathbf { D u } } { \mathbf { D } \mathbf { x } _ { \boldsymbol { a } } } \left(\tilde{\boldsymbol{\eta}}(a), \tilde{\boldsymbol{\eta}}\left(\beta_{0}(\gamma)\right): \frac{\mathbf{D u}}{\mathbf{D} \boldsymbol{x}_{\boldsymbol{b}}}\left(\tilde{\boldsymbol{\eta}}(a), \tilde{\boldsymbol{\eta}}\left(\beta_{0}(\gamma)\right)\right),\right.\right.
$$

have exactly $(k-1)$ linearly independent solutions on the interval $\left\langle a, \beta_{0}(\gamma)\right\rangle$ for $\gamma \in \mathbb{B}$.

(V) We shall show that there exist a set $\mathfrak{A}_{0}, \mathfrak{H}_{0}$ a domain in $E^{k-1}$ such that $E\left[\alpha+\lambda \mid \alpha \in \mathfrak{A}_{0}\right] \subset \mathfrak{G}$, a set $\mathscr{S} \subset \mathcal{N}, v(\mathscr{S})=k-1$ and $a(k-1) \times(n-k+1)$ matrix $\boldsymbol{T}(\alpha)$ such that

$$
\frac{\mathbf{D} \hat{\mathbf{u}}_{\mathscr{S}}}{\mathbf{D} \mathbf{c}}-\boldsymbol{T}(\alpha) \frac{\mathbf{D} \hat{\mathbf{u}}_{\overline{\mathscr{S}}}}{\mathbf{D} \mathbf{c}} \equiv 0, \quad \operatorname{det}\left(\frac{\mathbf{D} \hat{\mathbf{u}}}{\mathbf{D} \mathbf{c}}\right)_{\overline{\mathcal{S}} \overline{\mathcal{V}}} \neq 0,
$$

where

for $\alpha \in \mathfrak{A}_{0}$.

$$
\frac{\mathbf{D} \hat{u}}{\mathbf{D} \mathbf{c}}=\frac{\mathbf{D u}}{\mathbf{D} \boldsymbol{c}}\left(\hat{\boldsymbol{\eta}}(a), \hat{\boldsymbol{\eta}}\left(\hat{\beta}_{0}\right)\right)
$$

Further we shall show that there exists a $(k-1) \times n$ matrix $\Psi(t, \alpha)$ whose rows are formed by $k-1$ solutions of the boundary value problem $\left(\hat{\mu}^{*}\right)($ adjoint to $(\mu)$ in which $c_{l}=\lambda$ is put)

$$
\dot{\boldsymbol{w}}^{\prime}=-\boldsymbol{w}^{\prime} \boldsymbol{f}_{\mathbf{x}}(\hat{\boldsymbol{\eta}}(t)), \quad \mathbf{w}^{\prime}(a) \boldsymbol{P}^{\prime}(\alpha)+\mathbf{w}^{\prime}(b) \mathbf{Q}^{\prime}(\boldsymbol{\alpha})=0
$$

on the interval $\left\langle a, \hat{\beta}_{0}(\boldsymbol{\alpha})\right\rangle$ where $n \times n$ matrices $\mathbf{P}(\boldsymbol{\alpha})$ and $\mathbf{Q}(\boldsymbol{\alpha})$ satisfy conditions

$$
-\frac{\mathbf{D} \hat{u}}{\mathbf{D x}_{a}} \mathbf{P}^{\prime}(\alpha)+\frac{\mathbf{D} \hat{u}}{\mathbf{D} \mathbf{x}_{b}} \mathbf{Q}^{\prime}(\alpha) \equiv 0, \quad \chi(\boldsymbol{P}(\alpha): \mathbf{Q}(\alpha))=n
$$

for $\alpha \in \mathfrak{H}_{1}, \mathfrak{A}_{1}$ a domain in $\mathfrak{H}_{0}$, and which fulfils the condition

$$
\Psi\left(\hat{\beta}_{0}(\alpha), \alpha\right)=\frac{\mathbf{D} \hat{\mathbf{u}}_{\mathscr{S}}}{\mathbf{D} x_{b}}-\mathbf{T}(\alpha) \frac{\mathbf{D} \hat{\mathbf{u}}_{\overline{\mathscr{T}}}}{\mathbf{D} \boldsymbol{x}_{b}} .
$$


(a) Let the system

$$
\int_{a}^{\hat{\beta}_{0}\left(\alpha_{0}\right)} \boldsymbol{\Psi}\left(s, \boldsymbol{\alpha}_{0}\right) \boldsymbol{g}\left(\boldsymbol{\eta}\left(s, \hat{\varrho}_{0}\left(\boldsymbol{\alpha}_{0}\right)\right), 0\right) \mathrm{d} s+\hat{\mathbf{v}}_{0 \mathscr{S}}-\boldsymbol{T}\left(\boldsymbol{\alpha}_{0}\right) \hat{\mathbf{v}}_{0 \overline{\mathscr{S}}}=0
$$

where $\hat{\mathbf{v}}_{0}=\boldsymbol{v}\left(\boldsymbol{\eta}\left(a, \hat{\varrho}_{0}\left(\boldsymbol{\alpha}_{0}\right)\right), \boldsymbol{\eta}\left(\hat{\beta}_{0}\left(\boldsymbol{\alpha}_{0}\right), \hat{\varrho}_{0}\left(\boldsymbol{\alpha}_{0}\right)\right), 0\right)$ have a real solution $\boldsymbol{\alpha}_{0}=\boldsymbol{\alpha}_{0}^{*}$.

(b) Let $\alpha_{0}^{*} \in \mathfrak{U}_{1}$.

(c) Let $\dot{\eta}_{l}\left(a, c_{0}^{*}\right) \neq 0$, where $c_{0}^{*}=\hat{\varrho}_{0}\left(\alpha_{0}^{*}\right)$.

(d) Let the jacobian of the system (4.52) with respect to $\alpha_{0}$ be nonvanishing at the point $\alpha_{0}=\alpha_{0}^{*}$.

Then there exists for sufficiently small $\varepsilon>0$ a unique function $b^{*}(\varepsilon) \in C^{1}$ such that there exists a unique solution $\xi\left(t, c^{*}(\varepsilon), \varepsilon\right)$ of the problem $(\alpha)$ with $\xi\left(a, c^{*}(\varepsilon), \varepsilon\right)=$ $=c^{*}(\varepsilon) \in C^{1}, c_{l}^{*}(\varepsilon)=\lambda$ on the interval $\left\langle a, b^{*}(\varepsilon)\right\rangle$ which tends to the solution $\eta\left(t, c_{0}^{*}\right)$ of the problem $\left(\alpha_{0}\right)$ on the interval $\left\langle a, \hat{\beta}_{0}\left(\alpha_{0}^{*}\right)\right\rangle$ as $\varepsilon \rightarrow 0$.

Proof. By the same argument as in Theorem 4.1 we find that

$$
\chi\left(\frac{\mathbf{D} \tilde{u}}{\mathbf{D} \boldsymbol{c}}\right)=n-k+1 \text { for } \gamma \in \mathbb{B} \text {. }
$$

Let us show that

$$
\chi\left(\frac{\mathbf{D} \tilde{\mathbf{u}}}{\mathbf{D c}}: \frac{\mathrm{d} \tilde{\mathbf{u}}}{\mathrm{d} b}\right)=n-k+1 .
$$

In fact, the rank of the matrix in question obviously cannot be less than $n-k+1$. On the other hand writing the identity

$$
\boldsymbol{u}\left(\boldsymbol{\eta}\left(a, \varrho_{0}(\gamma)\right), \boldsymbol{\eta}\left(\beta_{0}(\gamma), \varrho_{0}(\gamma)\right)\right) \equiv 0
$$

and differentiating it with respect to $\gamma$ we get

$$
\frac{\mathbf{D} \tilde{u}}{\mathbf{D c}} \frac{\mathbf{D} \varrho_{0}}{\mathbf{D} \gamma}+\frac{\mathrm{d} \tilde{\boldsymbol{u}}}{\mathrm{d} b} \frac{\mathbf{D} \beta_{0}}{\mathbf{D} \gamma} \equiv 0
$$

or in a somewhat different form

$$
\left(\frac{\mathbf{D} \tilde{\mathbf{u}}}{\mathbf{D c}}: \frac{\mathrm{d} \tilde{\boldsymbol{u}}}{\mathrm{d} b}\right) \frac{\mathbf{D}\left(\varrho_{0}, \beta_{0}\right)}{\mathbf{D} \boldsymbol{\gamma}} \equiv 0
$$

Since evidently

$$
\chi\left(\frac{\left.\mathbf{D}_{0}, \beta_{0}\right)}{\mathbf{D} \gamma}\right)=k
$$

by the known theorem of algebra

$$
\chi\left(\frac{\mathbf{D} \tilde{\mathbf{u}}}{\mathbf{D} \mathbf{c}}: \frac{\mathrm{d} \tilde{\boldsymbol{u}}}{\mathrm{d} b}\right) \leqq n-k+1
$$

whence $(4.54)$ follows readily. 
Hence there exist sets $\mathscr{S} \subset \mathscr{N}, \mathscr{V}_{1} \subset \mathcal{N}, v(\mathscr{S})=k-1, v\left(\mathscr{V}_{1}\right)=k$ and $\mathscr{G}_{0}, \mathfrak{B S}_{0}$ a domain in $\mathfrak{G}$, such that for $\gamma \in \mathfrak{G}_{0}$

$$
\chi\left(\frac{\mathbf{D} \tilde{\mathbf{u}}_{\overline{\mathscr{Y}}}}{\mathbf{D}_{\overline{\mathscr{V}}_{1}}}: \frac{\mathrm{d} \tilde{\mathbf{u}}_{\overline{\mathscr{Y}}}}{\mathrm{d} b}\right)=n-k+1 .
$$

Let us now show that in particular

$$
\operatorname{det} \boldsymbol{V}(\gamma)=\operatorname{det}\left(\frac{\mathbf{D u}_{\overline{\mathscr{y}}}}{\mathbf{D c}_{\overline{\mathcal{V}}}}: \frac{\mathrm{d} \tilde{\mathbf{u}}_{\overline{\mathscr{y}}}}{\mathrm{d} b}\right) \neq 0 .
$$

In fact, taking into account that

$$
\mathbf{u}_{\overline{\mathcal{S}}}\left(\boldsymbol{\eta}\left(a, \varrho_{0}(\gamma)\right), \boldsymbol{\eta}\left(\beta_{0}(\gamma), \varrho_{0}(\gamma)\right)\right) \equiv 0
$$

for $\gamma \in \mathcal{B}$ and differentiating this identity, we get

$$
\left(\frac{\mathbf{D} \tilde{u}}{\mathbf{D} \boldsymbol{c}_{\mathscr{\gamma}}}: \frac{\mathrm{d} \tilde{\mathbf{u}}}{\mathrm{d} b}\right)_{\mathscr{S}} \frac{\mathbf{D}\left(\sigma_{0}, \beta_{0}\right)}{\mathbf{D} \boldsymbol{\gamma}} \equiv 0 .
$$

Clearly if for some point $\dot{\gamma} \in \mathfrak{S}_{0}$

$$
\chi(\mathbf{V}(\gamma)) \leqq n-k
$$

then there would exist an index $j \in \overline{\mathscr{S}}$ and a $1 \times(n-k)$ matrix $\boldsymbol{M}(\gamma)$ such that (denoting $\mathscr{J}$ the complement of $j$ with respect to $\overline{\mathscr{S}}$ )

$$
\boldsymbol{V}_{j}(\stackrel{\circ}{\gamma})-\mathbf{M}(\stackrel{\circ}{\gamma}) \boldsymbol{V}_{\mathscr{g}}(\stackrel{\circ}{\gamma})=0 \text {. }
$$

Then by (4.56)

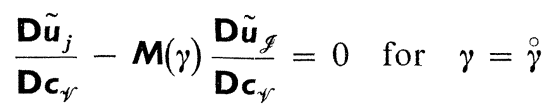

which together with (4.59) contradicts (4.57).

The relations (4.53), (4.54) and (4.58) evidently continue hold if we put $c_{l}=\lambda \epsilon$ $\in E\left[c_{l} \mid c_{l}=\eta_{l}\left(a, \varrho_{0}(\gamma)\right), \gamma \in(\mathfrak{S})\right]$. Thus there exists a $(k-1) \times(n-k+1)$ matrix $T(\alpha)$ such that

$$
\left(\frac{\mathbf{D} \hat{\mathbf{u}}_{\mathscr{S}}}{\mathbf{D} \mathbf{c}}: \frac{\mathrm{d} \hat{\mathbf{u}}_{\mathscr{S}}}{\mathrm{d} b}\right)-\mathbf{T}(\alpha)\left(\frac{\mathbf{D} \hat{\mathbf{u}}_{\overline{\mathscr{Y}}}}{\mathbf{D c}}: \frac{\mathrm{d} \hat{\mathbf{u}}_{\overline{\mathscr{Y}}}}{\mathrm{d} b}\right) \equiv 0
$$

for $\alpha \in \mathfrak{A}_{0}=E\left[\alpha \mid \alpha+\lambda \in \mathfrak{G}_{0}\right]$, whence it follows in particular

$$
\frac{\mathbf{D} \hat{\mathbf{u}}_{\mathscr{\varphi}}}{\mathbf{D} \boldsymbol{c}_{\overline{\mathcal{V}}}}-\mathbf{T}(\boldsymbol{\alpha}) \frac{\mathbf{D} \hat{\mathbf{u}}_{\overline{\mathscr{Y}}}}{\mathbf{D} \boldsymbol{c}_{\overline{\mathcal{V}}}} \equiv 0, \frac{\mathrm{d} \hat{\mathbf{u}}_{\mathscr{P}}}{\mathrm{d} b}-\mathbf{T}(\boldsymbol{\alpha}) \frac{\mathrm{d} \mathbf{u}_{\overline{\mathscr{Y}}}}{\mathrm{d} b} \equiv 0,
$$

and

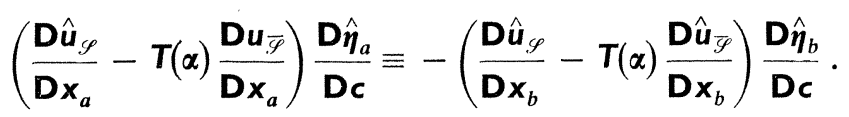

Quite analogously as in previous cases we may prove that the adjoint boundary value problem $\left(\hat{\mu}^{*}\right)$ is defined and has exactly $k-1$ linearly independent solutions for 
$\alpha \in \mathfrak{A}_{1}, \mathfrak{A}_{1}$ a domain in $\mathfrak{A}_{0}$, and that there exists the matrix $\Psi(t, \alpha)$ with properties listed in (V) and namely that

$$
\boldsymbol{\Psi}(t, \alpha)=\left(\frac{\mathbf{D} \hat{\mathbf{u}}_{\mathscr{S}}}{\mathbf{D} \boldsymbol{x}_{b}}-\mathbf{T}(\alpha) \frac{\mathbf{D} \hat{\mathbf{u}}_{\overline{\mathscr{P}}}}{\mathbf{D} \boldsymbol{x}_{\boldsymbol{b}}}\right) \frac{\mathbf{D} \hat{\boldsymbol{\eta}}}{\mathbf{D} \mathbf{c}}\left(\hat{\beta}_{0}(\alpha)\right)\left(\frac{\mathbf{D} \hat{\boldsymbol{\eta}}}{\mathbf{D c}}(t)\right)^{-1}
$$

(to prove it we make use of $\left(4.60^{\prime \prime}\right)$ ).

Now equations (4.52) are completely defined for $\alpha \in \mathfrak{A}_{1}$ and their solution $\alpha_{0}=\alpha_{0}^{*}$ with properties (Vb, c, d) may be found.

As in Theorem 4.1 we may require that the solution $\xi\left(t, c^{*}(\varepsilon), \varepsilon\right)$ of the problem $(\alpha)$ fulfils the condition

$$
c_{l}^{*}(\varepsilon)=\lambda .
$$

By the same argument as in Theorem 4.1 there exists a neighborhood $\mathfrak{D}_{1}\left(c_{0}^{*}, \delta_{1}\right), \mathbf{c}_{0}^{*}=$ $=\hat{\varrho}_{0}\left(\alpha_{0}^{*}\right)$ and sets $\mathfrak{B}_{1}=\left\langle b_{0}^{*}-\pi_{1}, b_{0}^{*}+\pi_{1}\right\rangle, b_{0}^{*}=\hat{\beta}_{0}\left(\alpha_{0}^{*}\right), \mathfrak{F}_{1}=\left\langle 0, \varepsilon_{1}\right\rangle, 0<\varepsilon_{1} \leqq$ $\leqq \varepsilon_{0}$ such that (1) every solution $\xi(t, c, \varepsilon)$ of (4.1) where $\varepsilon \in \mathfrak{F}_{1}$ starting on $\mathfrak{D}_{1}$ stays in $\mathfrak{X}$ for $t \in \mathfrak{I}_{0}$ and $\xi(b, c, \varepsilon) \in \mathfrak{D}_{\text {f }}$ for $b \in \mathfrak{B}_{1}, c \in \mathfrak{D}_{1}, \varepsilon \in \mathfrak{F}_{1},(2)$ it may be written in the form (4.37) where $\boldsymbol{C}(t, c, \varepsilon)$ is a solution of (4.38) and finally (3) by (4.58)

$$
\operatorname{det}\left(\frac{\mathbf{D} \boldsymbol{w}_{\mathscr{S}}}{\mathbf{D} \mathbf{c}_{\bar{V}}}\left(\xi_{a}, \xi_{b}, \varepsilon\right): \frac{\mathrm{d} \boldsymbol{w}_{\overline{\mathscr{Y}}}}{\mathrm{d} b}\left(\boldsymbol{\xi}_{a}, \boldsymbol{\xi}_{b}, \varepsilon\right)\right) \neq 0
$$

for $b \in \mathfrak{B}_{1}, c \in \mathfrak{S}_{1}$ and $\varepsilon \in \mathfrak{E}_{1}$.

A solution $\xi(t, c, \varepsilon)$ of (4.1) is a solution of the problem $(\alpha)$ if and only if

$$
\mathbf{w}(\xi(a, c, \varepsilon), \xi(b, c, \varepsilon), \varepsilon)=0 .
$$

Inserting (4.62) into (4.64) we get the system of equations (denoting $\mathscr{L}$ the complement of $l$ with respect to $\mathscr{V})$

$$
\mathbf{w}\left(\xi\left(a, \mathbf{c}_{\bar{\gamma}}+\mathbf{c}_{\mathscr{L}}+\lambda, \varepsilon\right), \boldsymbol{\xi}\left(b, \boldsymbol{c}_{\bar{\gamma}}+c_{\mathscr{L}}+\lambda, \varepsilon\right), \varepsilon\right)=0
$$

for $n$ unknowns $c_{\bar{Y}}, c_{\mathscr{L}}$ and $b$.

Leaving equations (4.65) with indices from $\overline{\mathscr{S}}$ unchanged we have

$$
\mathbf{w}_{\overline{\mathscr{S}}}\left(\xi\left(a, \boldsymbol{c}_{\bar{V}}+\mathbf{c}_{\mathscr{L}}+\lambda, \varepsilon\right), \boldsymbol{\xi}\left(b, \boldsymbol{c}_{\bar{V}}+\boldsymbol{c}_{\mathscr{L}}+\lambda, \varepsilon\right), \varepsilon\right)=0 .
$$

By (4.63) and by the implicit function theorem there exist a neighborhood $\mathfrak{R}_{2}\left(\alpha_{0}^{*}, \delta_{2}\right)$ and a set $\mathfrak{E}_{2}=\left\langle 0, \varepsilon_{2}\right\rangle, 0<\varepsilon_{2} \leqq \varepsilon_{1}$ such that for $\boldsymbol{c}_{\mathscr{L}} \in \mathfrak{N}_{2}\left(\alpha_{0}^{*}, \delta_{2}\right)$ and $\varepsilon \in \mathfrak{E}_{2}$ there exists a unique solution

$$
\mathbf{c}_{\bar{V}}=\boldsymbol{\sigma}\left(\boldsymbol{c}_{\mathscr{L}}, \varepsilon\right), \quad b=\beta\left(\boldsymbol{c}_{\mathscr{L}}, \varepsilon\right)
$$

of (4.66) such that (denoting again $\left.\mathbf{c}_{\mathscr{L}}=\alpha, \varrho(\alpha, \varepsilon)=\sigma(\alpha, \varepsilon)+\alpha+\lambda\right), \sigma(\alpha, \varepsilon) \in C^{2,1}$, $\beta(\alpha, \varepsilon) \in C^{2,1}, \boldsymbol{\sigma}(\alpha, 0)=\hat{\boldsymbol{\sigma}}_{0}(\alpha), \beta(\alpha, 0)=\hat{\beta}_{0}(\alpha)$ and $\varrho(\alpha, \varepsilon) \in \mathfrak{D}_{1}\left(\alpha_{0}^{*}, \delta_{1}\right), \beta(\alpha, \varepsilon) \in \mathfrak{B}_{1}$. Inserting the solution (4.67) into (4.66) we get the identity

$$
\mathbf{w}_{\overline{\mathscr{J}}}(\hat{\xi}(a), \hat{\xi}(\beta(\alpha, \varepsilon)), \varepsilon) \equiv 0
$$


where $\hat{\xi}(t)=\xi(t, \varrho(\alpha, \varepsilon), \varepsilon)$ or making use of the mean value theorem and denoting

$$
\mathfrak{g}(\boldsymbol{\alpha}, \varepsilon)=\int_{a}^{\beta(\alpha, \varepsilon)} \boldsymbol{\eta}_{c}^{-1}(s, \boldsymbol{C}(s, \varrho(\boldsymbol{\alpha}, \varepsilon), \varepsilon)) \mathbf{g}(\boldsymbol{\eta}(s, \boldsymbol{C}(s, \varrho(\boldsymbol{\alpha}, \varepsilon), \varepsilon), \varepsilon)) \mathrm{d} s
$$

we have

$$
\begin{gathered}
\boldsymbol{w}_{\overline{\mathscr{S}}}(\hat{\boldsymbol{\xi}}(a), \hat{\boldsymbol{\xi}}(\beta(\boldsymbol{\alpha}, \varepsilon)), \varepsilon) \equiv \mathbf{u}_{\overline{\mathscr{S}}}\left(\hat{\boldsymbol{\eta}}(a), \hat{\boldsymbol{\eta}}\left(\hat{\beta}_{0}\right)\right)+ \\
+\varepsilon \int_{0}^{1}\left[\frac{\mathbf{D} \mathbf{u}_{\overline{\mathscr{S}}}}{\mathbf{D} \boldsymbol{c}_{\overline{\mathcal{V}}}} \frac{\partial \boldsymbol{\sigma}}{\partial \varepsilon}\right]_{\vartheta} \mathrm{d} \vartheta+\varepsilon \int_{0}^{1}\left[\frac{\mathbf{D} \mathbf{u}_{\overline{\mathscr{S}}}}{\mathbf{D} \boldsymbol{\eta}_{b}} \frac{\mathbf{D} \boldsymbol{b}}{\mathbf{D} \boldsymbol{b}}\right]_{\vartheta} \mathrm{d} \vartheta \mathfrak{g}(\boldsymbol{\alpha}, \varepsilon)+ \\
+\varepsilon \int_{0}^{1}\left[\frac{\mathrm{d} \mathbf{u}_{\overline{\mathscr{S}}}}{\mathrm{d} b} \frac{\partial \beta}{\partial \varepsilon}\right]_{\vartheta} \mathrm{d} \vartheta+\varepsilon \mathbf{v}_{\overline{\mathscr{S}}}(\hat{\boldsymbol{\xi}}(a), \hat{\boldsymbol{\xi}}(\beta(\boldsymbol{\alpha}, \varepsilon)), \varepsilon) \equiv 0
\end{gathered}
$$

where the meaning of the index $\vartheta$ may-easily be found in each case.

Inserting the solution (4.67) into equations (4.65) with indices from $\mathscr{S}$ we get

$$
\mathbf{w}_{\mathscr{S}}(\hat{\xi}(a), \hat{\xi}(\beta(\alpha, \varepsilon)), \varepsilon)=0
$$

or again making use of the mean value theorem

$$
\begin{gathered}
\boldsymbol{w}_{\mathscr{S}}(\hat{\boldsymbol{\xi}}(a), \hat{\boldsymbol{\xi}}(\beta(\boldsymbol{\alpha}, \varepsilon)), \varepsilon) \equiv \boldsymbol{u}\left(\hat{\boldsymbol{\eta}}(a), \hat{\boldsymbol{\eta}}\left(\hat{\beta}_{0}(\boldsymbol{\alpha})\right)\right)+ \\
+\varepsilon \int_{0}^{1}\left[\frac{\mathbf{D} \boldsymbol{u}_{\mathscr{S}}}{\mathbf{D}_{\overline{\mathscr{V}}}} \frac{\partial \boldsymbol{\sigma}}{\partial \varepsilon}\right]_{\vartheta} \mathrm{d} \vartheta+\varepsilon \int_{0}^{1}\left[\frac{\mathbf{D} \mathbf{u}_{\mathscr{Y}}}{\mathbf{D} \boldsymbol{x}_{\boldsymbol{b}}} \frac{\mathbf{D} \boldsymbol{\eta}_{b}}{\mathbf{D c}}\right]_{\vartheta} \mathrm{d} \vartheta \mathfrak{g}(\boldsymbol{\alpha}, \varepsilon)+ \\
+\varepsilon \int_{0}^{1}\left[\frac{\mathrm{d} \mathbf{u}_{\mathscr{S}} \partial \beta}{\mathrm{d} b}\right]_{\vartheta} \mathrm{d} \vartheta+\varepsilon \mathbf{v}_{\mathscr{S}}(\hat{\boldsymbol{\xi}}(a), \hat{\boldsymbol{\xi}}(\beta(\boldsymbol{\alpha}, \varepsilon)), \varepsilon)=0 .
\end{gathered}
$$

By (4.68) the system $\left(4.70^{\prime}\right)$ may be replaced for $\varepsilon \neq 0$ by the equivalent system

$$
\mathfrak{w}(\boldsymbol{\alpha}, \varepsilon) \equiv \frac{1}{\varepsilon}\left[\mathbf{w}_{\mathscr{S}}(\hat{\xi}(a), \hat{\xi}(\beta), \varepsilon)-\mathbf{T}(\alpha) \boldsymbol{w}_{\overline{\mathscr{S}}}(\hat{\boldsymbol{\xi}}(a), \xi(\beta), \varepsilon)\right]=0 .
$$

Letting $\varepsilon \rightarrow 0$ and taking into account (IIId), $\left(4.60^{\prime}\right)$ and (4.61) we get the system

$$
\mathfrak{w}_{0}\left(\boldsymbol{\alpha}_{0}\right) \equiv \int_{a}^{\hat{\beta}_{0}\left(\alpha_{0}\right)} \boldsymbol{\Psi}\left(s, \boldsymbol{\alpha}_{0}\right) \boldsymbol{g}\left(\boldsymbol{\eta}\left(s, \hat{\varrho}_{0}\left(\boldsymbol{\alpha}_{0}\right)\right), 0\right) \mathrm{d} s+\hat{\boldsymbol{v}}_{0 \mathscr{S}}-\boldsymbol{T}\left(\boldsymbol{\alpha}_{0}\right) \hat{\boldsymbol{v}}_{0 \overline{\mathscr{T}}}=0
$$

where $\hat{\boldsymbol{v}}_{0}=\mathbf{v}\left(\boldsymbol{\eta}\left(a, \hat{\varrho}_{0}\left(\boldsymbol{\alpha}_{0}\right)\right), \boldsymbol{\eta}\left(\hat{\beta}_{0}\left(\boldsymbol{\alpha}_{0}\right), \hat{\varrho}_{0}\left(\boldsymbol{\alpha}_{0}\right)\right), 0\right)$, which is identical with the system (4.52). Putting $\mathfrak{w}(\alpha, 0)=\mathfrak{w}_{0}(\alpha)$ it may be easily verified that by (II), (III) and (VI) $\mathfrak{w}(\alpha, \varepsilon) \in C^{1,1}$ on $\mathfrak{N}_{2}\left(\alpha_{0}^{*}, \delta_{2}\right) \times \mathcal{F}_{2}$. Hence taking further into account $(\mathrm{Vb}, \mathrm{c}, \mathrm{d})$ all assumptions of the implicit function theorem are fulfilled. Applying this theorem we deduce the existence of a unique solution $\alpha=\alpha^{*}(\varepsilon) \in C^{1}$ of $(4.70)$ such that $\alpha^{*}(0)=$ $=\alpha_{0}^{*}, \alpha^{*}(\varepsilon) \in \mathfrak{N}_{2}\left(\alpha_{0}^{*}, \delta_{2}\right)$ for sufficiently small $\varepsilon>0$ and that $\sigma\left(\alpha^{*}(\varepsilon), \varepsilon\right) \in C^{1}, b^{*}(\varepsilon)=$ $=\boldsymbol{\beta}\left(\boldsymbol{\alpha}^{*}(\varepsilon), \varepsilon\right) \in C^{1}$ is a solution of $(4.64)$ such that $\boldsymbol{c}^{*}(\varepsilon)=\varrho\left(\alpha^{*}(\varepsilon), \varepsilon\right) \in \coprod_{1}\left(\mathbf{c}_{0}^{*}, \delta_{1}\right)$, $b^{*}(\varepsilon) \in \mathfrak{B}_{1}$. Thus by the above considerations $\xi\left(t, c^{*}(\varepsilon), \varepsilon\right)$ is a solution of the problem $(\alpha)$ on the interval $\left\langle a, b^{*}(\varepsilon)\right\rangle$, which completes the proof of Theorem 4.3.

Corollary 4.7. Consider the system 4.1. Let the following assumptions be fulfilled.

(I) The same as (IIIa, b, c, e) in Theorem 4.3 with the exception that $a=0$, 
$b^{(0)}=\omega^{(0)}, \omega=\omega_{0}(\gamma)$ and the function $\boldsymbol{u}$ (representing the periodic boundary condition) has the particular form

$$
\boldsymbol{u}(\boldsymbol{\eta}(0), \boldsymbol{\eta}(\omega))=\boldsymbol{\eta}(0)-\boldsymbol{\eta}(\omega) .
$$

(II) Let the variational differential system

$$
\dot{\mathbf{z}}=\boldsymbol{f}_{\mathbf{x}}(\tilde{\boldsymbol{\eta}}(t)) \mathbf{z}
$$

have exactly $k-1$ linearly independent $\omega_{0}(\gamma)$-periodic solutions for $\gamma \in \mathfrak{G}$.

(III) It may be shown that there exists a $(k-1) \times n$ matrix $\Psi(t, \alpha)$ whose rows are formed by $k-1$ linearly independent $\hat{\omega}_{0}(\alpha)$-periodic solutions of the adjoint differential system

$$
\dot{w}^{\prime}=-w^{\prime} f_{x}(\hat{\eta}(t))
$$

where $\alpha \in \mathfrak{A}=E[\alpha \mid \alpha+\lambda \in \mathfrak{F}]$.

(a) Let the system

$$
\int_{0}^{\hat{\omega}_{0}\left(\alpha_{0}\right)} \boldsymbol{\Psi}\left(s, \boldsymbol{\alpha}_{0}\right) \mathbf{g}\left(\boldsymbol{\eta}\left(s, \hat{\varrho}_{0}\left(\boldsymbol{\alpha}_{0}\right)\right), 0\right) \mathrm{d} s=0
$$

have a real solution $\alpha_{0}=\alpha_{0}^{*}$.

(b) Let $\alpha_{0}^{*} \in \mathfrak{I}$.

(c) Let $\dot{\boldsymbol{\eta}}_{l}\left(0, \hat{\varrho}_{0}\left(\alpha_{0}^{*}\right)\right) \neq 0$.

(d) Let the jacobian of the system (4) with respect to $\alpha_{0}$ be nonvanishing at the point $\alpha_{0}=\alpha_{0}^{*}$.

(IV) The same as (VI) in Theorem 4.2.

Then there exists for sufficiently small $\varepsilon>0$ a unique $\omega^{*}(\varepsilon)$-periodic solution $\xi\left(t, c^{*}(\varepsilon), \varepsilon\right)$ of $(4.1)$ with $\xi\left(0, c^{*}(\varepsilon), \varepsilon\right)=c^{*}(\varepsilon) \in C^{1}, c_{l}^{*}(\varepsilon)=\lambda, \omega^{*}(\varepsilon) \in C^{1}$, which tends to the $\omega_{0}\left(\alpha_{0}^{*}\right)$-periodic solution $\boldsymbol{\eta}\left(t, \varrho_{0}\left(\alpha_{0}^{*}\right)\right)$ of $(4.3)$ as $\varepsilon \rightarrow 0$.

Remark 4.2. In Theorem 4.3 and Corollary 4.5 we may restrict ourselves to the case that $2 \leqq k \leqq n$. In fact, if $k=1$ then the unique parameter must evidently be equivalent to the parameter $\delta$ which may be added to the variable $t$. This implies that the whole one-parameter family exists on the same interval $\left\langle a, b_{0}\right\rangle$.

Remark 4,3. Remarks analogous to Remarks $3.1,3.3$ and 3.4 with respect to Theorems 4.1, 4.2 and 4.3 might be stated.

\section{Bibliography}

[1] E. A. Coddington, N. Levinson: Theory of ordinary differential equations. New York 1955.

[2] H. Poincaré: Les méthodes nouvelles de la mécanique célecte t. I. Paris 1892.

[3] И. Г. Малкин: Методы Ляпунова и Пуанкаре в теории нелинейных колебаний. Москва, 1949.

[4] И. Г. Малкин: Некоторые задачи теории нелинейных колебаний. Москва, 1956. 
[5] E. Bounizky: Sur la fonction de Green des équations différentielles linéaires ordinaires. Journal de Math. (6), 5 (1909), 65-125.

[6] O. Vejvoda: Perturbed boundary-value problems and their approximate solution. Proc. Rome Symp. num. treatment etc., Basel 1961, pp. 37-41.

\title{
Резюме
}

\section{ВОЗМУЩЕННЫЕ НЕЛИНЕЙНЫЕ КРАЕВЫЕ ЗАДАЧИ}

\author{
ОТТО ВЕЙВОДА (Otto Vejvoda), Прага
}

В первом параграфе приведены те теоремы из теории линейных краевых задач, которые используются в дальнейшем.

Во втором параграфе приводятся теоремы о методе вариации постоянных и о неявных функциях. Эти две теоремы являются основными средствами для изучения следующих вопросов.

В третьем параграфе рассматривается краевая задача $(v)$ :

$$
\begin{gathered}
\dot{\boldsymbol{x}}=\boldsymbol{f}(t, \mathbf{x})+\varepsilon \mathbf{g}(t, \mathbf{x}, \varepsilon), \\
\boldsymbol{w}\left(\boldsymbol{x}_{a}, \boldsymbol{x}_{b}, \varepsilon\right) \equiv \boldsymbol{u}\left(\boldsymbol{x}_{a}, \boldsymbol{x}_{b}\right)+\varepsilon \boldsymbol{v}\left(\boldsymbol{x}_{a}, \boldsymbol{x}_{b}, \varepsilon\right)=0,
\end{gathered}
$$

где $\boldsymbol{f}, \boldsymbol{g}, \boldsymbol{u}, \boldsymbol{v}, \boldsymbol{w}-n$-мерные векторы.

Предполагается, что решения укороченной (,предельной“) краевой задачи $\left(v_{0}\right)$

$$
\begin{gathered}
\dot{y}=f(t, y), \\
u\left(y_{a}, y_{b}\right)=0
\end{gathered}
$$

известны, и отыскиваются только те решения задачи $(v)$, которые стремятся для $\varepsilon \rightarrow 0$ к некоторому решению задачи $\left(v_{0}\right)$.

Важную роль в дальнейшем играет краевая задача „в вариациях“ $(\lambda)$ :

$$
\begin{gathered}
\dot{\mathbf{z}}=\frac{\mathbf{D} \boldsymbol{f}}{\mathbf{D} \mathbf{x}}(t, \tilde{\boldsymbol{\eta}}(t)) \mathbf{z}, \\
\frac{\mathbf{D u}}{\mathbf{D} \mathbf{x}_{\boldsymbol{a}}}\left(\tilde{\boldsymbol{\eta}}_{a}, \tilde{\boldsymbol{\eta}}_{b}\right) \mathbf{z}(a)+\frac{\mathbf{D u}}{\mathbf{D} \mathbf{x}_{\boldsymbol{b}}}\left(\tilde{\boldsymbol{\eta}}_{a}, \tilde{\boldsymbol{\eta}}_{b}\right) \mathbf{z}(b)=0,
\end{gathered}
$$

где $\tilde{\boldsymbol{\eta}}(t)=\boldsymbol{\eta}\left(t, \varrho_{0}(\gamma)\right)$, общее решение краевой задачи $\left(v_{0}\right)$, причем $\boldsymbol{\eta}(t, \boldsymbol{c})$, общее решение системы (3.3) с начальным значением $\boldsymbol{\eta}(\mathrm{a}, \boldsymbol{c})=c$ и $\varrho_{0}(\gamma)$, решение системы (3.4), зависящее от $k$ параметров $\gamma=\left(\gamma_{1}, \gamma_{2}, \ldots, \gamma_{k}\right)$. Как известно, если $\chi\left(\frac{\mathbf{D u}}{\mathbf{D} \mathbf{x}_{a}}\left(\tilde{\boldsymbol{\eta}}_{a}, \tilde{\boldsymbol{\eta}}_{b}\right): \frac{\mathbf{D u}}{\mathbf{D} \mathbf{x}_{b}}\left(\tilde{\boldsymbol{\eta}}_{a}, \tilde{\boldsymbol{\eta}}_{b}\right)\right)=n-k(\chi(\mathbf{M})$ обозначает ранг матрицы $\mathbf{M})$ для 
$\gamma \in\left(\mathfrak{F}, \mathfrak{B S}\right.$ область в $E^{k}$, то краевая задача $(\lambda)$ имеет для $\gamma \in(\mathfrak{S}$ точно $k$ линейно независимых решений (и наоборот). Тогда также сопряженная краевая задача $\left(\lambda^{*}\right)$

$$
\dot{\boldsymbol{w}}^{\prime}=-\boldsymbol{w}^{\prime} \frac{\mathbf{D} \boldsymbol{f}}{\mathbf{D x}}(t, \tilde{\boldsymbol{\eta}}(t)), \quad \boldsymbol{w}^{\prime}(a) \mathbf{P}^{\prime}(\gamma)+\boldsymbol{w}^{\prime}(b) \mathbf{Q}^{\prime}(\gamma)=0
$$

(' обозначает транспонирование), где $n \times n$ матрицы $\mathbf{P}(\gamma), \mathbf{Q}(\gamma)$ для $\gamma \in$ (5) удовлетворяют соотношениям

$$
-\frac{\mathbf{D u}}{\mathbf{D} \mathbf{x}_{\boldsymbol{a}}}\left(\tilde{\boldsymbol{\eta}}_{a}, \tilde{\boldsymbol{\eta}}_{b}\right) \boldsymbol{P}^{\prime}(\gamma)+\frac{\mathbf{D u}}{\mathbf{D} \boldsymbol{x}_{b}}\left(\tilde{\boldsymbol{\eta}}_{a}, \tilde{\boldsymbol{\eta}}_{b}\right) \mathbf{Q}^{\prime}(\gamma) \equiv 0, \quad \chi(\boldsymbol{P}(\gamma): \mathbf{Q}(\gamma))=n,
$$

имеет точно $k$ линейно независимых решений.

Можно доказать следующие две теоремы:

Теорема 3.1. Пусть краевая задача $\left(v_{0}\right)$ имеет изолированное решение $\boldsymbol{\eta}\left(t, \boldsymbol{c}_{0}^{*}\right)$ и пусть краевая задача $(\lambda)$ для $\tilde{\boldsymbol{\eta}}(t)=\boldsymbol{\eta}\left(t, \boldsymbol{c}_{0}^{*}\right)$ имеет только тривиальное решение. Если имеют место еще некоторые дальнейшие предположения (например, гладкости), то краевая задача (v) для достаточно малых в имеет одно однозначно определенное решение $\xi\left(t, c^{*}(\varepsilon), \varepsilon\right)$ с начальным значением $\xi\left(a, c^{*}(\varepsilon), \varepsilon\right)=$ $=\boldsymbol{c}^{*}(\varepsilon)$ такое, что $\xi\left(t, \boldsymbol{c}^{*}(0), 0\right)=\boldsymbol{\eta}\left(t, \boldsymbol{c}_{0}^{*}\right), \boldsymbol{c}^{*}(0)=\boldsymbol{c}_{0}^{*}$.

Теорема 3.2. Пусть задача $\left(v_{0}\right)$ имеет $k$-параметрическое семейство решений $\boldsymbol{\eta}\left(t, \varrho_{0}(\gamma)\right), \boldsymbol{\gamma} \in \mathfrak{G}, \mathfrak{G}$ - открытое множсество в $E^{k}$. Пусть задача $(\lambda)$ имеет для $\gamma \in \mathfrak{G}_{1}, \mathfrak{G H}_{1}$ - открытое множество в $\mathfrak{G}$, точно $k$ линейно независимых решений. Из этого вытекает, что

$$
\chi\left(\frac{\mathbf{D u}}{\mathbf{D c}}\left(\tilde{\boldsymbol{\eta}}_{a}, \tilde{\boldsymbol{\eta}}_{b}\right)\right)=n-k .
$$

Пусть, в частности,

$$
\frac{\mathbf{D} \boldsymbol{u}_{\mathscr{s}}}{\mathbf{D c}}\left(\tilde{\boldsymbol{\eta}}_{a}, \tilde{\boldsymbol{\eta}}_{b}\right)-\mathbf{T}(\gamma) \frac{\mathbf{D} \boldsymbol{u}_{\overline{\mathscr{s}}}}{\mathbf{D c}}\left(\tilde{\boldsymbol{\eta}}_{a}, \tilde{\boldsymbol{\eta}}_{b}\right) \equiv 0,
$$

где $\boldsymbol{T}(\gamma)-$ матрича типа $k \times(n-k), \mathscr{S}-$ упорядоченная система $k$ индексов из $\{1,2, \ldots, n\}=\mathscr{N}, \overline{\mathscr{S}}-$ дополнение $\mathscr{S}$ относительно $\mathscr{N}$.

Можно показать, что существует матрица $\Psi(t, \gamma)$ типа $k \times n$ такая, что ее строки являются решениями сопряженной задачи $\left(\lambda^{*}\right)$ и что она удовлетворяет краевому условию

$$
\boldsymbol{\Psi}(b, \gamma)=\frac{\mathbf{D} \boldsymbol{u}_{\mathscr{S}}}{\mathbf{D} \boldsymbol{x}_{b}}\left(\tilde{\boldsymbol{\eta}}_{a}, \tilde{\boldsymbol{\eta}}_{b}\right)-\boldsymbol{T}(\gamma) \frac{\mathbf{D} \boldsymbol{u}_{\overline{\mathscr{Y}}}}{\mathbf{D} \boldsymbol{x}_{b}}\left(\tilde{\boldsymbol{\eta}}_{a}, \tilde{\boldsymbol{\eta}}_{b}\right) .
$$

Пусть система уравнений

$$
\int_{a}^{b} \boldsymbol{\Psi}\left(s, \gamma_{0}\right) \mathbf{g}\left(s, \boldsymbol{\eta}\left(s, \varrho_{0}\left(\gamma_{0}\right)\right), 0\right) \mathrm{d} s+\boldsymbol{v}_{0 \mathscr{S}}-\boldsymbol{T}\left(\gamma_{0}\right) \boldsymbol{v}_{0 \overline{\mathscr{S}}}=0
$$


(где $\left.\boldsymbol{v}_{0}=\boldsymbol{v}\left(\boldsymbol{\eta}\left(a, \varrho_{0}\left(\gamma_{0}\right)\right), \boldsymbol{\eta}\left(b, \varrho_{0}\left(\gamma_{0}\right)\right), 0\right)\right)$ имеет действительное решение $\gamma_{0}=$ $=\gamma_{0}^{*} \in B_{1}$.

Пусть якобиян системы (3.21) относительно $\gamma_{0}$ в точке $\gamma_{0}=\gamma_{0}^{*}$ отличен от нуля.

Тогда при некоторых предположсениях гладкости (и некоторых дальнейших менее существенных) для достаточно малых в существует одно однозначно определенное рещение $\xi\left(t, \boldsymbol{c}^{*}(\varepsilon), \varepsilon\right), \xi\left(a, \boldsymbol{c}^{*}(\varepsilon), \varepsilon\right)=\boldsymbol{c}^{*}(\varepsilon)$ задачи $(v)$ такое, что $\xi\left(t, \varrho_{0}\left(\gamma_{0}^{*}\right), 0\right)=\boldsymbol{\eta}\left(t, \varrho_{0}\left(\gamma_{0}^{*}\right)\right), \boldsymbol{c}^{*}(0)=\varrho_{0}\left(\gamma_{0}^{*}\right)$.

Наконец, в четвертом параграфе исследуются краевые задачи, для которых система дифференциальных уравнений автономна, т. е.

$$
\dot{\boldsymbol{x}}=\boldsymbol{f}(\mathbf{x})+\varepsilon \boldsymbol{g}(\mathbf{x}, \varepsilon) \text {. }
$$

Краевую задачу $(4.1),(3.2)$ обозначим через $(\alpha)$ и соответствующую укороченную задачу через $\left(\alpha_{0}\right)$.

Вводится следующее основное понятие: Скажем, что краевая задача $(\alpha)$ имеет свойство $(\mathscr{P})$, если имеет место: Если $\varphi^{*}(t)-$ решение краевой задачи $(\alpha)$ на определенном интервале $\langle a, b\rangle$, то также

$$
\boldsymbol{w}\left(\varphi^{*}(a+\delta), \varphi^{*}(b+\delta), \varepsilon\right)=0
$$

лдя любого $\delta$, для которого функция $\varphi^{*}(t+\delta)$ имеет значение.

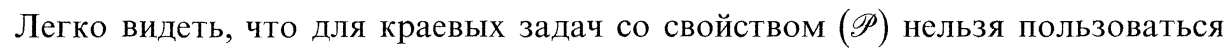
теоремами третьего параграфа, так как решения этих задач образуют по меньшей мере однопараметрические системы. Поэтому мы должны одну из компонент начального вектора искомого решения фиксировать, и показывается необходимым вместо этой переменной выбрать длину интервала $\langle a, b(\varepsilon)\rangle$, на котором ищется решение,как новую неизвестную.

(Очевидно, что краевые задачи с периодическими краевыми условиями имеют свойство $(\mathscr{P})$. Эти задачи занимают знаменательное положение среди краевых задач со свойством $(\mathscr{P})$.)

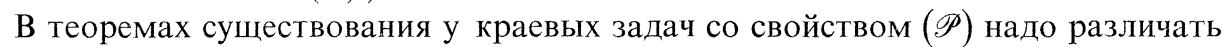
случай, когда укороченная краевая задача $\left(\alpha_{0}\right)$ имеет $k$-параметрическое семейство решений на интервале $\langle a, b\rangle$ постоянной длины (теоремы 4.1 и 4.2), и случай, когда задача $\left(\alpha_{0}\right)$ имеет семейство решений, зависящее от $k$ параметров $\gamma=\left(\gamma_{1}, \gamma_{2}, \ldots, \gamma_{k}\right)$ и сушествующее на интервале $\langle a, b(\gamma)\rangle$.

В обоих случаях получим теорему, аналогичную теоремам 3.2 и 3.1 . 